\title{
Maverick: A Stand-Alone CAD Flow for Partially Reconfigurable FPGA Modules
}

Dallon Godfrey Glick

Brigham Young University

Follow this and additional works at: https://scholarsarchive.byu.edu/etd

Part of the Engineering Commons

\section{BYU ScholarsArchive Citation}

Glick, Dallon Godfrey, "Maverick: A Stand-Alone CAD Flow for Partially Reconfigurable FPGA Modules" (2019). Theses and Dissertations. 7746.

https://scholarsarchive.byu.edu/etd/7746

This Thesis is brought to you for free and open access by BYU ScholarsArchive. It has been accepted for inclusion in Theses and Dissertations by an authorized administrator of BYU ScholarsArchive. For more information, please contact ellen_amatangelo@byu.edu. 
Maverick: A Stand-Alone CAD Flow for Partially

Reconfigurable FPGA Modules

\title{
Dallon Godfrey Glick
}

\begin{abstract}
A thesis submitted to the faculty of
Brigham Young University

Master of Science

Brent E. Nelson, Chair

Michael J. Wirthlin

Jeffrey B. Goeders
\end{abstract}

in partial fulfillment of the requirements for the degree of

Department of Electrical and Computer Engineering

Brigham Young University

Copyright (c) 2019 Dallon Godfrey Glick

All Rights Reserved 


\author{
ABSTRACT \\ Maverick: A Stand-Alone CAD Flow for Partially \\ Reconfigurable FPGA Modules \\ Dallon Godfrey Glick \\ Department of Electrical and Computer Engineering, BYU \\ Master of Science
}

Circuit designs for field-programmable gate arrays (FPGAs) are typically compiled by FPGA vendor tools, such as Xilinx's Vivado Design Suite. In recent years, partial reconfiguration (PR) has emerged as a popular technique that allows portions of an FPGA to be dynamically reconfigured after the complete device has been configured with an initial bitstream. However, the nature of current FPGA vendor tools limits further innovation and possible usage models of PR.

This thesis presents Maverick, an open-source proof-of-concept computer-aided design (CAD) flow for generating reconfigurable modules (RMs) which target PR regions in FPGA designs. Maverick builds upon existing open source tools (Yosys [1], RapidSmith2 [2], and Project X-Ray [3]) to form an end-to-end compilation flow. After an initial static design and PR region are created with Xilinx's Vivado PR flow, Maverick can then compile and configure RMs onto that PR region-without the use of vendor tools. In addition, this work enables users to import and export RMs between Vivado and RapidSmith2.

Furthermore, this thesis demonstrates Maverick compiling RMs on both a desktop computer and on the embedded PYNQ-Z1 board, which contains a Zynq 7020 system on chip (SoC). Maverick runs on the ARM processor embedded within the processing system (PS) of the Zynq device, generating partial bitstreams which can then be configured onto a PR region within the programmable logic (PL) fabric of the same Zynq device. This unique case, not possible with current vendor tools like Vivado, demonstrates the feasibility of a single-chip embedded system which can both compile HDL designs to bitstreams and then configure them onto its own programmable fabric.

Keywords: FPGA, Partial Reconfiguration, EDA, CAD, Xilinx, Open-Source 


\section{ACKNOWLEDGMENTS}

I am thankful toward several people that have encouraged or helped me to complete this thesis and master's degree. First and foremost, I would like to thank my parents Benjamin and June Glick, who have been both supportive and encouraging to me throughout my life. In particular,

they have instilled in me the desire to learn and have also taught me diligence, which has greatly helped me through my years in schooling.

I would also like to thank my friends and colleagues in the Configurable Computing Lab. Some have been near constants throughout my studies at Brigham Young University. I am thankful for the opportunity I had to work with so many of them on various school and lab projects over the years. I owe particular thanks to Travis Haroldsen and Thomas Townsend, whose prior work on RapidSmith2, RSVPack, and RSVPlace was instrumental to this work; both also took the time to help me along this work. In addition, I thank Jesse Grigg and Reilly McKendrick for their help in making this thesis possible.

I would also like to thank my graduate committee, consisting of Dr. Brent Nelson, Dr. Michael Wirthlin, and Dr. Jeffrey Goeders. Dr. Wirthlin introduced me to the Configurable Computing Lab in the senior year of my undergraduate career and was heavily involved throughout this work. Dr. Goeders also provided helpful guidance on FPGA CAD tools and algorithms inside and outside of class. I especially need to thank my advisor, Dr. Brent Nelson, without whom this thesis would not have been possible. I greatly appreciate not only his expertise on FPGAs but also his exceptional patience and kindness, which has helped me complete this work.

Finally, I must also acknowledge the major source of funding that supported the research described in this thesis. This work was supported in part by the I/UCRC Program of the National Science Foundation within the NSF center for Space, High-performance, and Resilient Computing (SHREC) under Grant No. 1738550. The opinions, findings, and conclusions or recommendations expressed are those of the author(s) and do not necessarily reflect the views of the National Science Foundation. 


\section{TABLE OF CONTENTS}

TABLE OF CONTENTS $\ldots \ldots \ldots \ldots \ldots \ldots \ldots \ldots \ldots$ iv

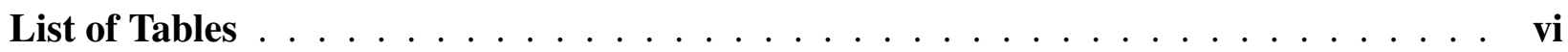



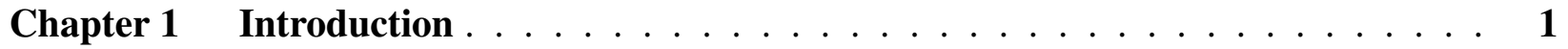

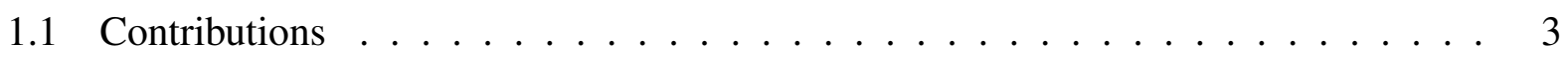

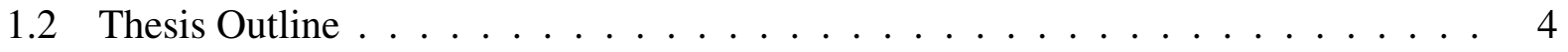

Chapter 2 FPGAs and Partial Reconfiguration $\ldots \ldots \ldots \ldots \ldots \ldots$

2.1 FPGA Architecture . . . . . . . . . . . . . . . . . 5

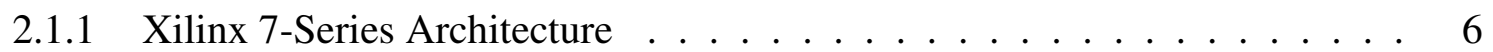



2.1 .3 Sites . . . . . . . . . . . . . . . . . . 8

2.1 .4 Tiles . . . . . . . . . . . . . . . . . . . . . . . . . . . . 9

2.1.5 Clock Region . . . . . . . . . . . . . . . . . . . . . . . . 11

2.2 FPGA CAD Flow . . . . . . . . . . . . . . . . . . . 11

2.2 .1 Vivado CAD Flow . . . . . . . . . . . . . . . . . . . . . 12

2.3 Partial Reconfiguration . . . . . . . . . . . . . . . . . . . . . . 14

2.3.1 Xilinx Partial Reconfiguration Flow . . . . . . . . . . . . . . 15

Chapter 3 Third-Party CAD Tools and Related Work . . . . . . . . . . . . . . . 22

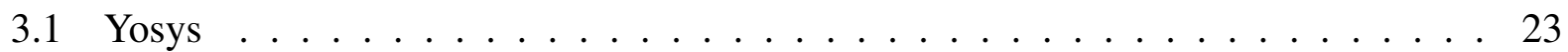

3.2 RapidSmith2 . . . . . . . . . . . . . . . . . . . . . . . . . 24

3.3 RapidSmith2's Interface with Vivado . . . . . . . . . . . . . . . . . . 25

3.3 .1 Tincr . . . . . . . . . . . . . . . . . . . 25

3.3.2 Vivado Design Interface . . . . . . . . . . . . . . . . . . 25

3.4 Project X-Ray . . . . . . . . . . . . . . . . . . . . . . . . 27

Chapter 4 RapidSmith2: Partial Reconfiguration Support . . . . . . . . . . . . . . . 29

4.1 PR in RapidSmith2 . . . . . . . . . . . . . . . . . . . . . . . . . . . . . . . . . . 29

4.1.1 Partial Device Representation . . . . . . . . . . . . . . . . . . . . 29

4.1.2 Partition Pins in RapidSmith2 _. . . . . . . . . . . . . . . . . . . 31

4.2 Reconfigurable Module (RM) RapidSmith Checkpoints (RSCPs) . . . . . . . . . 31

$4.2 .1 \quad$ RM RSCP Format . . . . . . . . . . . . . . . . . . . . . . . . . . 31

4.2 .2 Exporting RM RSCPs from Vivado . . . . . . . . . . . . . . . . . . . . 34

4.2 .3 Importing RM RSCPs into RapidSmith2 . . . . . . . . . . . . . . . . . . 37

4.3 Reconfigurable Module (RM) Tincr Checkpoints (TCPs) . . . . . . . . . . . 40

4.3 .1 RM TCP Format . . . . . . . . . . . . . . . . . . . . . . . . . 41

4.3.2 Exporting RM TCPs from RapidSmith2 . . . . . . . . . . . . . . . . . . . 42

4.3 .3 Importing RM TCPs into Vivado . . . . . . . . . . . . . . . 43 


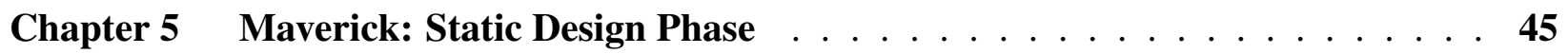

5.1 Overview of Maverick . . . . . . . . . . . . . . . . . 45

5.2 Static Design Creation: Vivado . . . . . . . . . . . . . . . . . . . . 46

5.3 Partial Device Generation: RapidSmith2 . . . . . . . . . . . . . . . . . . . 47

5.4 Bitstream Database Generation: Project X-Ray . . . . . . . . . . . . . . . 47

Chapter 6 Maverick: Reconfigurable Module Phase _ . . . . . . . . . . . . . 49

6.1 Synthesis and Tech-Mapping: Yosys . . . . . . . . . . . . . . . . 50

6.2 RapidSmith2 Design Import $\ldots \ldots \ldots$. . . . . . . . . . . . . . 51

6.3 Packing: RSVPack . . . . . . . . . . . . . . . . . . . 52

6.4 Placement: RSVPlace . . . . . . . . . . . . . . . . . . . . . . . 53

6.5 Routing: RSVRoute . . . . . . . . . . . . . . . . . . . . . . . 53

6.5 .1 Preparing Nets for Routing . . . . . . . . . . . . . . . . . . . 54

6.5 .2 PathFinder . . . . . . . . . . . . . . . . . . . 57

6.5 .3 Limitations . . . . . . . . . . . . . . . . . . . 63

6.5.4 Partial Reconfiguration (PR) Features . . . . . . . . . . . . . . . . . 64

6.6 FASM Generation: RapidSmith2 . . . . . . . . . . . . . . . . . . . . . . 64

6.6 .1 Logic BEL Instructions . . . . . . . . . . . . . . . . . . . . 65

6.6 .2 Site-Wide Instructions . . . . . . . . . . . . . . . . . . . . . . . . . . . . . . 69

6.6 .3 Inter-Site Routing Instructions . . . . . . . . . . . . . . . . . . 70

6.6 .4 Static Design Instructions . . . . . . . . . . . . . . . . . . . 71

6.7 Bitstream Generation: Project X-Ray . . . . . . . . . . . . . . . . . 71

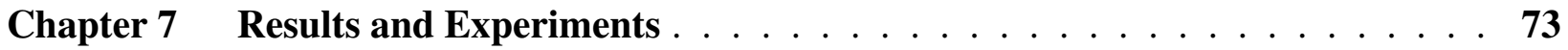

$7.1 \quad$ RSVRoute Results . . . . . . . . . . . . . . . . . . . . . . . . . . . . . 73

7.2 Maverick Results . . . . . . . . . . . . . . . . . . . . . . . . . 77

$7.2 .1 \quad$ Static Design Phase Results . . . . . . . . . . . . . . . . . . . . . . 78

7.2.2 Reconfigurable Module (RM) Phase Results . . . . . . . . . . . . . . . . . 81

7.3 Maverick on the PYNQ-Z1 . . . . . . . . . . . . . . . . . . . . . . 84

$7.3 .1 \quad$ RM Phase Results . . . . . . . . . . . . . . . . . . . . . . . . . . 85

7.3 .2 Experimental Demo . . . . . . . . . . . . . . . . . 88

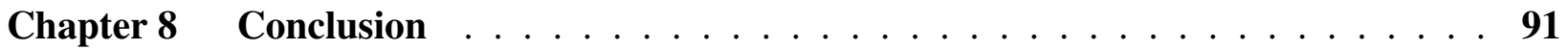

8.1 Future Work . . . . . . . . . . . . . . . . . . . . . . 92

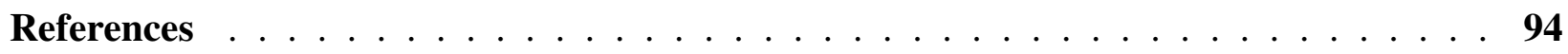




\section{LIST OF TABLES}

6.1 Truth Table for Equation 6.6. Rows with $\mathrm{O} 6=0$ are Omitted. . . . . . . . . . . 65

7.1 RSVRoute Benchmarks . . . . . . . . . . . . . . . . . . . . . . . 74

7.2 Comparison of Routing Run-times (in Seconds) . . . . . . . . . . . . . . . . . 75

7.3 Routing Wire-length Comparison (in Tiles Traversed) . . . . . . . . . . . . . . . 76

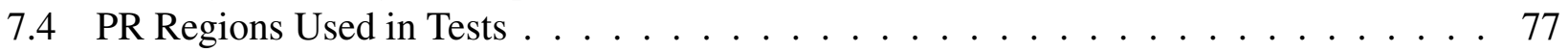

7.5 PR Benchmark Designs . . . . . . . . . . . . . . . . . . . . . 78

7.6 Static Design Resource Usage . . . . . . . . . . . . . . . . . . . . . . . . . . . . . . . . . . . . . . . . . .

7.7 VDI Static Design Export Times (in Seconds) . . . . . . . . . . . . . . . . . . 80

7.8 Quality of the Implemented RM Benchmarks . . . . . . . . . . . . . . . 81

7.9 RM Phase Run-times on a Desktop PC (in Seconds) . . . . . . . . . . . . . . . . . . . 83

7.10 RM Phase Run-times on the PYNQ-Z1 (in Seconds) . . . . . . . . . . . . . . . . . 86

7.11 RM Phase Peak Memory Usage on the PYNQ-Z1 (in MB) . . . . . . . . . . . . . 87 


\section{LIST OF FIGURES}

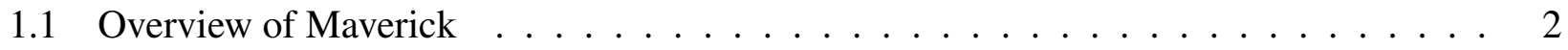

2.1 Generalized Island-Style FPGA Architecture $\ldots \ldots \ldots \ldots \ldots \ldots$

2.2 Xilinx FPGA Hierarchy . . . . . . . . . . . . . . . . . . . . . 6

2.3 BELs in Vivado . . . . . . . . . . . . . . . . . . . . . 7

2.4 Logic BEL Route-throughs . . . . . . . . . . . . . . . . . . . . 7

2.5 A 7-Series SLICEL Site . . . . . . . . . . . . . . . . . . . . 8

2.6 A SLICEL Being Used as a Site Route-Through . . . . . . . . . . . . . . . . . 9

2.7 An Interconnect Tile . . . . . . . . . . . . . . . . . . . . . . . . . . . . . . . . 10

2.8 A Node - Reproduced from [4] . . . . . . . . . . . . . . . . . . . . . . 11

2.9 Correspondence Between the Traditional and Vivado CAD Flows . . . . . . . . . . . 12

2.10 Organization of Standard Xilinx Bitstreams . . . . . . . . . . . . . . . . 14

2.11 Module-Based PR Configuration Styles _ . . . . . . . . . . . . . . . . . . 15

2.12 Vivado's Single-Island PR Style . . . . . . . . . . . . . . . . . . . . . . . 16

2.13 A Partial Route to a Partition Pin . . . . . . . . . . . . . . . . . . . . 18

2.14 Organization of Partial Bitstreams f . . . . . . . . . . . . . 20

3.1 Yosys Phases. Taken from $[5] \ldots \ldots \ldots \ldots . \ldots . \ldots . \ldots \ldots$

3.2 RapidSmith2 Tool Flow Using Tincr. Adapted from [4]. . . . . . . . . . . . . . . . . 26

4.1 Partial Device Representation in Tiles _ . . . . . . . . . . . . . . . . . . . . . . 29

4.2 Visualization of RapidSmith2 Routing Data Structures . . . . . . . . . . . . . . 40

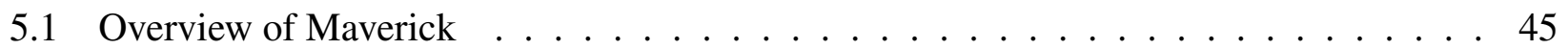

6.1 Detailed Overview of Maverick's RM Phase . . . . . . . . . . . . . . . . . . . . . 49

6.2 A CARRY4 BEL in Vivado with Internal MUXCY and XORCY BELs . . . . . . . . . 52

6.3 The Start Tree of an Inter-Site Route . . . . . . . . . . . . . . . . . . . . . . . . 55

6.4 A Vivado VCC Net with Two Tie-Off Sources . . . . . . . . . . . . . . . . . . 56

6.5 A LUT Static Source Used to Route a VCC Sink . . . . . . . . . . . . . . . . . . 60

6.6 Two Methods of Routing a Multi-Sink Net. Taken from [6]. . . . . . . . . . . . . . . 61

6.7 A Routed Global Clock Buffer Net and a Routed Local Clock Net . . . . . . . . . . . 62

6.8 Organization of Partial Bitstreams Produced by xc7PartialPatch . . . . . . . . . . . 72

7.1 RM Phase Run-Times on a Desktop PC (in Seconds) . . . . . . . . . . . . . . . . . . 82

7.2 RM Phase Run-Times on the PYNQ-Z1 (in Seconds) . . . . . . . . . . . . . . . . 85

7.3 Jupyter Notebook for Maverick's RM Phase . . . . . . . . . . . . . . . . . . . . . . . 89

7.4 The PYNQ-Z1 Board Configured with a Stopwatch Partial Bitstream . . . . . . . . . . 89 


\section{CHAPTER 1. INTRODUCTION}

Vendors of commercial field-programmable gate arrays (FPGAs) provide computer-aided design (CAD) tools to support the design of circuits that target their FPGAs. Such tools include Xilinx's Vivado Design Suite, Intel's Quartus Prime, and Microchip's Libero SoC Design Suite. These tools embody incredibly complex algorithms to generate high-quality circuits with limited computing time. All vendor tools provide the ability to generate bitstreams that can program a full FPGA and some also provide a level of support for partial reconfiguration (PR), a technique whereby portions of a FPGA can be dynamically reconfigured after the complete FPGA has been initially configured. While the vendor tools satisfy the needs of most users, they do, however, come with some limitations.

Firstly, the vendor tools are all closed-source and allow limited customization of the CAD flow. This makes the jobs of FPGA researchers interested in new FPGA CAD algorithms, security, and novel use-cases more difficult. As a result, FPGA researchers have typically had to develop and use alternative tools. Verilog-to-Routing (VTR) [7] is one example of an alternative CAD suite and has been commonly used for experimentation on hypothetical FPGA architectures. Tools which can target Xilinx FPGAs have also been developed, such as RapidSmith [8], Torc [9], RapidSmith2, [2] and RapidWright [10]. However, these tools have traditionally returned to the vendor tools at least to generate final bitstreams.

Another limitation of the vendor tools is the system requirements to run them. FPGA vendor CAD tools are meant be run on desktop workstations, with performance suffering on computers with less powerful resources. For instance, Xilinx's Vivado can only be run on x86 or x86-64 machines and requires a minimum of 48 GB of RAM to create bitstreams for the largest devices [11] [12]. Additionally, a considerable amount of disk space is often required by the vendor tools, with a full Vivado installation requiring over 52 GB of disk space. These requirements limit the types of platforms that can compile FPGA bitstreams. 
Finally, we believe that the PR support provided in vendor tools does not allow the full potential of PR to be realized. Compiling a partial bitstream for a PR region in Vivado requires the availability of the original PR project files, including the full Vivado design, no matter how small the reconfigurable module (RM). Additionally, a Vivado installation is always required, tethering the creation of partial bitstreams to desktop computers that can run Vivado.

This work set out to answer a number of questions posed by these limitations. The first goal of this work was to investigate the possibility of creating a Xilinx FPGA CAD flow that did not need to rely on the Xilinx tools to fully compile designs. The second goal of this work was to add support for PR to RapidSmith2, allowing PR designs to be exchanged between RapidSmith2 and Vivado. Finally, the last and main goal of this work was to enable the compilation of partial bitstreams on an embedded device, such as a Zynq system on a chip (SoC) containing both an FPGA and a processor.

These goals were met with the main result of this work, Maverick. Maverick is a full flow for compiling Xilinx 7-Series RMs from Verilog to partial bitstreams. Maverick separates initial static design creation from subsequent RM compilation. Figure 1.1 gives an overview of Maverick's two phases. In the first phase, the static design phase, a static design is created using Vivado's PR flow. Data about this design and the PR region is then generated using Vivado, RapidSmith2, and Project X-Ray [3]. Using this generated data, the RM phase of Maverick can then compile RMs from Verilog to partial bitstreams. The compilation of the RMs is performed using Yosys [1], RapidSmith2, and Project X-Ray.

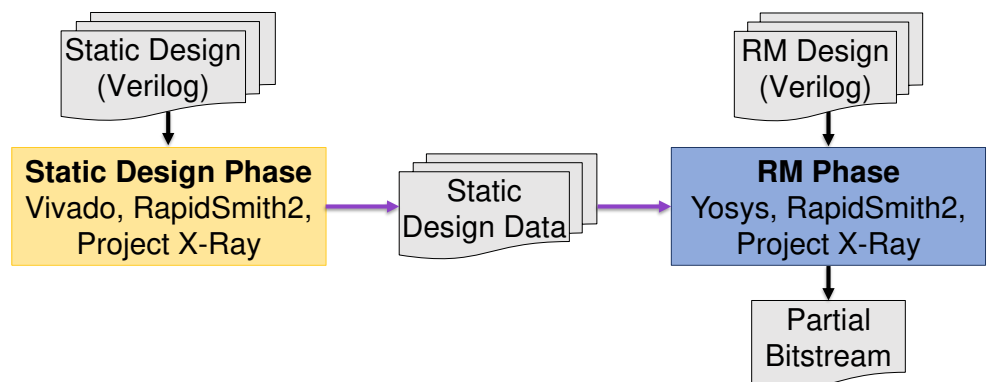

Figure 1.1: Overview of Maverick

Maverick shows the feasibility of 3rd-party flows for Xilinx devices and opens up new usage models for PR. Because the RM phase is independent of Vivado, it can run on a variety of 
systems, including embedded systems. This could enable the creation of fully autonomous systems which are untethered from other compute resources. In this model, adaptation algorithms running on the autonomous platform could create new HDL design code for an RM (or modify existing RM HDL code) which would then be compiled by Maverick and configured onto the platform's FPGA fabric. Such autonomous systems could use this model to provide domain specific functionality or to improve fault tolerance. Another potential usage model enabled by Maverick is a customized system tailored for education, which could mix instructional materials, sample designs, HDL code, and CAD tools into a lightweight standalone hardware platform.

\subsection{Contributions}

The main contributions of this thesis are as follows:

- It provides Maverick, a non-vendor flow for PR regions of Xilinx 7-Series FPGAs. The components of Maverick are all released as open-source at https://github.com/byuccl/maverick.

- It provides a Jupyter Lab Interface for running Maverick on Digilent's PYNQ-Z1 board.

- It adds PR support to RapidSmith2 and Tincr, a Vivado Tcl Plugin [13]. This allows designs targeting PR regions to be manipulated within the RapidSmith2 framework and for the designs to be exchanged between Vivado and RapidSmith2.

- It provides RSVRoute, a router built within RapidSmith2. Importantly, this router supports full-device designs as well as RM designs targeting PR regions. When combined with the existing RSVPack and RSVPlace tools, this provides RapidSmith2 with a full physical design flow for Xilinx 7-Series FPGAs, making Maverick possible. The quality and performance of RSVRoute are also analyzed through the results of RSVRoute routing a set of benchmark designs.

- It adds support for generating partial bitstreams, for documented regions of the Xilinx xc7z020 FPGA, to the Project X-Ray tools.

- It presents the results of Maverick executing on a desktop computer and on the PYNQ-Z1 board, showing the feasibility of executing stand-alone CAD flows for commercial FPGAs 
on embedded systems. The resulting circuit quality of a set of benchmarks and the performance of Maverick are both presented.

\subsection{Thesis Outline}

This thesis is outlined as follows. Chapter 2 first provides necessary background information on FPGAs and partial reconfiguration. Chapter 3 then reviews related third-party CAD tools and related work. The CAD tools that make up Maverick are given particular attention. Chapter 4 describes the additions that were made to RapidSmith2 and the Vivado Design Interface (VDI) to support PR and Maverick. Chapter 5 then describes the static design phase of Maverick, in which a static design is created with Vivado to enable the subsequent compilation of partial bitstreams. Chapter 6 next describes the RM phase of Maverick, in which Maverick compiles RM designs targeting PR regions to partial bitstreams. Chapter 7 presents the experiments and results of this work. This includes results on RSVRoute, Maverick's static phase, and Maverick's RM phase. An experiment in which Maverick was integrated with the PYNQ system on the PYNQ-Z1 board is also presented. This experiment included compiling a number of designs with Maverick on the PYNQ-Z1, programming them onto the same board, and then verifying their correct functionality. Chapter 8 finally concludes this thesis and discusses the possibilities for future work. 


\section{CHAPTER 2. FPGAS AND PARTIAL RECONFIGURATION}

This chapter reviews essential background information on FPGAs and partial reconfiguration (PR). First, this chapter describes the architecture of FPGAs, focusing on the Xilinx 7-Series architecture. Then, the traditional FPGA CAD flow is described, again focusing on the specific CAD flow used by Xilinx. Lastly, the concept of PR is explained, with Xilinx's specific approach to PR being highlighted.

\subsection{FPGA Architecture}

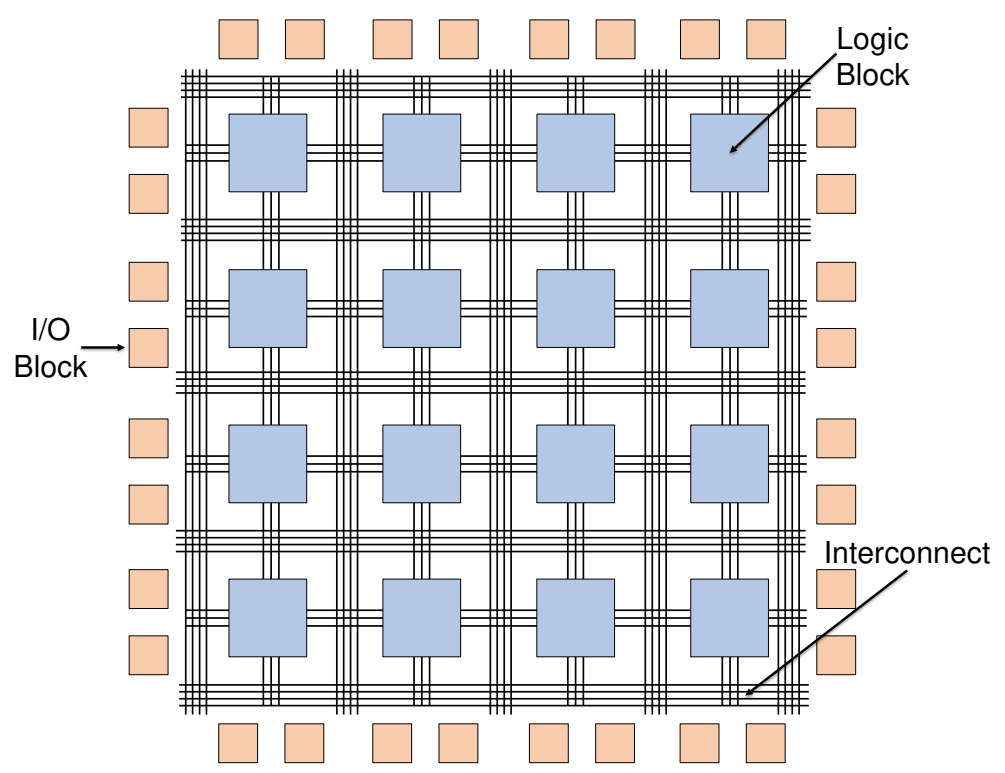

Figure 2.1: Generalized Island-Style FPGA Architecture

A field-programmable gate array (FPGA) is an integrated circuit that can be reprogrammed in the field to implement different digital circuits. The architecture of an FPGA is crucial to providing this flexibility. The most common type of FPGA is made up of a two-dimensional grid of configurable logic blocks (CLBs), as shown in Figure 2.1; this is sometimes referred to as an 
island-style FPGA. These CLBs are the basic building blocks of the FPGA and contain at least a look-up table (LUT) and a flip-flop (FF). Modern FPGAs also contain hard blocks with fixed functionality, such as multiplier blocks and memory blocks. A flexible routing interconnect connects all of these blocks to each other and to external input/output (I/O) blocks.

\subsubsection{Xilinx 7-Series Architecture}

Xilinx 7-Series FPGA devices are structured into four main levels of hierarchy. From bottom to top, these levels are Basic Elements of Logic (BELs), sites, tiles, and clock regions (also known as fabric sub-regions). This hierarchy can be seen in Figure 2.2.

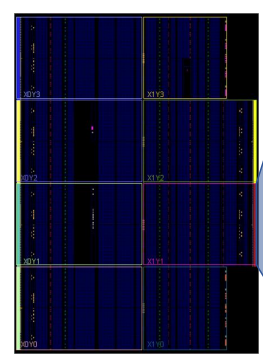

Device

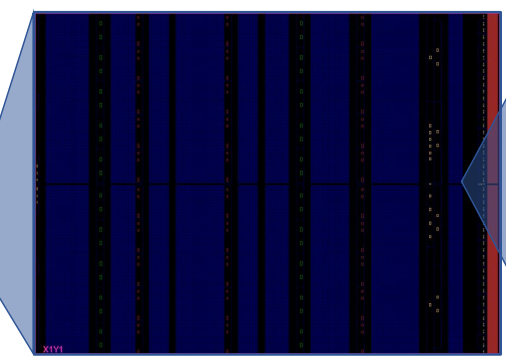

Clock Region

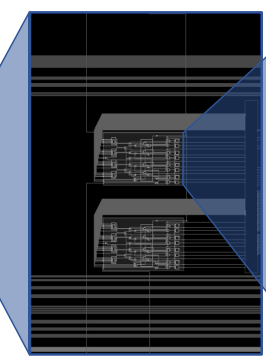

Tile



Site

Figure 2.2: Xilinx FPGA Hierarchy

\subsubsection{BELS}

The lowest level of hierarchy is the BEL, of which there are two kinds: logic BELs and routing BELs. Logic BELs are configured by the assignment of logical cell primitives contained in a logical netlist, as Section 2.2 explains. Usually, a single logical cell maps to a single logic BEL. However, macro cells, which contain one or more internal cells, are sometimes used. Macro cells assign their internal cells to closely located logic BELS.

The most common logic BELs are LUT and FF BELs, with LUT BELs implementing logic equations and FF BELs being used for storage. The LUTs used in the Xilinx 7-Series architecture are fracturable 6-input LUTs, meaning each can be used as two 5-input LUTs sharing 5 common inputs. The left side of Figure 2.3 shows an example of a LUT BEL, as seen in Vivado. 




Logic BEL
BEL Pins



Routing BEL

Figure 2.3: BELs in Vivado

Routing BELs include routing muxes and polarity muxes (also known as polarity selectors). The routing muxes are used to route different signals between BELs and are not typically configured by logical cell primitives. The right side of Figure 2.3 shows an example of a routing mux in Vivado. Polarity muxes can be used to optionally invert a signal and can be configured by certain properties in a logical netlist.

Both logic and routing BELs have input and output pins, as highlighted in green in Figure 2.3. A BEL's input pin can be configured to connect to one of its output pins. In the case of routing BELS, these configurable connections are referred to as site programmable interconnect points (PIPs). A routing BEL is useless if none of its site PIPs is configured.

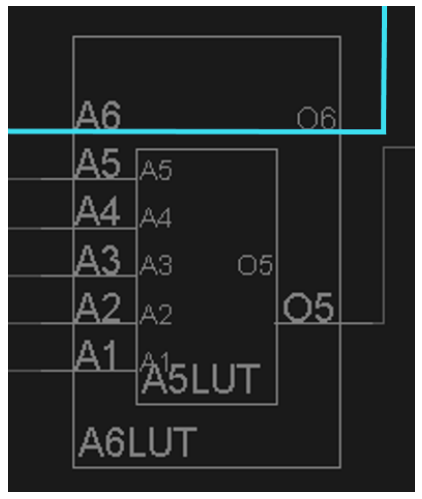

(a) A LUT Route-Through

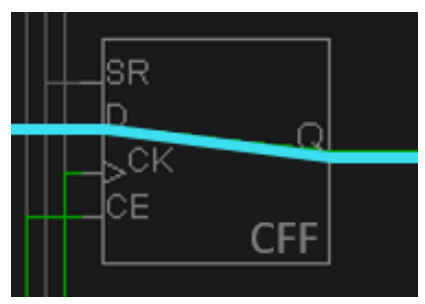

(b) A FF Route-through

Figure 2.4: Logic BEL Route-throughs 
A logic BELs' pins are normally not configured to connect to each other in this way. However, if a logic BEL is unoccupied by a logical cell primitive, it may be configured as a routethrough, as shown in Figure 2.4. Route-throughs are sometimes necessary in order to fully route a design. Both LUT and FF BELs may be configured as route-throughs, with FF route-throughs also being referred to as permanent latches. A LUT BEL can also be configured as a static signal (VCC or GND) source. Similar to route-throughs, these are normally only used when necessary for the routing of a design.

\subsubsection{Sites}

The site is the next level of hierarchy. As can be seen in Figure 2.5, a site consists of three main components: BELs, site wires, and site pins. The site wires connect BELs within a site together, and site pins connect the site to wires outside of the site. The Xilinx 7-Series architecture includes a variety of different types of sites.

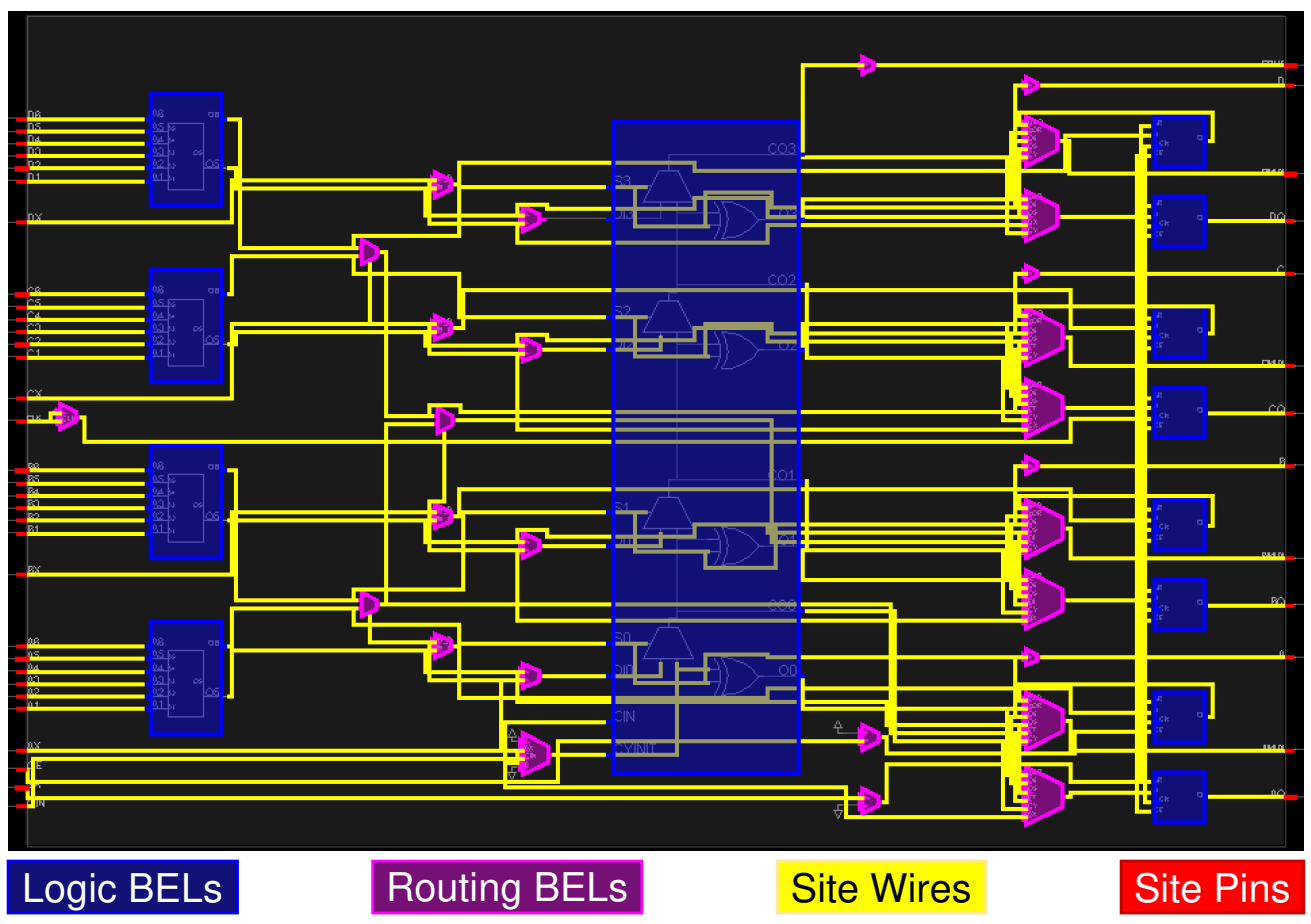

Figure 2.5: A 7-Series SLICEL Site 
The most common type of sites are the SLICEL and SLICEM sites, collectively referred to as slices. A SLICEL, shown in Figure 2.5, contains LUT BELs, FF BELs, a carry-chain BEL, routing BELS, site wires, and site pins. A SLICEM is similar to a SLICEL but contains additional routing for the use of LUT RAMs and shift registers (SRLs).

If a site does not contain any logic BELs that have been configured with logical cell primitives, it can be instead be used as a routing resource if necessary [14]. When a site is used in this way, it is called a site route-through. In Vivado, a site route-through is viewed as a PIP from one of a site's pins to one of its output pins (most site route-throughs are input pin to output pin route-throughs, but output pin to output pin route-throughs also exist). As Figure 2.6 shows, a site route-through is made up of configured site PIPs, LUT route-throughs, and FF route-throughs.

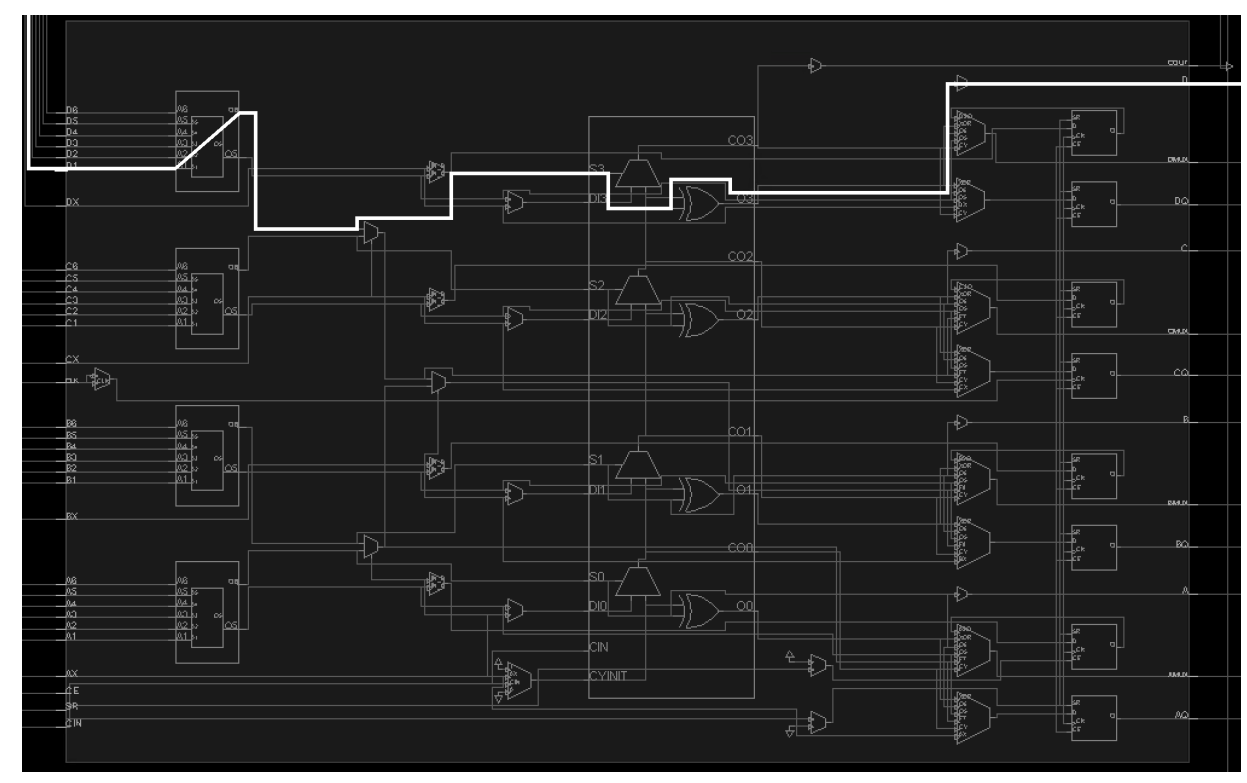

Figure 2.6: A SLICEL Being Used as a Site Route-Through

\subsubsection{Tiles}

The next level of hierarchy in a Xilinx 7-Series FPGA is the tile. A Xilinx device can be viewed as a 2-dimensional grid of tiles, which can be grouped into logic tiles and interconnect tiles. Logic tiles include tiles such as CLB, DSP, and BRAM tiles and contain sites and BELs which implement logic. A CLB tile is the most common type of tile and contains two slices. Logic tiles of 
the same type are generally grouped into columns and are located next to columns of interconnect tiles.



Figure 2.7: An Interconnect Tile

Interconnect tiles, as shown in Figure 2.7, contain switch-boxes and make up the general routing fabric of the device. The switch-boxes in interconnect tiles connect the switch box to other wires within the same switch-box, wires in nearby switch-boxes, and wires in neighboring logic tiles. These connections are made by turning on PIPs within the switch-box. Interconnect tiles also contain tie-off sites, which can be used to drive neighboring logic tiles with VCC and GND.

Xilinx uses the concept of a node to describe a group of directly connected wires that span one or more tiles. Each wire has a different name, although they are a part of the same node, as demonstrated in Figure 2.8. A node begins at either a site output pin or a PIP and ends in one or more site pins and/or PIPs. 


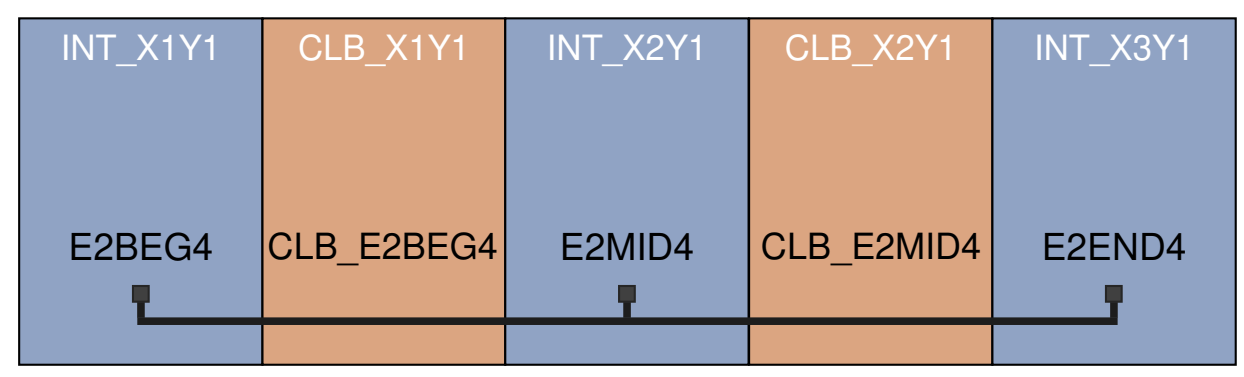

Figure 2.8: A Node - Reproduced from [4]

\subsubsection{Clock Region}

The highest level of hierarchy present in Xilinx 7-Series FPGA is the clock region. Xilinx divides its FPGAs into clock regions for clocking purposes. The number of clock regions present varies on device, but all Xilinx 7-Series FPGAs have clock regions that span 50 CLB tiles and one I/O bank, with a horizontal clock row in the center [15].

\subsection{FPGA CAD Flow}

Closely related to the development of FPGA architectures are FPGA CAD tools. Vendors of commercial FPGAs, such as Xilinx, spend considerable resources in the development of CAD tool suites so their customers can create high-quality designs. These CAD tool suites implement the FPGA CAD flow for their FPGAs, taking in a user-generated description of a circuit and creating a programmable bitstream for a specific target FPGA. The traditional FPGA CAD flow, shown in Figure 2.9a, consists of synthesis, technology mapping (tech-mapping) packing, placement, routing, and bitstream generation.

The first step, synthesis, takes in a user's register-transfer level (RTL) circuit design as input; this design is normally expressed in the form of a hardware description language (HDL), such as Verilog or VHDL. The synthesis process takes this RTL circuit and translates it into a gate-level netlist. A netlist is a listing of all of a circuit's components, often called cells, and their connections, called nets. In the next step of the CAD flow, tech-mapping takes the gate-level netlist and translates it into a netlist composed of LUTs, FFs, and other primitives available on the target FPGA. 


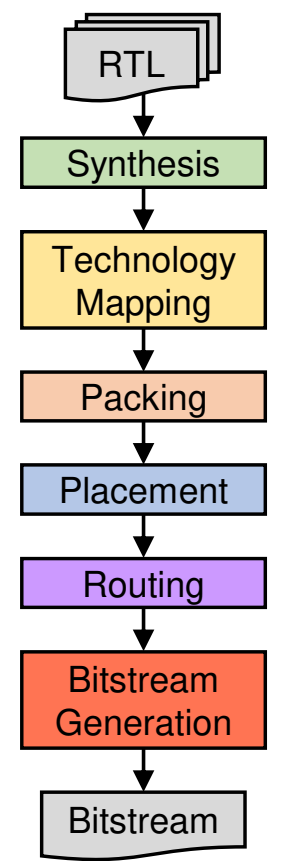

(a) Traditional CAD Flow

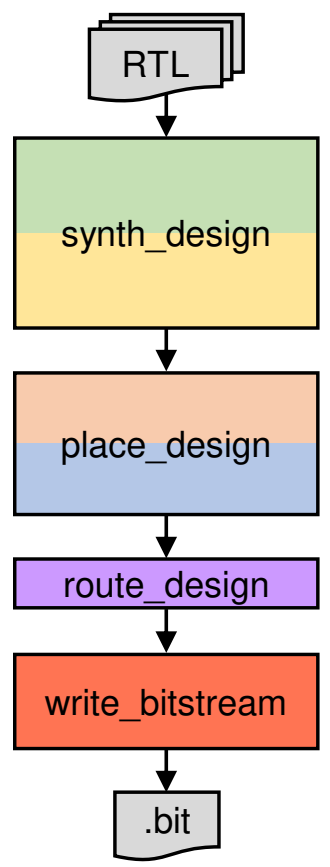

(b) Xilinx Vivado CAD Flow

Figure 2.9: Correspondence Between the Traditional and Vivado CAD Flows

The physical implementation steps, packing, placement, and routing, come next. These steps are all performed with certain goals in mind, such as minimizing the required wiring and maximizing circuit frequency. Packing, or clustering, can be viewed as a preparatory step for placement. In packing, closely related cells are grouped together into relatively-placed clusters. During placement, these clusters are physically assigned to unique logic blocks of the FPGA. Routing then makes all of the connections specified by the nets in the netlist, connecting the placed clusters to each other and to external I/O blocks.

Finally, bitstream generation converts the placed and routed circuit into a bitstream for the target FPGA. This bitstream can be programmed into the FPGA, causing the FPGA to be configured as specified by the user design. The actual format of the bitstream and the methods of programming it to an FPGA depend upon the specific device and its vendor.

\subsubsection{Vivado CAD Flow}

Xilinx's CAD Suite, the Vivado Design Suite, follows the traditional CAD flow for the most part. As Figure 2.9b shows, Vivado takes in an RTL design, usually expressed in an HDL, 
as input, just as the traditional flow. However, Vivado combines the synthesis and tech-mapping steps into the synth_design command. This command creates an electronic design interchange format $(E D I F)$ netlist, containing nets and Xilinx family-specific cells. The nets and cells in an EDIF netlist contain properties such as LUT equations and other configurable options.

Vivado also combines the packing and placement steps into one command, place_design. Once this command finishes executing, all the cells from the netlist will be assigned to unique BELs. Additionally, this command performs all routing within sites; this is called intra-site routing. Finally, the route_design command finishes connecting all the nets by performing inter-site routing, creating routes that connect site output and input pins.

The final step of Vivado's CAD flow is bitstream generation, which can be performed with the write_bitstream command. This command produces a bitstream as a bit file that can be programmed to the target Xilinx FPGA.

\section{Xilinx Bitstream Format}

Xilinx bitstreams for full devices are organized as shown in Figure 2.10. The header, which contains information about the bitstream like the name of the FPGA and a timestamp, comes first [16].

The next two sections of the bitstream contain configuration frames. Frames contain a bitstream's configuration data and consist of groups of 32-bit words; Xilinx 7-Series devices contain 101 words per frame. A standard bitstream first contains a section for CLB, I/O, and CLK configuration frames (block type 0), followed by a section for Block RAM frames (block type 1) [17].

The final section of the bitstream is the footer. Normally, the footer causes an expected, device-wide cyclic redundancy check (CRC) code to be compared against a calculated one. However, this check can be disabled by executing the Vivado Tcl command [set_property BITSTREAM.GENERAL.CRC Disable] before writing the bitstream. This command actually replaces writes to the FPGA's CRC register with a command that resets the CRC register [18]. Either way, the footer lastly contains commands that cause the FPGA to begin its startup sequence [19]. 


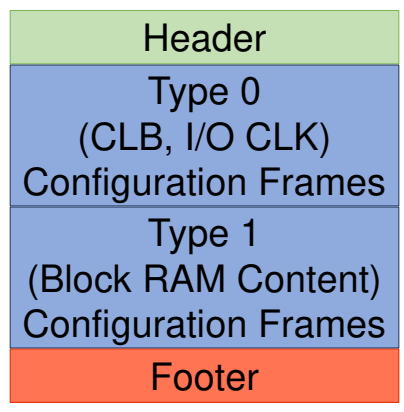

Figure 2.10: Organization of Standard Xilinx Bitstreams

\subsection{Partial Reconfiguration}

As previously mentioned, partial reconfiguration (PR) is a technique that allows for portions of an FPGA to be dynamically reconfigured at runtime while the rest of the device continues to operate. The use of PR has several benefits, including potential area savings and faster reconfiguration times. While there are many advantages to PR, not all FPGA vendors tools support it and those that do offer limited support.

Various approaches to PR have been explored by both academics and FPGA vendors over the years [20]. Several of these approaches have explored different levels of granularity for PR, from the LUT-level to large modules of logic. Module-based PR is the most common level of granularity for PR.

In module-based PR, large portions of design logic, known as reconfigurable modules $(R M s)$, are swapped in and out of reconfigurable areas of the FPGA, known as PR regions. These RMs are programmed to the FPGA with partial bitstreams, where a partial bitstream exists for each RM. The rest of the FPGA, known as the static region, hosts the unchanging part of the design, called the static design. An important consideration in module-based PR is how the static and reconfigurable parts of the system communicate.

Module-based PR has been further classified into three unique configuration styles: island style, slot style, and grid style [21]. These styles, visualized in Figure 2.11, are helpful in understanding the design concerns and limitations that come with PR.

In the island style, the PR regions are islands surrounded by the static region. Only one RM can occupy a PR region at a time in this style. A PR region must contain all of the resources that its largest RM requires; this often results in unused reconfigurable area, as shown in Figure 2.11a. 


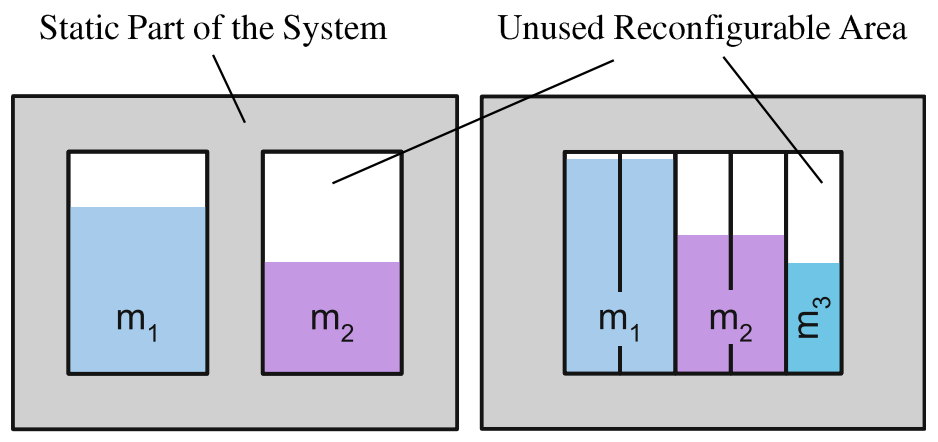

(a) Island Style

(b) Slot Style

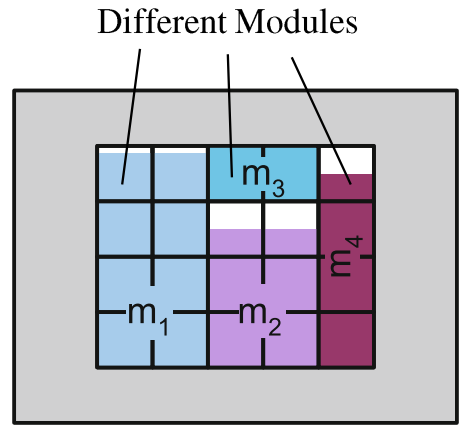

(c) Grid Style

Figure 2.11: Module-Based PR Configuration Styles

This style can be further sub-divided into the single island and multi-island styles. In single island style, a set of placed and routed RMs can only be used in one specific island. By contrast, placed and routed RMs can be used in different islands in multi-island style. The single island style is the only style currently supported by Xilinx and Intel [21].

The slot and grid styles come with more flexible module placement and more efficient usage of resources. Additionally, RMs can be relocated in both of these styles, as in the multi island style. In the slot style, as shown in Figure 2.11b, each PR region is divided into vertical slots of fixed size, allowing RMs to use multiple adjacent slots to meet their resource needs efficiently. The grid style, shown in Figure 2.11c, takes this idea a step further, dividing each PR region vertically and horizontally into a grid. While these styles have advantages, they come with additional implementation complexity and also require an increase in communication overhead [21].

\subsubsection{Xilinx Partial Reconfiguration Flow}

Since 1996, Xilinx FPGAs have been capable of some kind of PR, with support improving over the years [20]. Xilinx eventually began supporting module-based PR with PlanAhead, a tool in the Xilinx ISE tool suite [22]. Xilinx's current tool suite, Vivado, includes a PR flow that is very similar to the one introduced with PlanAhead.

As Vivado uses a module-based PR flow, a full Vivado PR design contains a static region and one or more PR regions, referred to as reconfigurable partitions in Vivado. Vivado's PR flow uses the single island configuration style, so placed and routed RMs can only be assigned to a specific PR region, as visualized in Figure 2.12. A configuration is a complete design that has 


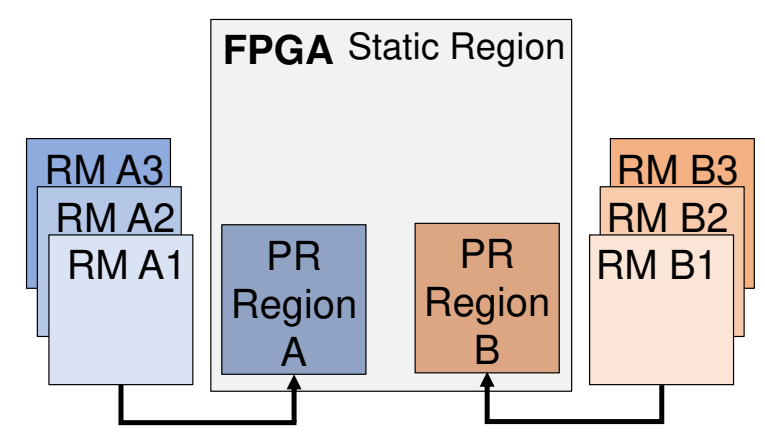

Figure 2.12: Vivado's Single-Island PR Style

one valid RM assigned to each PR region. PR designs can be implemented through both Tool Command Language (TCL) commands and the Vivado graphical user interface (GUI), though this text focuses on the Tcl commands. This section summarizes the steps of the Vivado PR flow, explained in more detail in [23].

\section{Synthesis}

The first step in Vivado's PR flow is to synthesize the HDL for the static design and the RMs separately. The static design acts as the top-level design and must contain a black box for every PR region; to do this, Verilog module or VHDL entity definitions are made for each PR region but contain no logic. The static design can be synthesized in Vivado with the regular synth_design command.

Since each RM can only be assigned to one PR region, each RM intended for a specific PR region must share the same module or entity name. Additionally, each of these RMs must have a completely identical I/O interface, even if some I/O signals are unused in some RMs. The RMs are synthesized in Vivado using the synth_design command with the -mode out_of_context switch. The out_of_context switch marks the design as being out of context for subsequent CAD steps and prevents I/O buffer insertion for the RM's interface signals.

\section{Floorplanning}

In the floorplanning step, physical constraints are created for each PR region, determining their size and location on the FPGA. This is done by creating a Pblock rectangle for each PR 
region. The Pblocks for the PR regions must follow certain rules, which are dependent upon the device architecture. The rules that must be followed for 7-Series FPGAs are as follows:

- The Pblock must contain only valid types of reconfigurable resources. Valid types of resources include all resources found within slices (LUTs, FFs, etc.), block RAMs (BRAMs), FIFOs, and digital signal processing (DSP) blocks. All other types of resources, including clocks and clock-modifying logic (BUFG, MMCM, PLL, etc.), I/O and I/O related components, serial transceivers, and individual architecture feature components (BSCAN, STARTUP, etc.), must remain in the static design.

- The left and right boundaries of the Pblock must be placed between two logic tile columns (e.g., CLB-CLB, CLB-DSP) and not between two interconnect tile columns (INT-INT). This ensures that the placer and router are able to make the most efficient use of the logic resources as possible.

- In order to use the global set/reset (GSR) functionality in the PR region, the Pblock height must be aligned to clock region boundaries and the RESET_AFTER_RECONFIG property on the Pblock must be set.

- The Pblocks cannot overlap.

- Pblocks for PR regions cannot be nested.

The Pblocks for the PR regions can be created either in the Vivado GUI or with the create_pblock, add_cells_to_pblock, and resize_pblock Tcl commands. The HD_RECONFIGURABLE property of each Pblock must also be set to true. With this property set, later steps of the Vivado PR flow can tell that the Pblock defines a PR region, affecting their treatment of the area.

\section{Implementation of the Static-Only Design}

In this step, the static part of the design is placed and routed. In order to do this, a full initial configuration is required to be used as a placeholder. This means that an RM must be assigned to each PR region in the design. A synthesized RM can be assigned to a PR region using either the read_checkpoint or update_design commands with the -cell switch specifying the PR region. 
The first command allows a synthesized Vivado design checkpoint (DCP) for an RM to be assigned to the PR region while the second command does the same thing with an EDIF netlist.

Once all the PR regions have been assigned RMs, the place_design and route_design commands are executed, completing the implementation of the initial configuration. Additional commands must then be used to isolate the static design. First, the update_design command with the -cell and -black_box switches is used to remove an RM from a PR region; this must be done for all RMs in the design. Next, the lock_design command with the -level routing switch is used to lock down the placement and routing of the static design, which must remain consistent across all configurations. Lastly, the write_checkpoint command can be used to export the static-only design as a DCP.

Partition Pins The placement and routing performed on the initial configuration adds partition pins to the PR design. Partition pins are the points at which the static and reconfigurable logic interface. Logically, they act as top-level ports for the reconfigurable module. Physically, they are implemented as wire segments within the PR region [23].

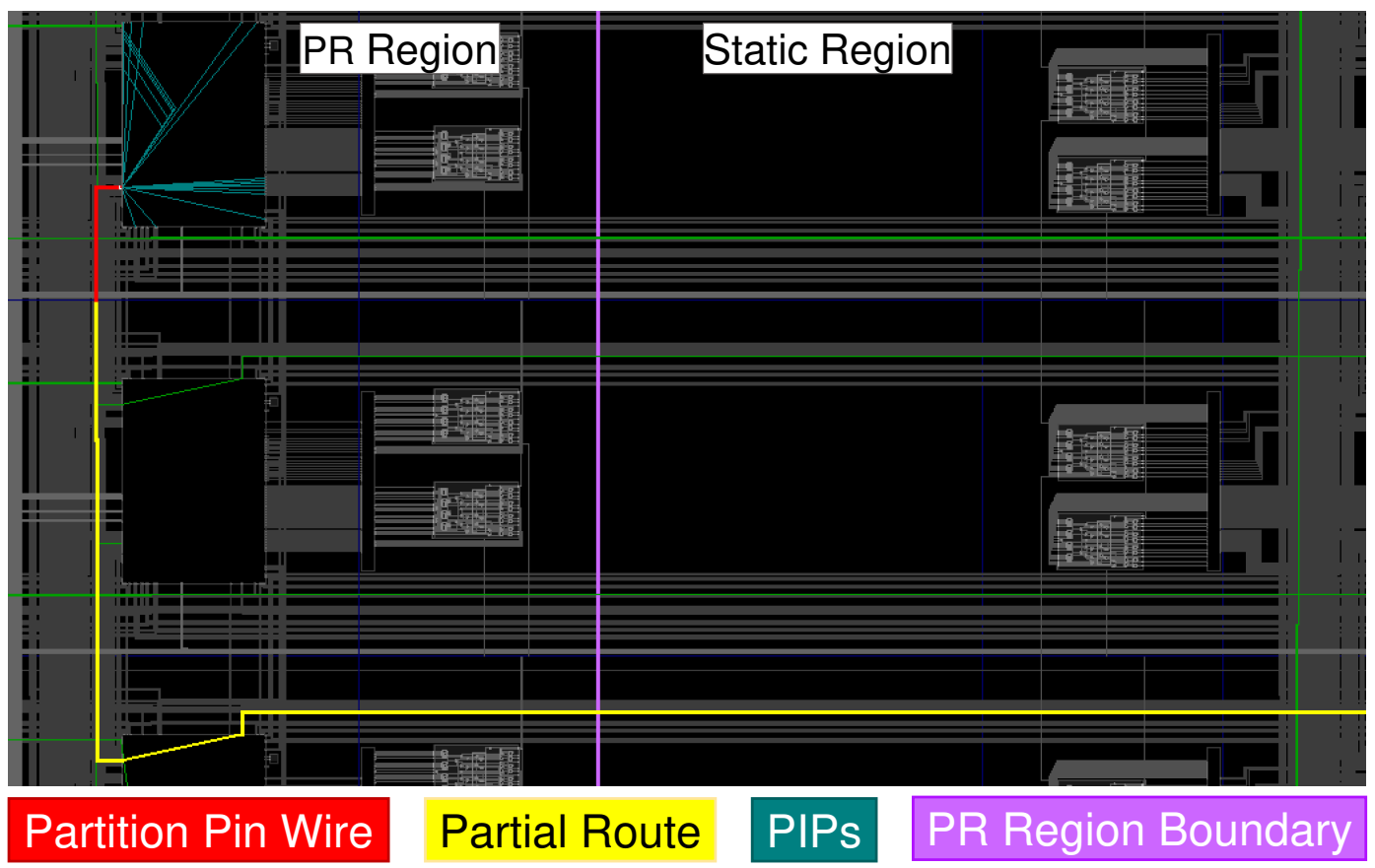

Figure 2.13: A Partial Route to a Partition Pin 
During placement and routing, Vivado automatically determines the placement of partition pins and creates routes connecting them and the static logic. Figure 2.13 shows an example of such a route in an isolated static design. The route begins at a partition pin and continues out to the static region. Because the RMs were removed to isolate the static design, the partition pin is un-driven; the PIPS in the interconnect switch-box show possible ways logic in the PR region could be routed to drive the partition pin. All partition pins and the partial routes connecting them to the static design must be identical for each RM. However, the completion of the partial route can be different in each RM.

Static Design Use of PR Region Resources While the static design and RMs are never allowed to use logical resources contained in the other's region, the static design may be required to cross PR regions in order to complete its routing. This most commonly entails using PIPs in the interconnect tile switch boxes. However, in some cases, the static design may need to use an entire site in a PR region as a routing resource, routing through it as discussed in Section 2.1.3. As with partition pins and their physical routes, any usage by the static design of a PR region's routing resources must be consistent for each RM. Importantly, each RM's partial bitstream must also include information about this static routing, even though it is not part of the RM design.

\section{Implementation of RMs}

Once the isolated static design has been obtained, additional RMs can be placed and routed. In order to do this, the desired synthesized RMs must be assigned to the PR regions in the static design, using either the read_checkpoint or update_design command. Note that an RM must be assigned to every PR region, making a full configuration. Once this has been done, the place_design and route_design commands can then be executed again to place and route the RM logic, while preserving the placement and routing of the static logic that was previously locked. The end result is a fully implemented configuration that can be saved to a DCP for later use. 


\section{Partial Bitstream Generation}

Full and partial bitstreams are generated using the fully implemented configurations. For each configuration, the write_bitstream command can be used to create a full bitstream as well as partial bitstreams for each RM in the design. A Xilinx FPGA must be initially configured using any one of the full configuration bitstreams, but any of the partial bitstreams can be programmed later during run time.

Partial Bitstream Format The partial bitstreams themselves are organized similarly to full bitstreams, the organization of which is seen in Figure 2.10. The main difference is that partial bitstreams' configuration frame section only contains frames that program the corresponding PR region. These frames include configuration data not only for the RM but also for any resources within the PR region the static design uses. Additionally, partial bitstreams may contain CFG_CLB (block type 2) frames and blanking frames.

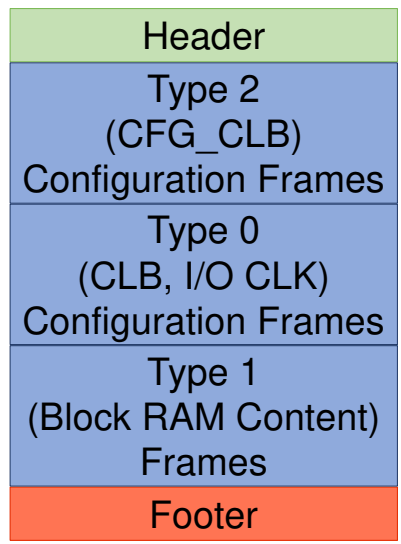

(a) Partial Bitstream

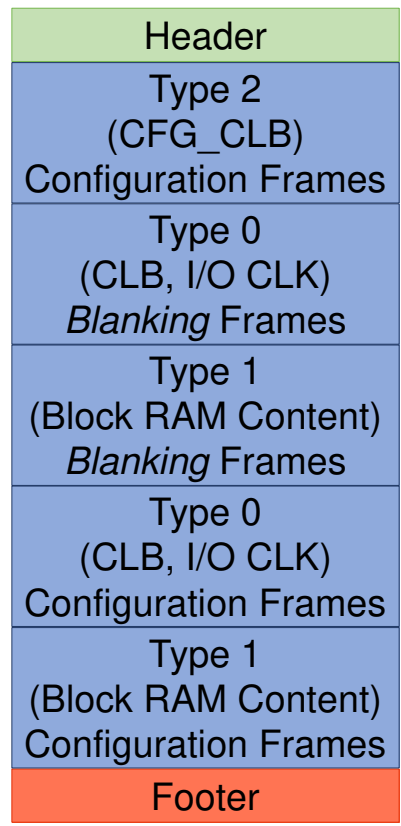

(b) Partial Bitstream with Blanking Frames

Figure 2.14: Organization of Partial Bitstreams 
Prior to Vivado 2016.1, partial bitstreams were organized as shown in Figure 2.14a. Like full bitstreams, partial bitstreams contain a header and a footer; these sections serve the same purposes as before. However, if the RESET_AFTER_RECONFIG property is set on the PR region, as is usually the case, CFG_CLB frames precede the other configuration frames [24]. CFG_CLB frames are mostly undocumented by Xilinx, but a comparison of different partial bitstreams reveals that the contents of this section of the bitstream differ for different PR regions [25]. Further comparison also reveals that this section is not dependent upon a PR design's I/O requirements; if two RMs with different I/O are made for two completely different PR designs, this section of the bitstream will still be the same. It can, therefore, be deduced that these frames define which portion of the FPGA is the PR region.

After Vivado 2016.1, blanking frames precede the normal configuration frames, as shown in Figure 2.14b. These frames were added to prevent potential glitch events that can occur when an RM is swapped into a PR region. Essentially, the configuration data in these frames replaces a placed and routed RM's configuration with tie-off LUTs [23]. Besides this, the partial bitstream format remains the same. 


\section{CHAPTER 3. THIRD-PARTY CAD TOOLS AND RELATED WORK}

Maverick relies on and extends a number of existing third-party CAD tools to create a full PR CAD flow. As such, this chapter gives an overview of these CAD tools and also reviews related work. Special attention is given to the CAD tools used by Maverick.

Several third-party CAD tools have been developed over the years, with some primarily being used for experimentation on hypothetical FPGAs, such as Verilog-to-Routing (VTR) [7], and others targeting commercial FPGAs. The tools that target commercial FPGAs fit within two main categories: (1) Cooperative CAD Tool Frameworks and (2) Stand-alone, Vendor-independent tools [26].

Cooperative CAD Tool Frameworks are frameworks which allow users to perform CAD tasks not readily possibly using the vendor tools. They typically are used to perform some portion of the FPGA CAD flow and rely on vendor tools to accomplish the rest of the flow. This means they must be able to interface with vendor tools, importing and exporting design and device data. Tools that fall into this category include RapidSmith [8] and Torc [9], which interface with Xilinx ISE, as well as RapidSmith2 [2] and RapidWright [10], which both interface with Vivado.

The second category of tools, the stand-alone, vendor-independent tools, can be used to "replace" the vendor tools. They aim to implement portions of the FPGA CAD flow and are sometimes combined to create full CAD flows for commercial FPGA devices. As such, they have no need to interface with the vendor tools and are typically not capable of doing so. Examples of tools in this category include Yosys [1], nextPnR [27], and Project X-Ray [3].

Various vendor-independent tools have been used and combined to create third-party flows similar to Maverick that can generate bitstreams for commercial FPGAs. For instance, the work in [28] described a proof-of-concept autonomous computing system running on the PowerPC of an embedded Virtex-II Pro device. This system could place, route, and generate partial bitstreams 
for technology mapped designs. For bitstream generation, it used an unreleased version of Xilinx's JBits.

Another toolchain, the IceStorm flow [29], is a full FPGA CAD flow for the commercially available iCE40 family of FPGAs from Lattice Semiconductor. It uses Yosys for synthesis, Arachne-pnr [30] for placement and routing, and the Project IceStorm tools for bitstream generation. This flow has enabled Trenz Electronic's icoBoard [31], a Raspberry Pi accessory containing an iCE40 FPGA. Using the icoBoard and a Raspberry Pi board, the IceStorm flow can compile designs to bitstreams on the Raspberry Pi's ARM CPU that can then be programmed onto the iCE40 FPGA. These bitstreams can then be programmed onto the iCE40 FPGA.

At the time of the writing of this thesis, SymbiFlow is intended to absorb the IceStorm flow and to become "a FOSS Verilog-to-Bitstream FPGA synthesis flow for Xilinx 7-Series, Lattice iCE40 and Lattice ECP5 FPGAs" [32]. SymbiFlow currently combines Yosys, VTR or nextPnr, and the Project X-Ray tools, Project IceStorm tools, or Project Trellis [33] tools to generate bitstreams for Xilinx 7-Series, Lattice iCE40, and Lattice ECP5 FPGAs, respectively.

Maverick shares many similarities with these flows, but uniquely uses and extends both cooperative CAD tool frameworks and stand-alone, vendor-independent tools to implement a PR CAD flow for Xilinx 7-Series devices. Specifically, Maverick uses Yosys for synthesis and techmapping, RapidSmith2 for the physical implementation steps, and Project X-Ray for bitstream generation. An overview of these tools will be given in the following subsections.

\subsection{Yosys}

Yosys Open SYnthesis Suite (Yosys) [1] is an open-source C++ framework for registertransfer level (RTL) Verilog synthesis. Similar to most software compilers, Yosys splits the synthesis process into three main phases: a front-end, a middle-end, and a back-end, as shown in Figure 3.1.

Yosys reads in a user's design data using one of its supported front-ends. At the time of this work, Yosys includes a Verilog front-end that has extensive Verilog-2005 support as well as support for a subset of SystemVerilog. The Verilog front-end consists of a preprocessor, a lexer, and a parser; together, these components scan and analyze the Verilog source code. The final 


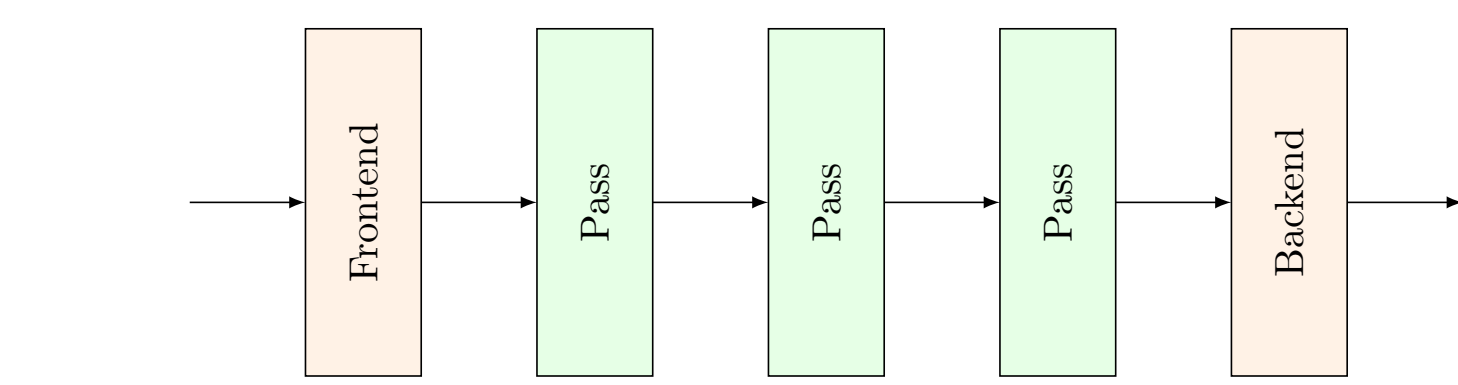

HDL

Internal Format(s)

Netlist

Figure 3.1: Yosys Phases. Taken from [5]

step of the front-end transforms the user design into Yosys' main internal data format, the RTL Intermediate Language (RTLIL).

The next phase of Yosys is the middle-end, which transforms the RTLIL representation of the design with a series of optimization passes. Architecture-specific tech-mapping passes can also be used to target specific architectures, such as the Xilinx 7-Series FPGAs. At the time of this work, Yosys cannot map to the complete set of Xilinx 7-Series primitive cells; for instance, digital signal processing (DSP) cells are completely unsupported. However, the set of supported Xilinx 7-Series cells is continually growing.

The last phase of Yosys is the back-end, which transforms the RTLIL representation into a suitable output format. One of the back-ends supported by Yosys is the EDIF format used by Vivado.

\subsection{RapidSmith2}

RapidSmith2 [2] is a cooperative CAD tool framework that builds upon the original RapidSmith [8]. RapidSmith2 is written in Java and offers an extensive set of useful APIs, data structures, and methods that allow users to manipulate Xilinx designs at the BEL and cell-level. RapidSmith and RapidSmith2 have been used in the creation of new CAD flows, in fault-tolerance applications [34], and in FPGA security research [35]. 


\subsection{RapidSmith2's Interface with Vivado}

Many CAD frameworks, including the original RapidSmith, relied on the Xilinx Design Language (XDL) interface included in Xilinx ISE. The ISE xdl executable allowed users to export Xilinx designs to the human-readable XDL design format at any point of the Xilinx design

flow. XDL designs could then be manipulated within external CAD tool frameworks, such as RapidSmith, and then re-imported back into Xilinx ISE.

The $\mathrm{xdl}$ executable also provided an option to generate complete human-readable FPGA device reports, known as Xilinx Design Language Routing and CLB (XDLRC) files. An XLDRC file contains a listing of all the physical components in a Xilinx FPGA, including BEL-level information and a detailed routing graph. XDLRC files are extremely verbose and are generally several gigabytes in size, with the largest devices taking over 100 GB of storage space. The original RapidSmith parsed XDLRC files to create more compact device models for its own internal use.

However, the XDL and XDLRC formats are not supported by Xilinx's current tool suite, the Vivado Design Suite, which instead includes a TCL interpreter. This makes external CAD tools that rely on these formats obsolete. RapidSmith2, therefore, uses Tincr [13] and the Vivado Design Interface (VDI) [36] to exchange device and design data with Vivado.

\subsubsection{Tincr}

Tincr [13] is a TCL plugin to Vivado that is made up of two main libraries: TincrCAD and TincrIO. TincrCAD is made up of commands that focus on implementing custom CAD tools directly in Vivado. TincrIO provides a set of commands for extracting device and design data from Vivado.

\subsubsection{Vivado Design Interface}

VDI [36] extended TincrIO with several additional commands and features to enable the exchange of device and design data with Vivado for Xilinx 7-Series, Ultrascale, and UltraScale+ devices. Figure 3.2 shows how with Tincr and VDI can be used to exchange device and design information between RapidSmith2 and Vivado. 


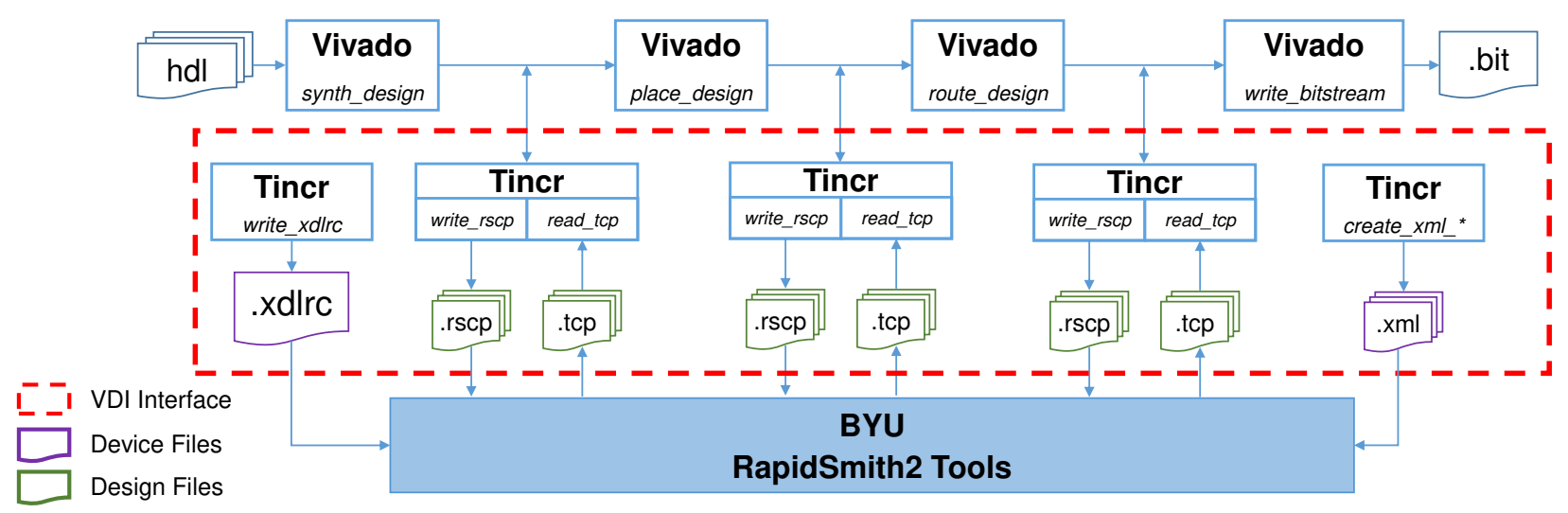

Figure 3.2: RapidSmith2 Tool Flow Using Tincr. Adapted from [4].

To extract device data from Vivado, Tincr's write_xdlrc command is used. This command mines information from Vivado and creates complete XDLRC files for any device supported by Vivado. Tincr also includes additional create_xml* commands, which extract additional information and create XML files needed to represent devices fully. As explained, XDLRC files are massive, making them impractical for regular use. Because of this, RapidSmith2 includes a Device Installer which reads in the XDLRC file and XML files to create a compact device file. Once a device has been installed within RapidSmith2, the XDLRC is no longer used, and the compact device file is instead used to provide a device model within RapidSmith2.

As shown in Figure 3.2, two checkpoint formats are used to exchange design information with Vivado. Two formats were created instead of a single one for a number of reasons, which are described in the RapidSmith2 technical report [4]. RapidSmith2 does not support hierarchical design, so designs must be fully flattened.

\section{RapidSmith Checkpoints}

Using Tincr's write_rscp command, synthesized, placed, and/or routed designs can be exported from Vivado into RapidSmith checkpoints (RSCPs). A standard RSCP consists of 6 individual files: design.info, netlist.edf, macros.xml, constraints.xdc, placement.rsc, and routing.rsc. Each of these files represents a specific aspect of a fully-flattened Vivado design for a full device. RSCPs can be imported into RapidSmith2, where the design can be modified. 


\section{Tincr Checkpoints}

Designs can be exported from RapidSmith2 as Tincr Checkpoints (TCPs), which can be imported back into Vivado using VDI's read_tcp command. A standard TCP consists of four files: netlist.edf, constraint.xdc, placement.xdc, and routing.xdc. As can be seen, TCPs are mainly made up of Vivado XDC files. These XDC files are made up of Vivado Tcl commands and can be imported quickly into Vivado through the use of Vivado's read_xdc. Just as with RSCPs, each of these files represents a specific aspect of a Vivado design for a full device.

\subsection{Project X-Ray}

Project X-Ray [3] is an open-source project that aims to document the Xilinx 7-Series bitstream format. It consists mainly of several $\mathrm{C}++$ tools and Python scripts. Project X-Ray uses scripts called "fuzzers" to create a large number of designs with specific varying properties. Bitstreams are generated for each of these designs and the Project X-Ray tools then cross-correlate the bitstreams to discover what the different bits of the bitstreams do.

Through the use of Project X-Ray, bitstream databases for Xilinx 7-Series FPGAs can be created. These databases consist of plain text files that contain mappings from device features to bits in the Xilinx 7-Series bitstream. At the start of this work, Project X-Ray targeted only a specific region of an Artix7 FPGA. Additionally, Project X-Ray was originally unable to document several important Xilinx 7-Series device features, including BRAMs and IOBs. However, throughout this work, Project X-Ray has grown to be able to document larger device regions, additional 7-Series devices, and more device features.

Project X-Ray and SymbiFlow also introduced the FPGA Assembly (FASM) file format. FASM files are human-readable text files that contain a list of low-level instructions to enable different features within an FPGA. However, these instructions are still at a high enough level of abstraction so as to hide many details about the bitstream format documented in the bitstream database. A sample FASM file is shown in Listing 3.1. This sample contains instructions to enable PIPs in the routing interconnect, set site PIPs, and configure a LUT equation. 
FASM files are mainly intended to be an export format from placement and routing tools. Project X-Ray contains additional tools that can be used in conjunction with a bitstream database to transform FASM files into bitstreams.

\section{Listing 3.1: Sample FASM File}

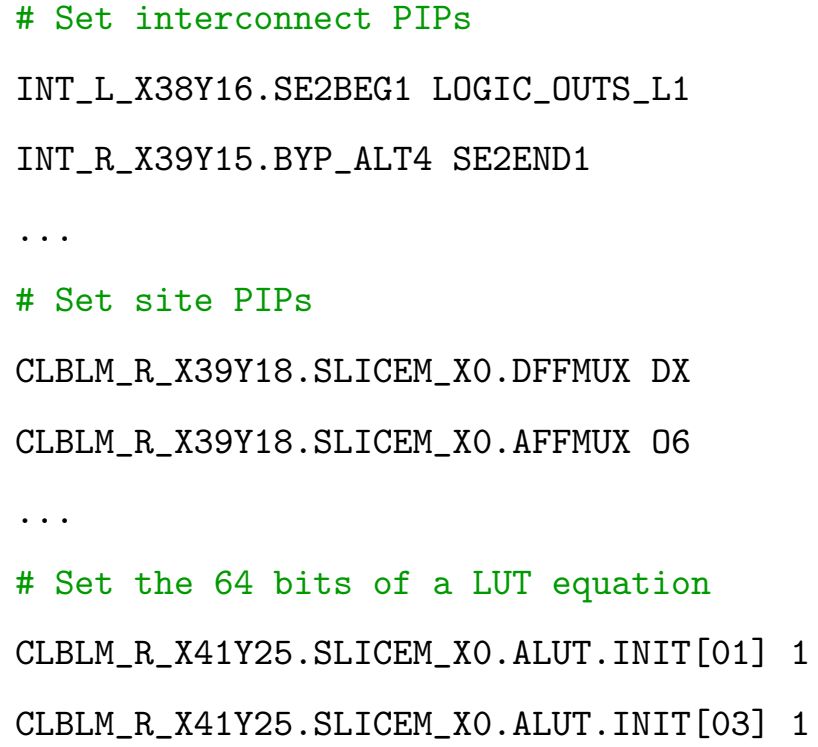




\section{CHAPTER 4. RAPIDSMITH2: PARTIAL RECONFIGURATION SUPPORT}

In order to support Maverick, several additions were made to RapidSmith2 and the Vivado Design Interface (VDI) to support partial reconfiguration (PR). This chapter describes the additions that were made as a part of this thesis. First, the additions made to support PR in RapidSmith2 are described. Then, the changes that were made to VDI to enable the import and export of RMs between Vivado and RapidSmith2 are described in detail.

\subsection{PR in RapidSmith2}

\subsubsection{Partial Device Representation}

As explained in Subsection 3.3.2, RapidSmith2 uses XDLRC files generated by Tincr. Using an XDLRC file as input, RapidSmith2 users can use the included device installer to generate compact device models for use within RapidSmith2. RapidSmith2 also now includes partial device installer written by Thomas Townsend.

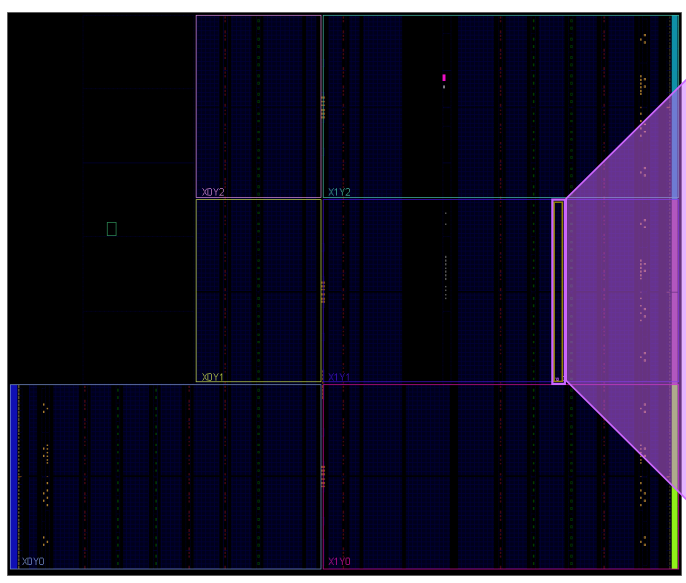

\begin{tabular}{|c|c|c|c|c|}
\hline CLB & INT_L & INT_R & CLB & $\begin{array}{c}\text { OOC } \\
\text { WIRE }\end{array}$ \\
\hline CLB & INT_L & INT_R & CLB & NULL \\
\hline CLB & INT_L & INT_R & CLB & NULL \\
\hline CLB & INT_L & INT_R & CLB & NULL \\
\hline CLB & INT_L & INT_R & CLB & NULL \\
\hline
\end{tabular}

Figure 4.1: Partial Device Representation in Tiles 
The partial device installer can be used to create smaller device models that represent a rectangular sub-region of a full Xilinx FPGA part. Unlike the full device installer, the partial device installer does not take an XDLRC file as input. Instead, it takes an already installed RapidSmith2 device file as input. Additionally, the partial device installer requires a name for the partial device, as well as the names of any two opposite corner tiles of the full device. As an example, Figure 4.1 shows a representation of a partial device in relation to the full xc7z020clg400-1 device. Here, CLBLM_L_X62Y50 and CLBLM_R_X63Y99 are the opposite corner tiles used to specify the partial device boundaries.

The partial device installer works by first copying several device-wide properties from the full device to the partial one. Next, the tiles, sites, and wires within the partial device boundaries are copied to the partial device. As can be seen in Figure 4.1, an extra column of tiles is also added when creating the partial device. The purpose of this column is to represent out-of-context (OOC) wires that are outside of the partial device boundaries but can programmatically connect to wires within the partial device. This allows users to still be able to work with programmable connections that enter or leave the partial device even though the partial device does not contain the tiles where those connections may start or end. All OOC wires are added to the upper rightmost "OOC_WIRE" tile and all other tiles in the rightmost column are set to null, so they take up as little memory as possible.

There are numerous benefits that come with using partial device models. Firstly, there is an obvious reduction in file size that comes with representing only a portion of an FPGA. Additionally, the memory requirements for all of the data structures needed by RapidSmith 2 to represent a device can be greatly reduced with the use of a partial device. This is particularly advantageous when RapidSmith2 is run on resource-constrained systems with limited memory.

Importantly, RapidSmith2 uses partial device models when working with RM designs to represent PR regions. This allows tools built within RapidSmith2 for full FPGAs to target PR regions without requiring significant changes. The RapidSmith2-based tools of Maverick take advantage of this, as described later in Chapter 6. 


\subsubsection{Partition Pins in RapidSmith2}

As explained in [4], RapidSmith2 includes an abstract CellPin class that represents the cell pins found in Vivado. Most CellPins in RapidSmith2 are instances of the BackedCellPin class, which represents cell pins on actual Xilinx primitive cells. To represent partition pins in RapidSmith2, the PartitionPin class, which extends the CellPin class, has been added to RapidSmith2.

PartitionPins differ from BackedCellPins in that they have an associated Wire object instead of an actual pin on a cell. Like BackedCellPins, PartitionPins also have an associated CellPinType and PinDirection. However, the CellPinType of a PartitionPin can only be PARTITION or PARTITION_CLK. The PARTITION_CLK type is used to distinguish PartitionPins that are driven by a global clock net; all other PartitionPins are of the PARTITION type.

PartitionPins are normally only created during the design import process. The creation of the PartitionPins during this process is further explained in Subsection 4.2.3.

\subsection{Reconfigurable Module (RM) RapidSmith Checkpoints (RSCPs)}

As explained in Subsection 3.3.2, VDI introduced two design representation formats. The first format is the RapidSmith Checkpoint (RSCP) format, which can be used to export designs from Vivado and import them into RapidSmith2 for manipulation. However, originally, only RSCPs for full-device designs were able to be created and used.

This section describes how the RSCP format has been extended to support reconfigurable module (RM) designs that have been synthesized, placed, and/or routed. First, an overview of the RM RSCP format is given. Next, the process of exporting RM RSCPs from Vivado is detailed. Then, the process of importing RM RSCPs into RapidSmith2 is explained.

\subsubsection{RM RSCP Format}

In order to support RMs, additional tokens were added to the RSCP format's routing.rsc file. Additionally, a new static.rsc file has also been added to the RSCP format. 


\section{Routing.rsc}

Following the routing of static design, details on the physical implementation of partition pins are available The following three tokens were added to the routing.rsc file to capture this information.

- PART PIN: Gives the name of the partition pin, the associated node, and the direction of the partition pin from the perspective of the static design. Listing 4.1 contains examples on lines 1 and 2. On line 2, the partition pin is named "text_i[16]", its associated node is "INT_R_X39Y38/SS2BEG3", and the partition pin acts as an output of the static design (and as an input of the RM).

- VCC_PART_PINS: Gives the names of all partition pins driven by VCC that are inputs of the RM. These partition pins do not have associated wires. Instead of the static design driving a wire, a VCC tie-off within the RM's PR region is used to drive the sinks of the partition pin. On line 4 of Listing 4.1, only "text $\mathrm{i}[13]$ " is a VCC partition pin.

- GND_PART_PINS: Gives the names of all partition pins driven by GND that are inputs of the RM. Like VCC_PART_PINS, these partition pins also do not have associated wires and are instead driven by GND tie-offs within the PR region. Line 5 of Listing 4.1 shows an example where "text_i[15]", "text_i[20]", and "text_i[23]" are all GND partition pins.

Listing 4.1: Sample routing.rsc

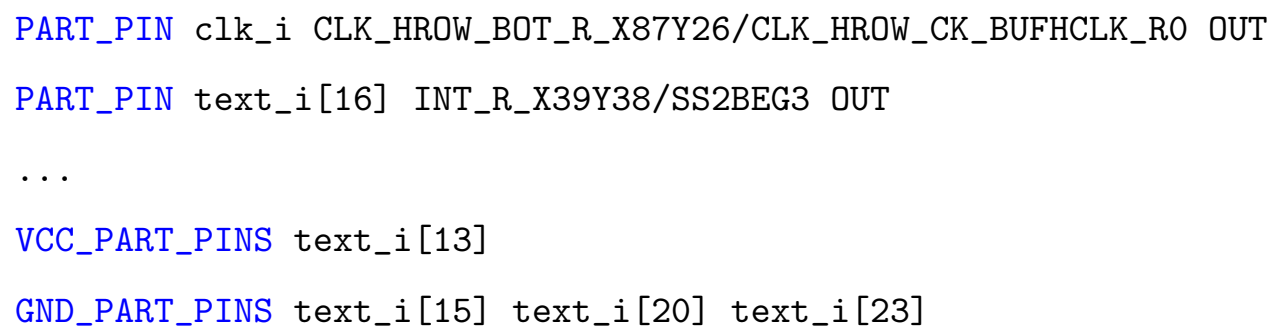

\section{Static.rsc}

The new static.rsc file was created to contain information about the static design that is needed when manipulating an RM. The static.rsc file contains the following tokens. 
- STATIC_RT: Contains the name of a static design net connected to one or more partition pins, the names of the RM net(s) connected to the same partition pin(s), and the partial route, determined during the implementation of the static design, in the form of a Vivado ROUTE string. In most cases, as in line 1 of Listing 4.2, a static design net connects to a single partition pin (and only one RM net connects to this same partition pin as well). However, it is possible for a static design net to drive multiple partition pins. Line 2 shows that the static design net "text $\mathrm{i}[16]$ " drives two RM nets, "text $\mathrm{i}[0]$ " and "text $\mathrm{i}[16]$ " (these nets share the names of the partition pins they connect to). The partial ROUTE string contains the routing of the net by listing each wire from the driver of the static net, "CLBLM_R_X37Y38/CLBLM_L_A", all the way to the individual partition pins of "text_i[0]" and "text_i[16]".

- SITE_RT: Gives the name of a site in the PR region that the static design has routed through as well as the specific route-through PIP. Line 4 of Listing 4.2 shows that the static design uses the "CLBLL_L_X40Y26/CLBLL_L.CLBLL_L_D5->>CLBLL_L_D” PIP to route through "SLICE_X61Y26”. A SITE_PIPS token and a SITE_LUTS token always accompany a SITE_RT token.

- SITE_PIPS: Names the same site that has been routed through and any site PIPs that must be enabled for the site route-through. Line 5 of Listing 4.2 shows that the 0 input of the DUSED routing BEL must be enabled.

- SITE_LUTS: Also gives the name of the site as well as any LUTs or flip-flops that must be routed through for the site route-through. Line 6 shows that the site route-through requires the D6 LUT to be routed from its A5 input to its O6 output.

- RESERVED_PIPS: Gives the name of any interconnect PIPs in the RM's associated PR region that the static design has used for its own routing, as explained in Section 2.3.1. Note that these PIPs to not include any PIPs used in the partial routes to and from partition pins. Line 8 of Listing 4.2 shows that the static design uses a PIP in the "CLBLL_L_X40Y26" tile that connects the "CLBLL_IMUX46" wire to the "CLBLL_L_D5" wire. 
Listing 4.2: Sample static.rsc

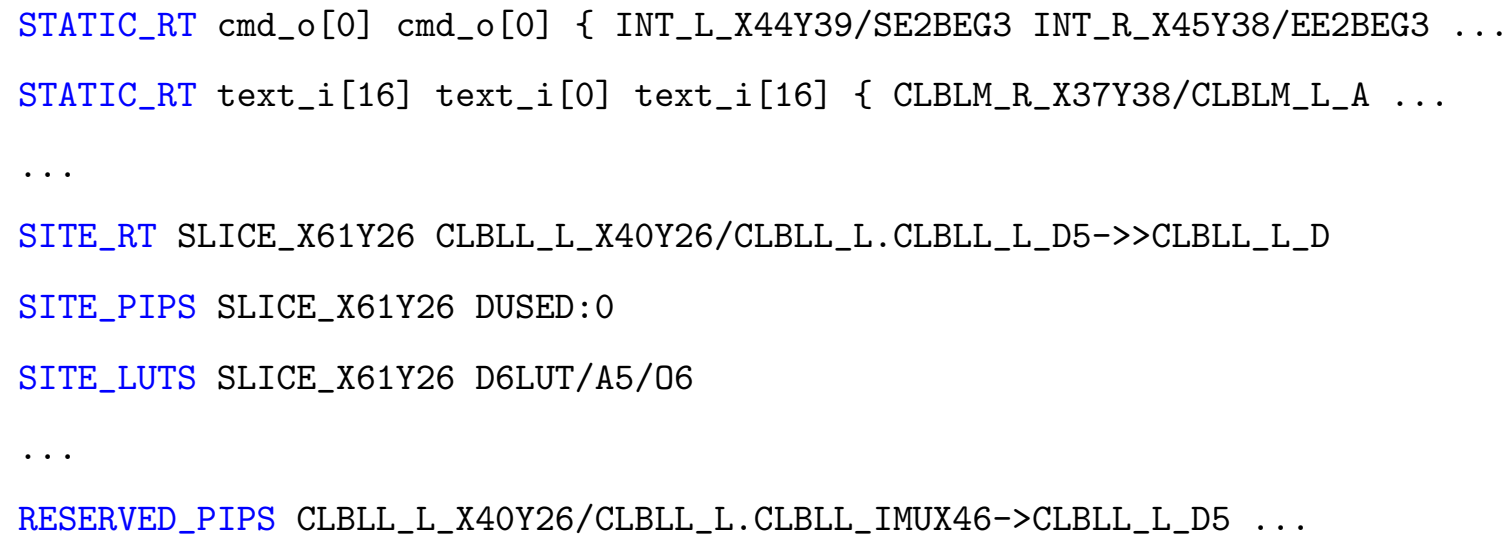

\subsubsection{Exporting RM RSCPs from Vivado}

The Tcl command tincr: : write_rm_rscp has been added to Tincr as an extension of VDI to enable the exportation of RM RSCPs. This command can be executed in Vivado on an out-of-context RM that has been synthesized, placed, or routed. Listing 4.3 shows the usage of this command.

\section{Listing 4.3: Tincr Command to Write an RM RSCP}

tincr::write_rm_rscp \$partialDeviceName \$staticDCP \$prRegion \$filename

The \$partialDeviceName argument specifies the name of the partial device that will represent the PR region in RapidSmith2. Next, the \$staticDCP argument specifies the path to the implemented static design DCP for the PR design, which gets generated as explained in Section 2.3.1. Then, \$prRegion specifies Vivado's name for the PR region that the RM belongs to, and lastly, \$filename specifies the filename for the output RM RSCP.

Much of RM RSCP generation is identical to standard RSCP generation. However, the generated design.info file of RM RSCPs includes that the design mode is "reconfig module" and also includes the name of the partial device. Additional work is also performed to generate the additional tokens in the routing.rsc file as well as the entire static.rsc file. The rest of this subsection describes how these files are created for RM RSCPs. 


\section{Routing.rsc}

General routing information in an RM RSCP routing.rsc file is generated in the same way as a regular RSCP. However, to create the PART_PIN, VCC_PART_PINS, and GND_PART_PINS tokens, the static design DCP is opened, and several Tcl commands are executed.

The PART_PIN tokens are first created. In order to get a list of all the partition pins with assigned wires in an RM design, the Vivado Tcl command [get_pins -filter HD. ASSIGNED_PPLOCS! =" "] is used. A PART_PIN token is created for each partition pin in this list. To obtain the name of the partition pin, the Vivado Tcl command [get_property REF_PIN_NAME [get_pins \$part_pin]] is used next. The HD.ASSIGNED_PPLOCS property on each partition pin gives the name of the assigned wire and is used to obtain the node with [get_nodes -of_object [get_wires \$wire_name]]. Then, the Vivado Tcl command [get_property DIRECTION \$part_pin] is used to get the direction of the partition pin.

Next, the VCC_PART_PINS and GND_PART_PINS tokens are obtained. Because the Vivado Tcl command [get_pins -filter HD.ASSIGNED_PPLOCS!=" "] only returns partition pins with assigned wires, the VCC and GND partition pins have to be obtained separately. To do this, the Vivado Tcl command in Listing 4.4 is used. This command gets all the pins of the PR region that are inputs and do not have assigned partition pins wires. Using either the global VCC or GND net in place of \$static_net gives the list of VCC or GND partition pins, respectively.

Listing 4.4: Vivado Tcl Command to Get VCC/GND Partition Pins



\section{Static.rsc}

To obtain the information described by the STATIC_RT, RESERVED_WIRES, and SITE_RTS tokens in the static.rsc file, several additional Tcl commands are executed with the static design open in Vivado.

First, the STATIC_RT token is created. The Vivado Tcl command [get_nets -of_objects [get_cells -hierarchical -filter IS_BLACKBOX == "TRUE"]] is used to obtain only the 
static nets that are connected to partition pins. To get the names of the RM nets that are connected to the same partition pins, the Vivado Tcl command [get_pins -filter \{HD.ASSIGNED_PPLOCS $!=" "\}$-of_objects [get_nets $\$$ net]] is then used on each net. Lastly, report_route_status -of_objects \$net is used on each net. As the example in Listing 4.5 shows, this command outputs a detailed listing of a net's route, including each wire and PIP used. The Tcl regular expression “ $(\{?) \backslash * \backslash \mathrm{s} *(\} ?)] ? \mathrm{p} ? \backslash \mathrm{s} *\left(\left[\mathrm{~A}-\mathrm{ZO}-9_{-} /\right]+\right) \backslash(\backslash \mathrm{s} *[0-9]+\backslash)$ " is used on each line of the output to obtain the node name on each line, along with any curly braces if they are present. The wire names and curly braces from each line are combined to create a Vivado ROUTE string, which is used by VDI to exchange routes between Vivado and RapidSmith2 [4].

Listing 4.5: Abbreviated Example Output of the get_route_status Command

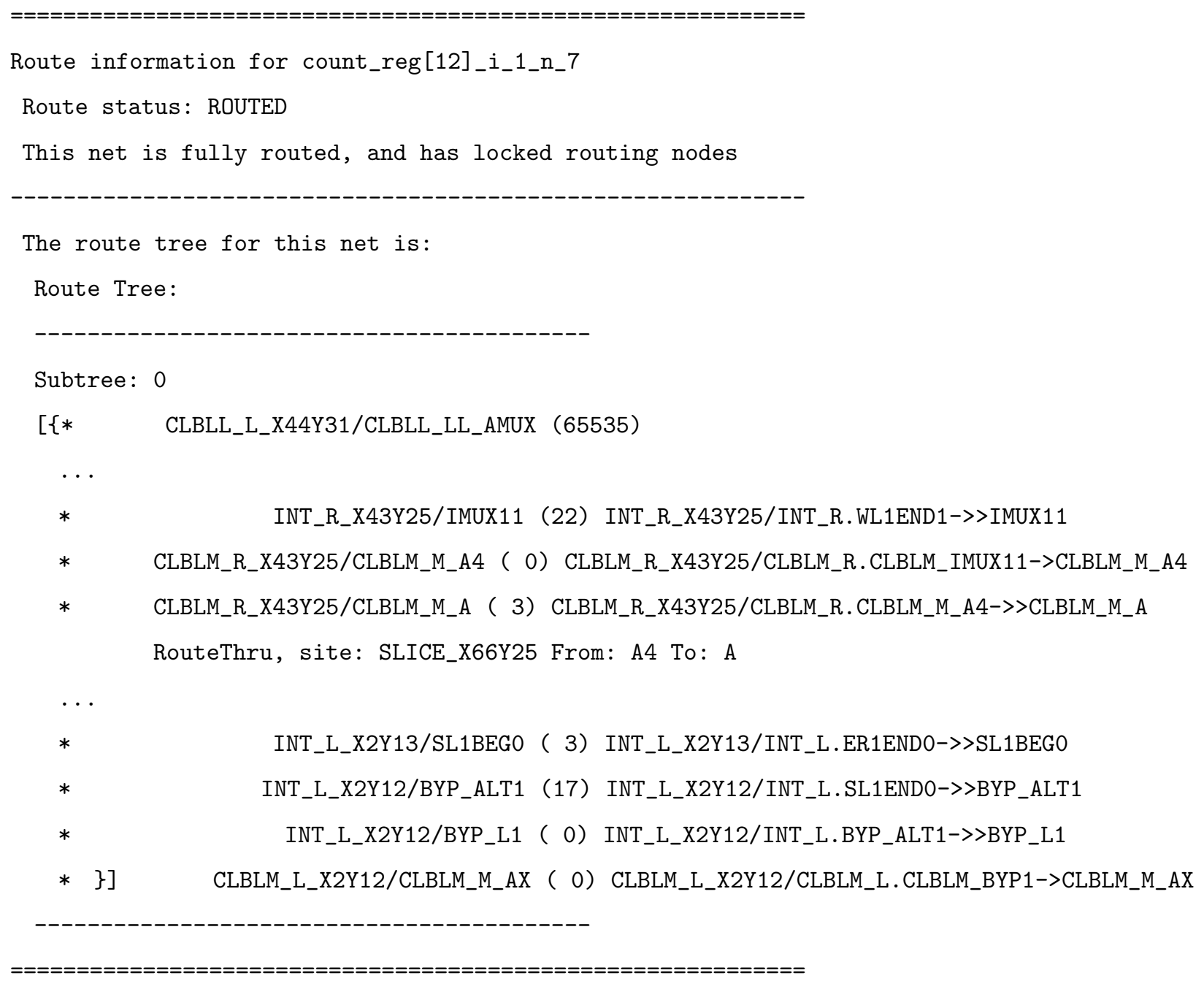


Next, the SITE_RT, SITE_PIPS, and SITE_LUTS tokens are generated. The IS_PSEUDO property of PIPs can be used to distinguish between regular PIPs and site route-throughs [14]. Therefore, this property is used to identify all sites within the PR region that the static design has routed through, as well as their associated PIP. Once this has been obtained, the used site PIPs are easily obtained with Vivado's get_site_pips command. The LUT and flip-flop route-throughs within the site are then obtained using the same general process outlined in [4].

Finally, the RESERVED_PIPS token is generated. Several standard Vivado Tcl commands are first used to obtain a list of static design PIPs that pass through the PR region and that are also not used in any routes connected to partition pins. Each uni-directional PIP that meets this criterion is included in the RESERVED_PIPS token. Each bi-directional PIP that meets this criterion requires further processing, as the direction that is actually used in the design must be determined. Vivado's bi-directional PIP objects contain no helpful properties for this purpose. However, Vivado's get_pips Tcl command can be used with a downhill flag to get the downhill PIPs from a specified PIP. This command is therefore used to identify the actual used direction of each bidirectional PIP. Once this direction is identified, each bi-directional PIP, with its used direction, is included in the RESERVED_PIPS token.

\subsubsection{Importing RM RSCPs into RapidSmith2}

RapidSmith2 has also been extended to import RM RSCPs. RSCPs are imported into RapidSmith2 with either the Vivado Interface or the similar Yosys Interface (described in Section 6.1). As with exporting RCPs, many of the steps to import an RM RSCP are the same as importing a standard RSCP. RapidSmith2 determines if an RM is an RM RSCP by reading the design.info file. If this file specifies that the RSCP mode is "reconfig_module", the partial device specified in the same file is instead loaded of the full device. Additionally, for RM RSCPs, RapidSmith2 must perform additional actions in the parsing of the routing.rsc file and must also parse the static.rsc file. The rest of this subsection explains RapidSmith2's parsing of these two files. 


\section{Routing.rsc}

For the most part, the routing.rsc in an RM RSCP is processed the same as in a standard RSCP. However, additional work is performed to parse the RM routing.rsc's PART_PIN, VCC_PART_PINS, and GND_PART_PINS tokens.

First, the PART_PIN tokens are parsed. For every PART_PIN, a PartitionPin is created and is connected to its corresponding net. Additionally, all of the wires in the partition pin's node are added to a mapping from nets to wires that have been reserved for exclusive use by the net(s). For many partition pins, no additional work is necessary. However, as described in [23], different partition pin usage situations can arise which require additional measures to be taken. These measures ensure that all RMs for a given PR region are consistent with one another. RapidSmith2 models its behavior for these situations after Vivado. There are five possible cases for partition pin usage that must be handled:

1. Both the static and RM sides of the partition pin have active logic. This is the optimal situation in which no additional measures are taken.

2. The static side has an active driver, but the RM does not have any active loads. This situation is fairly common because not all RMs have the same I/O requirements. In this situation, a LUT1 buffer cell is inserted and is driven by the partition pin.

3. The static side has active loads, but the RM has a constant VCC or GND driver. This situation is also common since some RMs may not drive unused I/O. In this situation, a LUT1 buffer cell is inserted and is driven by a VCC/GND tie-off source within the RM. An additional net is then added with the LUT1 cell's output pin as the driver and the partition pin as a sink.

4. Partition pin A is driven by partition pin B. A LUT1 buffer is inserted and partition pin B's net is modified to drive it. A new net is then added, with the LUT1's output pin as a driver and partition pin A as a sink.

5. More than one partition pin is driven by the same RM net. This case is handled similarly to case 4. A LUT1 buffer is inserted for each partition pin, and the RM net is modified to 
drive each of these LUT1 buffers instead of the partition pins. Nets are then added for each LUT1 buffer, with the LUT1 output pins as the drivers and the partition pins as the sinks.

The VCC_PART_PINS and GND_PART_PINS tokens are parsed next. VCC and GND partition pins actually handle a sixth partition pin usage case, in which the static side of a partition pin has a constant VCC or GND driver. For each partition pin included in these tokens, a PartitionPin is created and added as a sink of the global VCC or GND net. As explained earlier, VCC or GND partition pins do not have associated wires and occur when the static side of a partition pin has a constant VCC or GND driver.

\section{Static.rsc}

RapidSmith2 must also parse and process the tokens in the static.rsc file of RM RSCPs. As explained earlier, this file contains STATIC_RT, RESERVED_WIRES, and SITE_RTS tokens.

First, each STATIC_RT token, which contains the name of a static net, its associated RM nets, and a partial ROUTE string, is processed. Mappings between the static and RM nets are easily created when this token is parsed. However, loading the partial route into RapidSmith2 requires more work.

Normally, the route of a net is represented with a RouteTree within RapidSmith2 [4]. A RouteTree is a tree data structure, visualized in Figure 4.2a, in which the nodes contain physical wires of the device as well as the physical connection that is used to reach them. However, the ROUTE strings in the STATIC_RT tokens contain the names of wires in the static region, which are not present in the partial device. Because of this, a standard RouteTree cannot be used to represent these routes.

Therefore, a new RouteStringTree class, visualized in Figure $4.2 \mathrm{~b}$, is used for these routes. A RouteStringTree is an easily manipulated tree structure just like a RouteTree, but each node instead contains only the name of a wire instead of a reference to the actual wire on the device. Accordingly, the ROUTE string in each STATIC_RT token is parsed to create corresponding RouteStringTrees. All wires within the tree are also added to the RM design's list of reserved wires with mappings to the RM net(s) that have reserved them. Note that RouteStringTrees are 


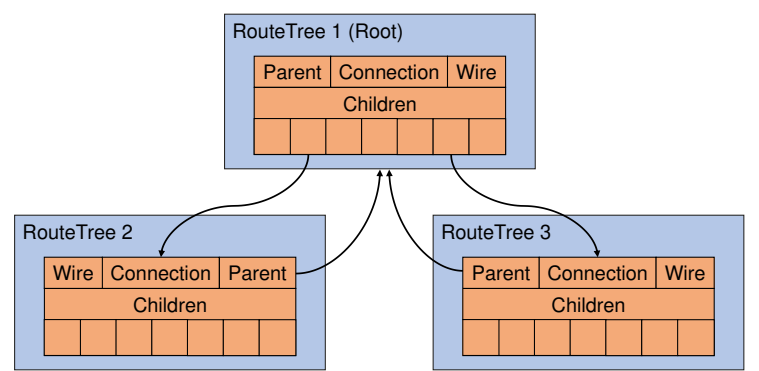

(a) RouteTrees - Reproduced from [4]

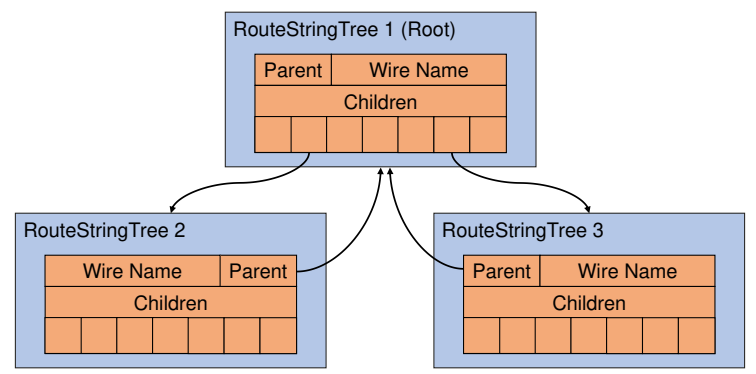

(b) RouteStringTrees

Figure 4.2: Visualization of RapidSmith2 Routing Data Structures

only created for routes expressed in STATIC_RT tokens; standard RouteTrees are still created for all other routes_-which are all completely contained within device boundaries.

After all the STATIC_RT tokens are parsed, the SITE_RT, SITE_PIPS, and SITE_LUTS tokens are parsed. RapidSmith2 saves each specified site to the RM's list of reserved sites, with the expectation that a RapidSmith2 user will check this list before trying to use the site for any other purpose. The Site PIPs specified in the SITE_PIPS tokens are also processed, setting RapidSmith2's internal site PIP data structures accordingly. Additionally, all LUT and flip-flop BELs included in the SITE_LUTS tokens are saved to a list for use by RapidSmith2 users.

The final token to be parsed is the RESERVED_PIPS token. Like the wires used in the partial routes of the STATIC_RT tokens, the wires that make up the PIPs are saved to the RM design's list of reserved wires. However, because these wires are used by nets out of the context of the RM design, these wires are not mapped to any nets within the design. Additionally, the reserved PIPs are also saved to a special list in the RapidSmith2 design so users can know exactly which PIPs were used by the static design.

\subsection{Reconfigurable Module (RM) Tincr Checkpoints (TCPs)}

The second design representation format introduced by VDI was the Tincr Checkpoint (TCP) format, reviewed in Section 3.3.2. TCPs can be exported from RapidSmith2 and imported into Vivado. Like RSCPs, only TCPs for full-device designs were originally able to be created and used. 
This section describes how the TCP format has been extended to support Maverick and RM designs that have been synthesized, placed, and/or routed. First, an overview of the RM TCP format is given. Next, the process of exporting RM TCPs from RapidSmith2 is detailed. Then, the process of importing RM TCPs into Vivado is explained.

\subsubsection{RM TCP Format}

In order to support RMs, additional types of Tcl commands are included in the routing.xdc file. Additionally, a new partpin_routing.xdc file has also been added to the TCP format.

\section{Routing.xdc}

Previously, VDI relied on Vivado to complete the intra-site routing of designs imported with TCPs; in most use-cases this works fine. However, issues can occur if a design is packed, placed, and routed within RapidSmith2; Maverick's RM phase does this with the designs it compiles. In this case, the results of the packing and placement will determine which site pins the router will route to and from. If such a design is then imported into Vivado without manually specifying the intra-site routing, the site pins that the router needs to connect may change. This can occur because the RapidSmith2-based packer may perform the intra-site routing of some sites differently than Vivado. In this case, the routed created in RapidSmith2 become invalid as they connect different site pins than what is expected by Vivado.

Because of this, two additional types of commands were added to the routing.xdc file to enable intra-site routing to be manually set. These commands come in pairs, with each specifying one of the following properties of a site:

- MANUAL_ROUTING: Used to specify the type of a site. This property must be set to manually route a site. Lines 1 and 3 Listing 4.6 both show examples of setting a site's MANUAL_ROUTING property to the SLICEL site type.

- SITE_PIPS: Used to turn on a set of site PIPs. For example, line 2 of Listing 4.6 sets PIPS within SLICE_X39Y59, configuring the AUSED, BUSED, and CUSED routing muxes to use their 0 inputs. 
Although these types of commands were added to RM TCPs to support the packing, placement, and routing of RMs, they can optionally be included in full-device RSCPs.

\section{Listing 4.6: Sample routing.xdc}

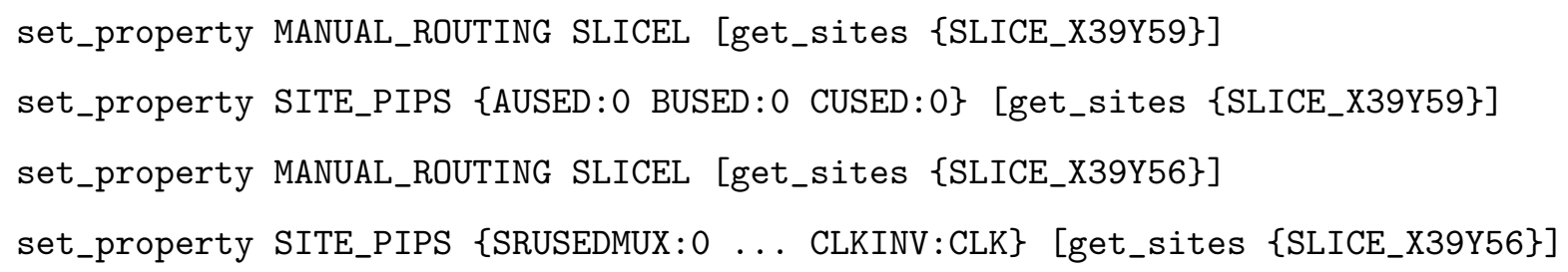

\section{Partpin_routing.xdc}

The new partpin_routing.xdc file was created to contain completed routes for nets that route to or from partition pins. Note that the routing.xdc file still contains completed routes for all other nets, whose routes are completely contained within the PR region. For every routed partition pin net, the partpin_routing.xdc file contains a Tcl command that sets the ROUTE string property of a net.

\subsubsection{Exporting RM TCPs from RapidSmith2}

TCPs are always exported from RapidSmith2 using its Vivado Interface. If the mode of the design in RapidSmith2 is set to "reconfig_module", an RM TCP is created instead of a standard TCP. This subsection explains how the additional intra-site routing commands are generated for the routing.xdc file and how the part_pin_routing file is created.

\section{Routing.xdc}

As long as the intra-site data structures of the RapidSmith2 design are accurately maintained, it is simple to generate both of the intra-site routing commands. The Vivado Interface simply queries for the type of each used site, writes a corresponding command, and then queries for and writes the used PIPs of each site. However, for two reasons, the Vivado Interface currently only writes intra-site routing commands for slices and no other site types. The first reason is that manually setting the intra-site routing on IOB sites causes Vivado to crash; it is possible this could 
occur for other site types as well. The second reason is that non-slice sites have very inflexible routing; it is likely not possible for a RapidSmith2-based packer to route a non-slice site differently than Vivado. If necessary, the Vivado Interface could be extended to write intra-site routing commands for additional site types in the future.

\section{Part_pin_routing.xdc}

Although RM designs treat partition pins as top-level ports, Vivado does not allow a net's ROUTE string to be manually set to a route that starts at a partition pin. However, the ROUTE string of nets involving partition pins can still be manually set in the context of a full PR design. Therefore, for each routed RM net, the Vivado Interface essentially merges the static portion of the net's route, which originates from the STATIC_RT tokens in the static.rsc RSCP file, with the RM's portion of the route.

Specifically, each routed RM net's RouteTree is transformed into a RouteStringTree. Then, if the RM net's route begins at the partition pin, all of the child nodes of the RM's RouteStringTree are added as children to the partition pin wire node of the static design's RouteStringTree. Conversely, if the RM net drives the partition pin, all of the children of the static design's RouteStringTree are added as children to the partition pin wire node of the RM's RouteStringTree. Either way, the merged tree is simply written as a Vivado ROUTE string to the part_pin_routing.xdc file.

\subsubsection{Importing RM TCPs into Vivado}

In order to import RM TCPs back into Vivado, the Tcl command tincr : :read_rm_tcp has been added to Tincr as an extension of VDI. This command can be executed to import a synthesized, placed, or routed RM TCP. Listing 4.7 shows the usage of this command. The \$staticDCP argument specifies the path to the implemented static design DCP for the PR design. Next, \$prRegion specifies Vivado's name for the PR region that the RM belongs to, and finally, \$filename specifies the file path and name for the RM TCP. 
tincr: :read_tcp \$staticDCP \$prRegion \$filename

Just as with the command for importing standard TCPs into Vivado, this command mainly works by using Vivado's read_xdc Tcl command. This makes the import process fairly simple. However, it should be noted that the read_xdc commands used to read the placement.xdc and routing.xdc files are executed with a -cells \$prRegion argument. This argument causes the Tcl commands contained in the xdc files to be executed within the context of the specified PR region. In contrast, the read_xdc command used to read the part_pin_routing.xdc file is executed without this argument since the Tcl commands in this file apply to the entire PR design. 


\section{CHAPTER 5. MAVERICK: STATIC DESIGN PHASE}

\subsection{Overview of Maverick}

As explained in Chapter 1, Maverick is a full flow for compiling reconfigurable modules (RMs) from Verilog to partial bitstreams. Maverick targets the Xilinx 7-Series family of FPGAs and is composed of third-party CAD tools, including Yosys, RapidSmith2, and Project X-Ray; these tools were all reviewed in Chapter 3. Additionally, Maverick relies on the Xilinx PR flow, explained in Chapter 2, and on the PR support added to RapidSmith2, detailed in Chapter 4. The components of Maverick can all be found online at https://github.com/byuccl/maverick.

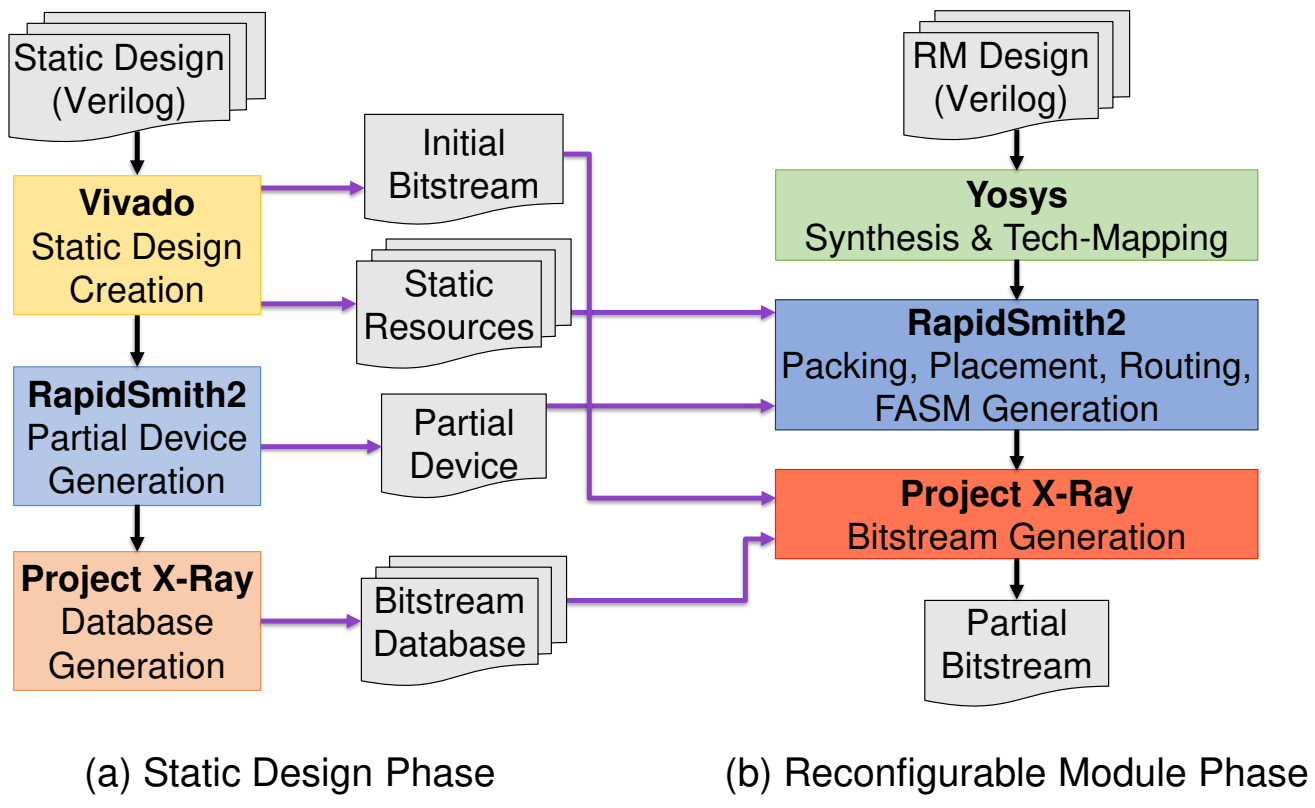

Figure 5.1: Overview of Maverick

As shown in Figure 5.1, Maverick is broken into two distinct phases: the static design phase and the RM phase. The static design phase, described in this chapter, relies on Vivado's PR flow to create an initial static design with a single target PR region. Tincr, RapidSmith2, and Project 
X-Ray are also used in this phase to produce a set of files that represent the static design and the target PR region. This phase must be executed on a computer capable of running Vivado.

In the RM phase of Maverick, Yosys, RapidSmith2, and Project X-Ray use the files output from the static design phase to compile an RM Verilog design to a bitstream. The RM phase of Maverick is unique in that it operates independently of Vivado and can run on resource-constrained embedded systems. This phase is explained in detail in Chapter 6.

\subsection{Static Design Creation: Vivado}

As shown in Figure 5.1a, the static design phase consists of three main steps: (1) static design creation, (2) partial device generation, and (3) bitstream database generation. These steps can be performed in any order, but are presented in the order shown in Figure 5.1 for ease of explanation.

The static design creation step relies on Vivado's PR flow, explained in Subsection 2.3.1. Accordingly, a PR design is created in Vivado, with the user creating a static design with a single PR region in the FPGA. This PR region must be created following the rules outlined in Subsection 2.3.1. An ideal PR region for Maverick should also only contain sites of type SLICEM or SLICEL, as other sites are not fully supported by the RM phase of Maverick.

Once the user obtains the placed and routed static design, multiple files must be generated for later use in the RM phase. First, the user must generate an initial full bitstream and an initial partial bitstream for the PR region with Vivado's write_bitstream command. The full bitstream is needed to initially configure the FPGA, while the partial bitstream will serve as the basis for the generation of subsequent partial bitstreams in the RM phase.

Next, the user must generate a routing.rsc and static.rsc file, collectively referred to as "static resources" in Figure 5.1. As detailed in Chapter 4, these files are created using new Tcl commands added to Tincr. These files include information about the partition pins, routes to the partition pins, and routing resources used by the static design. This information is vital for the RM phase of Maverick. 


\subsection{Partial Device Generation: RapidSmith2}

The next step is the generation of the partial device model for RapidSmith2, as explained in Subsection 4.1.1. This partial device must be generated such that it describes the exact same area of the FPGA as the PR region created in Vivado in the previous step.

The partial device model is used to represent the PR region in the RapidSmith2-based tools of Maverick's RM phase. Importantly, because the partial device model only describes the PR region, the memory required to represent the device in RapidSmith2 is also greatly reduced. This lower memory requirement is particularly advantageous when the RM phase is executed on resource-constrained systems.

\subsection{Bitstream Database Generation: Project X-Ray}

The final step of the static design phase is the generation of a Project X-Ray bitstream database. As explained in Section 3.4, Project X-Ray bitstream databases allow Xilinx 7-Series device features to be mapped to bits in the actual bitstream. Maverick's RM phase uses this generated database in conjunction with the earlier-generated initial partial bitstream to generate new partial bitstreams.

However, as noted in Section 3.4, Project X-Ray only supported the creation of bitstream databases for a specific region of an Artix 7 FPGA at the start of this work. Therefore, to accomplish one of the goals of this work, limited support for Zynq devices and partial bitstreams was added to Project X-Ray ${ }^{1}$. Specifically, the fuzzers in Project X-Ray were modified to target the region from tile CLBLM_R_X37Y0 to tile CLBLL_L_X44Y49 (8 cols x 50 rows of CLB and INT tiles) in the xc7z020clg400-1 Zynq device.

In order for partial bitstream generation to be possible with Project X-Ray, the CLB_CFG frames, described in Subsection 2.3.1, for the same Zynq device were also documented. Through observation of functional partial bitstreams, it was found that the CFG_CLB section of a partial bitstream for this device contains 74 configuration columns, with each column containing a single frame. This information was added to the bitstream database for the Zynq device; its use is further

\footnotetext{
${ }^{1}$ Independent from this work, support for larger regions and additional devices, including Zynq devices, has now been added to Project X-Ray.
} 
described in Section 6.7. Similar information would need to be added to other device databases to generate partial bitstreams for them.

Considering all of the above, Project X-Ray can be used to generate a database for any supported region of interest. Therefore, one option for a Maverick user is to generate a database describing only the targeted PR region. However, generating a database takes several hours even on relatively powerful computers. Waiting several hours to create a new bitstream database for every new PR region a user wants to target would be quite time-consuming. Instead, it is recommended to generate a bitstream database targeting the largest possible region of the target FPGA as possibleas long as this region contains the target PR region. While the generated database for a larger region will take up more disk space, databases documenting nearly entire devices are still quite lightweight. 


\section{CHAPTER 6. MAVERICK: RECONFIGURABLE MODULE PHASE}

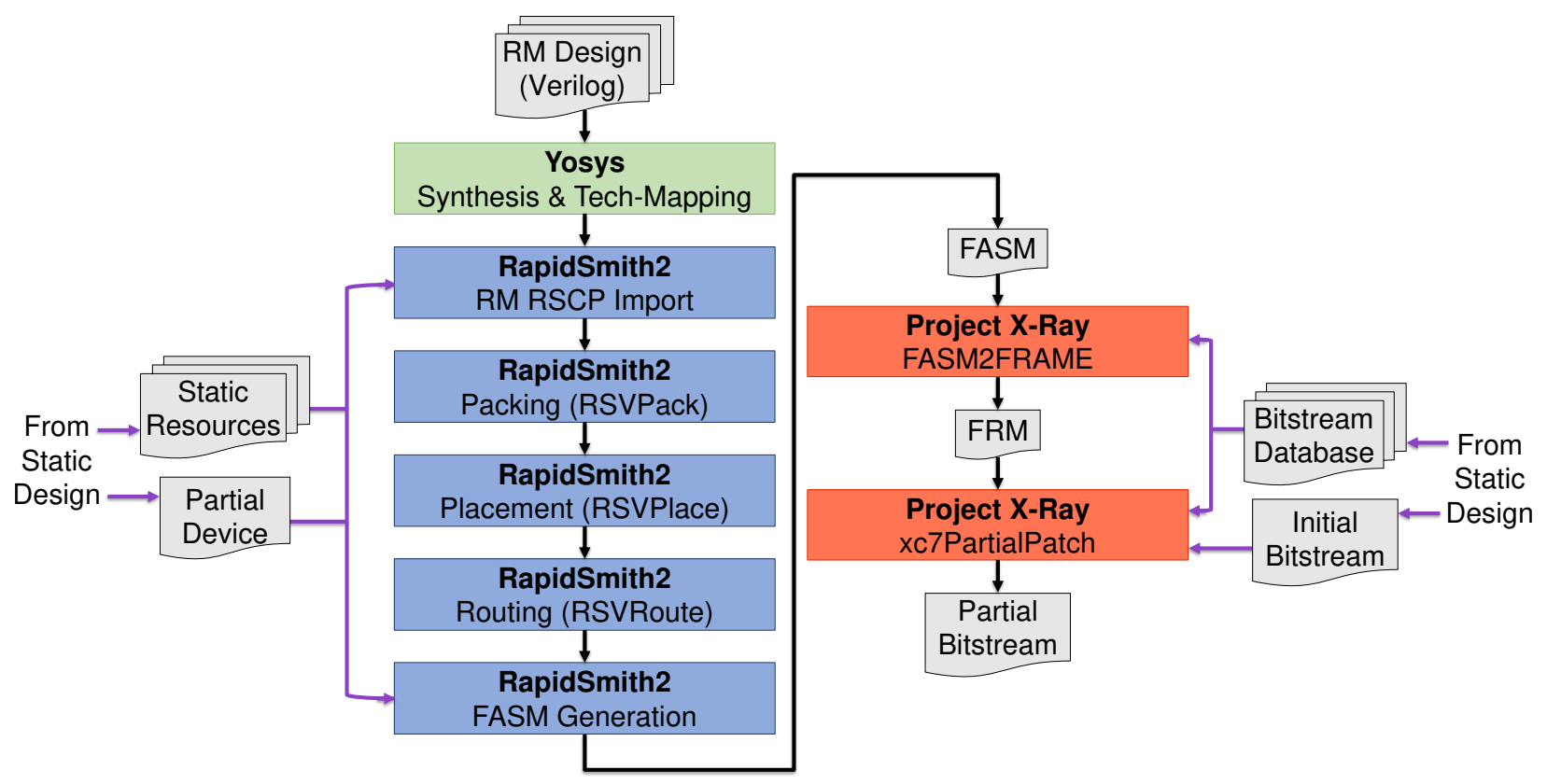

Figure 6.1: Detailed Overview of Maverick’s RM Phase

After the completion of the static design phase, all necessary files are available to support the RM phase of Maverick. The RM phase can then be executed and repeated to compile unique partial bitstreams for the target PR region.

Figure 5.1 gives a detailed overview of the individual steps of the RM phase. As can be seen, the RM phase of Maverick takes a Verilog design, targeting the PR region defined in the static design phase, as input. Yosys then synthesizes and tech-maps this design, producing an EDIF netlist as its result. This EDIF file, the static resources, and the partial device model are all then loaded into RapidSmith2. A collection of RapidSmith2-based programs then pack, place, and route the design. Once the design is routed, a final RapidSmith2-based program generates an FPGA Assembly (FASM) file. Project X-Ray tools then use this FASM file, along with the initial 
partial bitstream and bitstream database, to create a new and functional partial bitstream. The rest of this chapter describes these steps in more detail, detailing how they work together to create a complete tool flow for the target PR region.

\subsection{Synthesis and Tech-Mapping: Yosys}

The first step of the RM phase relies on Yosys to synthesize and tech-map the RM Verilog design. All RM Verilog designs should have a consistent I/O interface in order for Maverick to work as expected. Additionally, the writer of the Verilog design should be aware that Yosys does not support the full set of Xilinx 7-Series primitives. ${ }^{1}$ At the time of this work, Yosys included support for all LUTs, flip-flops, and carry-chain elements, as well as a small subset of block RAM and LUT RAM primitive cells. Yosys included no support for shift register LUT (SRL) cells, DSP cells, and other specialized Xilinx 7-Series primitives. The RM Verilog designs should be written with these expectations in mind.

Yosys includes a synth_xilinx command that combines several internal passes to synthesize, tech-map, and output an EDIF netlist for Xilinx 7-Series devices. Maverick uses the same passes as the synth $x$ ilinx command, but with two changes. The first change disables techmapping to BRAM cells of any kind due to a lack of support for BRAMs in Project X-Ray.

The second change was made due to the fact that Maverick uses Yosys to synthesize RM designs instead of full-device designs. As discussed, since all RM designs must share the same I/O, some I/O will not be used by some RMs. If some of these unused I/O are driven with a constant 0 and others are left un-driven in the Verilog, the EDIF netlist output by Yosys will create a unique GND net for each set. This goes against the expected style of Xilinx EDIF netlists, in which there is always a single global GND net. Because of this, Maverick executes Yosys with a setundef pass, with the -zero and -undriven options set. This setundef pass is run early in the synthesis process, so all undriven nets and undefined (x) constants are replaced with a constant 0 . As a result of this added pass, only one GND net appears in the EDIF netlist as desired.

\footnotetext{
${ }^{1}$ Yosys' supported set of Xilinx 7-Series primitives grew throughout the course of this work and is continually growing.
} 


\subsection{RapidSmith2 Design Import}

The next several steps of the RM phase rely on RapidSmith2 in order to physically implement the RM. Therefore, the EDIF netlist produced by Yosys, along with some files produced in the static design phase, must first be imported into RapidSmith2.

To import these files, the EDIF file is packaged into a new RM RSCP. The routing.rsc and static.rsc files produced in the static design phase are also added into this RSCP. Additionally, a design.info file, containing the name of the partial device, is added to the RSCP.

This RM RSCP is loaded into RapidSmith2 using a new Yosys interface. For the most part, the Yosys interface loads RSCPs the same way as RapidSmith2's Vivado interface. The partial device model referenced in the design.info file is automatically loaded and the information contained in the routing.rsc and static.rsc files is parsed as previously described. This includes saving the static design's partial routes to and from partition pins as well as marking resources used by the static design as reserved. However, the Yosys interface differs slightly from the Vivado interface in its parsing of EDIF netlists. This is due to the fact that although EDIF is a standard, different CAD tools produce their own "flavors" of EDIF. Yosys attempts to replicate Vivado's flavor of EDIF and is mostly successful; however, some differences remain. The Yosys interface was created to do any necessary handling of these differences.

Currently, the only difference in the handling of EDIFs done by the Yosys interface concerns carry chain cells. Specifically, the EDIF netlists produced by Yosys include Xilinx's legacy carry multiplexer (MUXCY) and carry XOR gate (XORCY) cells, while Vivado EDIFs use carry chain cells which internally include MUXCY and XORCY cells. Figure 6.2 shows the carry chain BEL present in Xilinx 7-Series FPGAs, the CARRY4 BEL, built of four MUXCY BELs and four XORCY BELs.

Whenever an EDIF netlist with MUXCY and XORCY cells is imported into Vivado, they are automatically transformed into carry chain cells, such as the CARRY4 cell. RapidSmith2's Yosys interface replicates this behavior for the Yosys-produced EDIFs. This is motivated in part by the desire to replicate Vivado's behavior in RapidSmith2 as closely as possible. But more importantly for Maverick, the RSVPack packer, discussed in the next section, cannot handle the legacy MUXCY and XORCY cells, necessitating their transformation into CARRY4 cells. 


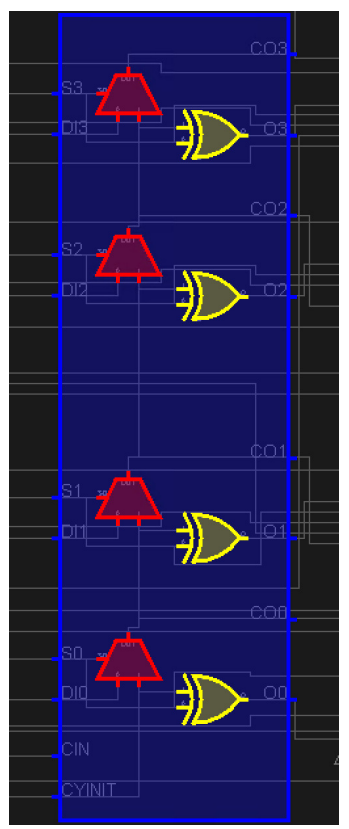

\section{CARRY4 XORCY MUXCY}

Figure 6.2: A CARRY4 BEL in Vivado with Internal MUXCY and XORCY BELs

\subsection{Packing: RSVPack}

Once the RM design is imported into RapidSmith2, it is packed using an existing RapidSmith2based packer called RSVPack [37]. This packer targets specifically Xilinx devices and works by repeatedly packing unplaced site clusters with cells.

More specifically, RSVPack begins each cluster by choosing a seed from the unpacked cells; RSVPack greedily chooses the cell with the most external pins. Once a cluster is created with an initial seed cell, RSVPack fills it with other cells, prioritizing cells that share nets with the cells already in the cluster. Each time a cell is added to a cluster, a series of tests is run to verify that the cluster is valid for the architecture. These tests include an efficient table-based routing feasibility checker that ensures the packed site cluster is internally routable without actually needing to route it. Once all the cells in the design have been packed, RSVPack performs the actual intra-site routing of all the site clusters. The results of RSVPack are then saved internally to data structures within RapidSmith2. These data structures are used by subsequent steps in Maverick, namely the placement of the RM design. 
In order for RSVPack to be used in Maverick, support for RM designs had to be added to RSVPack. This specifically involved slightly modifying RSVPack to handle the presence of partition pins in a design. Various components of RSVPack, including the previously mentioned table-based routability checker, consider the pins of different cells in their operation. As discussed in Subsection 4.1.2, partition pins are modeled as a special type of cell pin in RapidSmith2. However, unlike normal cell pins, partition pins do not actually belong to any cell. This necessitated the addition of various special partition pins cases to several RSVPack components. In addition to adding RM support, various small fixes were also made to RSVPack over the course of this work.

\subsection{Placement: RSVPlace}

Once packing completes, Maverick uses RSVPlace [37], also built on top of RapidSmith2, to place the site clusters onto specific sites inside the partial device. It first performs a quick random placement of the site clusters and then executes a simulated annealing placer using a wire-length based cost function. The cost function and annealing schedule used by this placer are both based on VPR's placer [38]. The final placement is saved to internal RapidSmith2 data structures. These data structures provide Maverick's router, RSVRoute, with the exact site pins that need to be routed together.

Little modification to RSVPlace was necessary for the purposes of Maverick. Due to the use of partial device models to represent PR regions, RSVPlace is automatically restricted to only be able to use sites within the PR region. However, RSVPlace was modified to not utilize any sites that were used by the static design and marked as reserved in RapidSmith2. RSVPlace could better support RM designs in the future by also considering the placement of partition pins as it places site clusters. As with RSVPack, a few bug fixes were also made to RSVPlace over the course of this work.

\subsection{Routing: RSVRoute}

Following placement, Maverick routes the design using a new router called RSVRoute, also built upon the RapidSmith2 framework, which can route both full-device and RM designs. Specifically, RSVRoute routes the design by turning on programmable interconnect points (PIPs) 
to route between pairs of site pins, and between partition pins and site pins. RSVRoute is primarily based on Pathfinder [39], an iterative, negotiation-based routing algorithm that has gained wide usage in both academia and industry since its introduction. Within RSVRoute's implementation of PathFinder, an $\mathrm{A}^{*}$ maze router [40] is used to quickly identify routes for nets.

Because RSVRoute was specifically created to enable Maverick, it will be discussed in much greater detail than RSVPack and RSVPlace. This section will first overview how RSVRoute prepares the nets before routing them. Next, the PathFinder algorithm, and RSVRoute's implementation of it, will be discussed. This will also include details on RSVRoute's A* maze router, which is used by PathFinder. Some of the current limitations of RSVRoute are then discussed. Finally, the unique features of RSVRoute that enable it to route RM designs will be briefly reviewed and highlighted.

\subsubsection{Preparing Nets for Routing}

Before the nets of the design are routed, RSVRoute first performs a number of preparatory steps. These steps result in the creation of IntersiteRoute objects, which contain helpful information for each inter-site net that needs to be routed. Most importantly, each created InterSiteRoute contains a start RouteTree and one or more sink RouteTrees.

\section{Start Trees}

Complete inter-site routes begin at either the output pin of a site or a partition pin; an intersite router can route an inter-site net by routing from this point. For each inter-site net that needs to be routed, RSVRoute creates a RouteTree that starts from this point. For many nets, a portion of the route starting at this point can be determined very easily; this portion of the net's RouteTree is henceforth refereed to as the net's StartTree. In such nets, only one PIP can possibly be used from the starting point of the route. PIPs such as this are continually added to the StartTree until the first branch in the routing interconnect is reached-in which there exists more than one possible connection for the route to take. Figure 6.3 shows an example of an inter-site route in Vivado where the StartTree begins at a site output pin and ends at a switch-box in an interconnect tile; the rest of the net's RouteTree continues on from this point. 


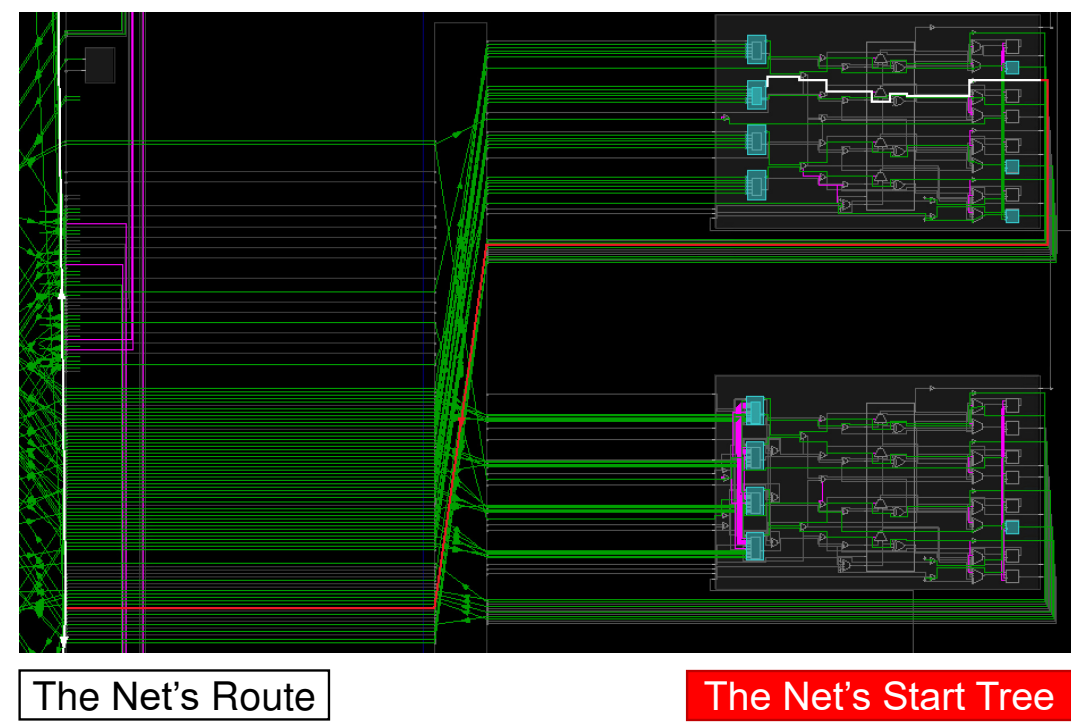

Figure 6.3: The Start Tree of an Inter-Site Route

With the created StartTrees, RSVRoute is able to begin routing nets from the last wire of their StartTrees instead of from their source site or partition pin. Because a net must utilize all routing resources within a StartTree to make a complete route, all of the routing resources within the StartTree become reserved for use by the corresponding net. This simplifies the later job of the $A^{*}$ maze router so it does not consider routing resources that are impossible for other nets to use.

The creation of the StartTree for static (VCC and GND) nets is a special case. This is because VCC and GND nets can (and usually do) have more than one driver. As shown in Figure 6.4, these nets are driven by tie-offs found within the device. However, the logical netlist typically only includes a single VCC net and a single GND net, with only one source cell pin each. This source cell pin belongs to a cell that cannot be physically placed on the device. This means that, unlike all other nets, the driving site output pins for the static nets are initially unknown to RSVRoute.

In nearly all cases, the sinks of static nets can be driven by a tie-off source in an adjacent interconnect tile, as seen in Figure 6.4. Therefore, RSVRoute identifies all such neighboring tieoffs for each static net. To minimize any special handling for static nets within PathFinder, a GlobalWire object is made for each static net. This GlobalWire represents a common physical source for a VCC or GND net. The VCC or GND GlobalWire alone makes up the StartTree 


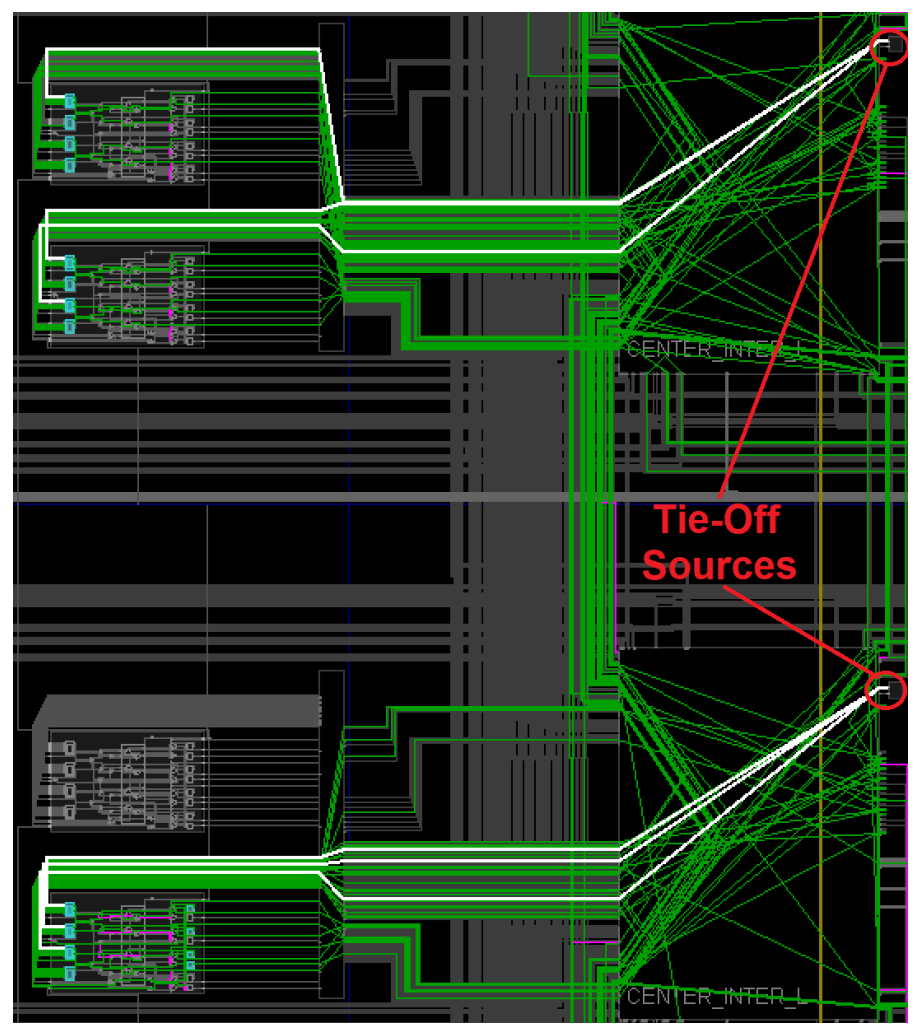

Figure 6.4: A Vivado VCC Net with Two Tie-Off Sources

for each static net. However, the identified tie-off sources are added as programmable connections that can be made from the GlobalWire, allowing VCC and GND to be routed as if they each only have one driver.

\section{Sink Trees}

Similar to the StartTrees, RSVRoute also creates one or more sink RouteTrees, henceforth referred to as SinkTrees, for each inter-site net. For each sink site pin or partition pin of a net, RSVRoute constructs SinkTrees by working backward. RSVRoute builds up the SinkTrees by connecting to PIPs in reverse, from sink wire to source wire, until a branch is first reached. Like the StartTrees, this allows RSVRoute to route to the first wire in each SinkTree instead of to the true terminal site pin or partition pin. All routing resources within the SinkTrees are also reserved for use by the corresponding net. 


\subsubsection{PathFinder}

Once IntersiteRoute objects have been created for each net, RSVRoute executes its implementation of PathFinder [39]. The traditional PathFinder algorithm consists of one or more routing iterations in which an internal path-finding algorithm routes all inter-site nets. Importantly, different inter-site nets are allowed to share routing resources to make up their routes. When a routing resource is being used by more than one route, it is considered to be congested. As long as there are congested resources, PathFinder continues to perform routing iterations in which all nets are ripped up and re-routed. However, as the algorithm continues, the cost of using the congested resources is increased. This, in effect, causes the inter-site nets to negotiate for the usage of routing resources. As congested routing resources become more expensive, nets are forced to use other routing resources instead. Eventually, all of the nets are routed with unique routing resources and PathFinder finishes execution.

The simplest version of PathFinder routes nets using a breadth-first maze routing algorithm, such as Djikstra's shortest-path algorithm [41]. The maze router applies a cost function to each node (routing resource) to increasingly minimize congestion over routing iterations. In a nontiming driven implementation of PathFinder, the cost function, $c_{n}$, applied to each node $n$ is

$$
c_{n}=\left(b_{n}+h_{n}\right) * p_{n}
$$

where $b_{n}$ is the base cost of using $n, h_{n}$ is the historical congestion penalty for using $n$, and $p_{n}$ is the present congestion penalty for using $n$. The $b_{n}$ term is typically set to the intrinsic delay of $n$. The $h_{n}$ term is based upon the over-use of $n$ during previous iterations and is needed to solve more complicated congestion problems. In contrast, the $p_{n}$ term is based only on the over-use of $n$ during the current routing iteration and is reset between iterations.

As suggested in [39], a directed maze routing algorithm, such as the A* best-first search algorithm [40], can also be used. In this case, the cost function must change. Specifically, when the $\mathrm{A}^{*}$ algorithm is used, as in RSVRoute, the cost of using node $n$ to route a net from source $i$ to sink $j$ becomes

$$
C_{n}=c_{n}+\text { Cest }_{i j}
$$


where Cest $_{i j}$ is the estimated cost (distance) of the route from $i$ to $j$.

\section{RSVRoute's PathFinder Implementation}

RSVRoute uses a modified non-timing driven implementation of PathFinder that optimizes wire-length. The pseudo-code for RSVRoute's implementation of PathFinder is given in Algorithm 1.

The main loop of the algorithm is entered on line 5; this loop exits once all the nets have been routed and no congestion exists. The nets themselves are actually routed within the inner loop of PathFinder. The inner loop of this algorithm will be discussed first, followed by an overview of the outer loop.

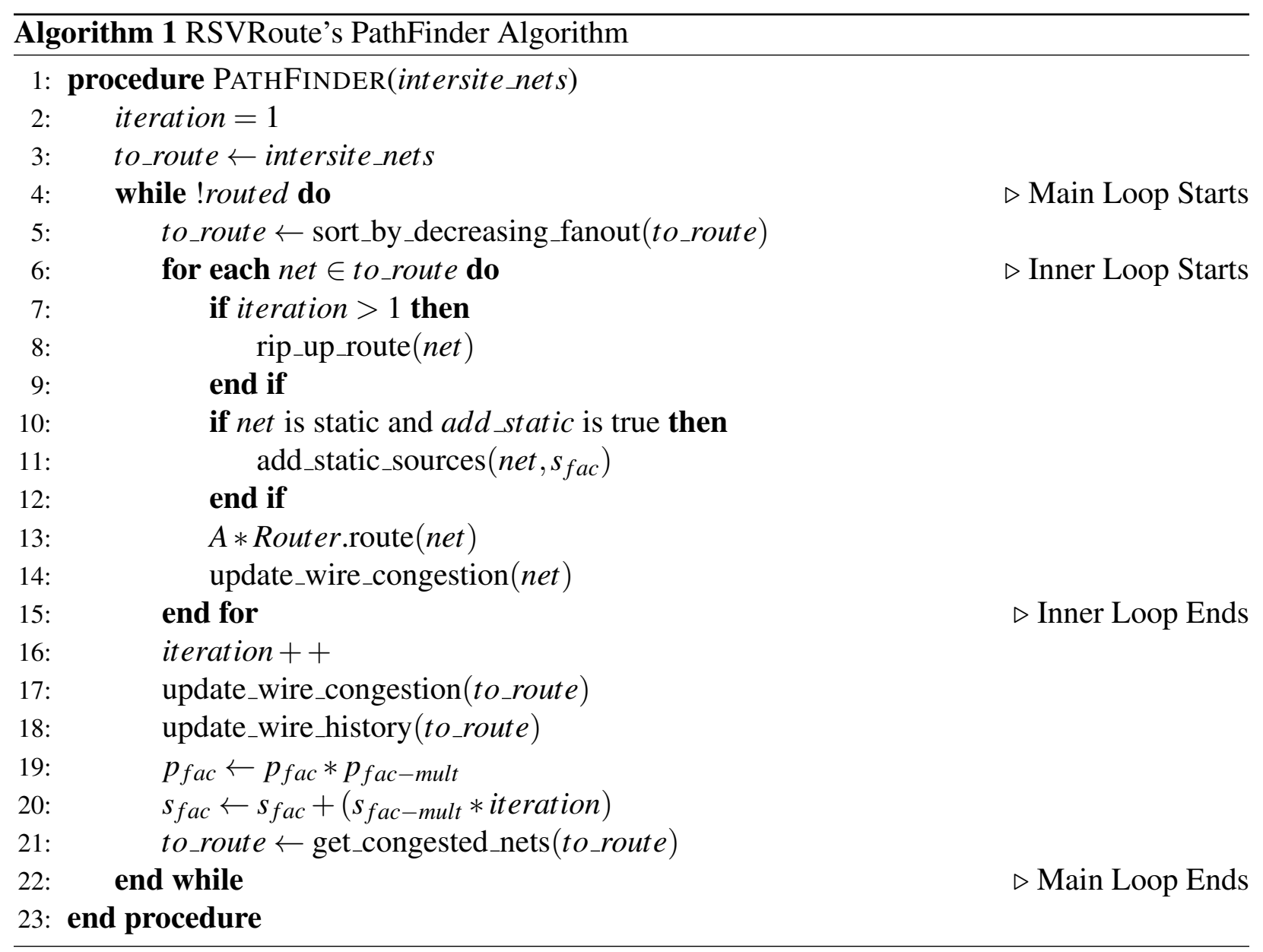


Inner Loop The main function of the inner loop is to route nets with the A* maze router. However, before each net is routed, a few steps are taken. First, as lines 7-9 of Algorithm 1 show, the previous routing of a net gets "ripped up". RSVRoute does this in such a way that the routes to a net's sinks which are not congested are preserved. As a result, the A* maze router only needs to re-route the sinks that were using congested routing resources.

If RSVRoute's add_static option is set, special additional steps are taken before static (VCC or GND) nets are routed. As mentioned earlier, RSVRoute routes nets starting StartTrees; in the case of static nets, this means the static nets are routed from special GlobalWires that can connect to all the tie-off sources neighboring the static sinks. In nearly all cases, all inter-site static sinks can be reached from these sources without causing irresolvable contention in a circuit's routing. However, in very rare circumstances, it is possible that another net may absolutely require the usage of specific routing resources that a static net would also require if it was driven by an interconnect tie-off.

To resolve this congested routing situation, it has been observed that Vivado will use a free LUT as a static source to drive the sink. An example of this situation is shown in Figure 6.5, with the static net highlighted in red and the net causing the congestion highlighted in white. RSVRoute models its handling of this routing situation after Vivado.

Because the number of LUTs in a device is huge, RSVRoute does not start off with all free LUTs added as connections to the static net's corresponding GlobalWire. Instead, if the add_static option is set, RSVRoute incrementally adds more free LUTs as programmable connections to the GlobalWire as PathFinder continues, dependent upon a static source factor, $s_{f a c}$. Specifically, for every un-routed static sink, RSVRoute searches for free LUTs $s_{f a c}$ tiles north, south, east, and west of the sink. Every found LUT is then added as a programmable connection to the GlobalWire, opening up new possible routes. The value of $s_{f a c}$ is updated in the main loop of PathFinder. However, this naive approach has been found to negatively impact run-time. Therefore, because this functionality is not needed in the vast majority of designs, RSVRoute currently disables it by setting the add_static option to false by default.

Following all this preparation, a net is finally routed with the A* maze router. RSVRoute uses the same cost function in equation $6.2, C_{n}=c_{n}+$ Cest $_{i j}$, for using a node $n$ to route a net from source $i$ to sink $j$. The estimated cost, or distance, Cest $t_{i j}$, of the path from node $i$ to $j$ is set to the 


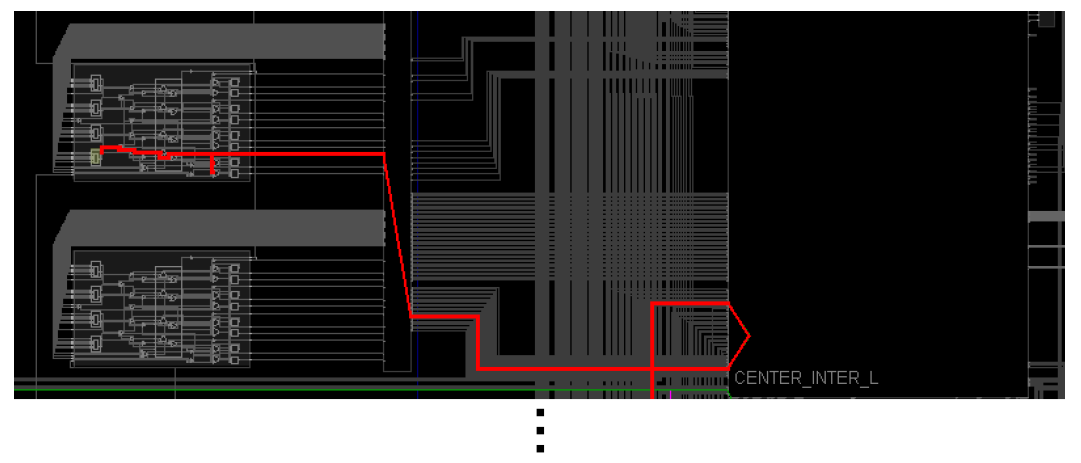

\section{In-between Tiles}

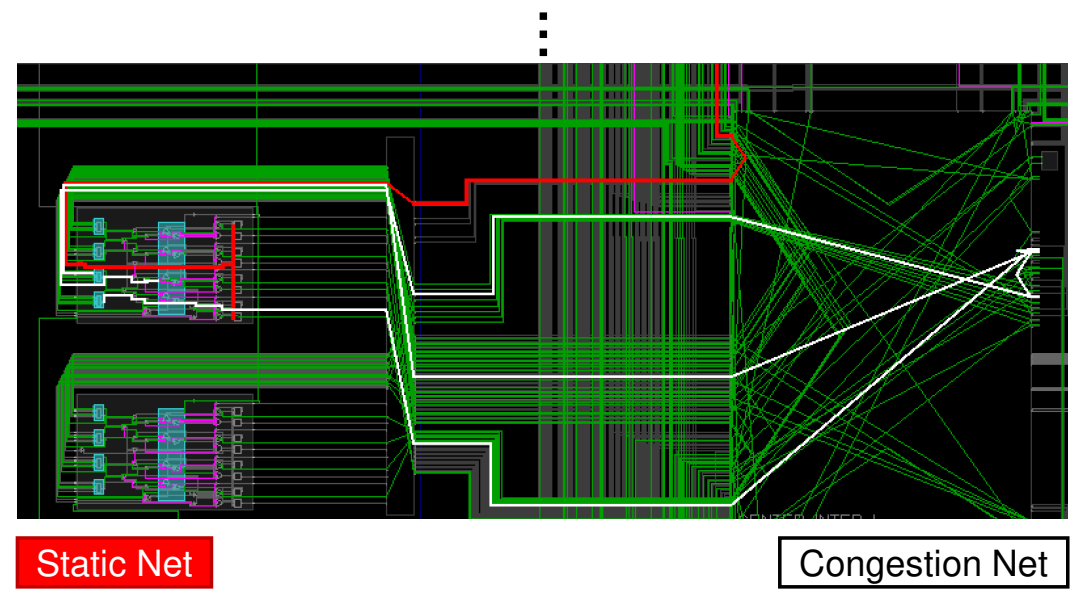

Figure 6.5: A LUT Static Source Used to Route a VCC Sink

quadratic distance from the source tile to the sink tile. The cost of a single node, $c_{n}$, is changed to

$$
c_{n}=b_{n} * h_{n} * p_{n}
$$

where the terms are all multiplied together as in Versatile Place and Route (VPR), [42] rather than adding $b_{n}$ and $h_{n}$. In RSVRoute, the base cost, $b_{n}$, of all nodes is set to 1 . The historical congestion penalty, $h_{n}$, used in RSVRoute is

$$
h_{n}= \begin{cases}1 & \text { iteration }=1 \\ 1+\max \left(0,\left(\text { occupancy }_{n}-1\right) * h_{f a c}\right) & \text { otherwise, }\end{cases}
$$

where occupancy $_{n}$ is the number of nets presently using node $n$ and $h_{f a c}$ is a historical scaling factor. While $h_{n}$ for every used node $n$ is updated in the outer loop, RSVRoute sets $h_{f a c}$ to a 
constant 1 by default, never increasing it. This behavior is modeled after VPR, but can easily be modified if desired.

RSVRoute calculates the present congestion penalty, $p_{n}$, as

$$
p_{n}=1+\max \left(0, \text { occupancy }_{n} * p_{\text {fac }}\right) \text {, }
$$

where occupancy $_{n}$ is as above and $p_{f a c}$ is a present congestion scaling factor. By default, RSVRoute initializes $p_{f a c}$ to 1 . Both $p_{n}$ and $p_{f} a c$ are updated in the outer loop of PathFinder. However, $p_{n}$ is also updated in the inner loop after each net is routed, as in VPR. By doing this, complex routing congestion problems can be solved and the overall execution time of PathFinder improves.

Several enhancements are used to improve the speed and quality of the results obtained by RSVRoute's A* maze router. Firstly, special care is taken for nets with more than one sink. Even though multi-sink nets share the same source, the A* algorithm must execute for each target sink. The order in which these sinks are routed can affect the total wire-length of the resulting route for the net. As shown in Figure 6.6a, routing the sinks by decreasing distance from the source results in the creation of long routing stems that are not well-reused. In contrast, routing the sinks by increasing distance from the source leads to further sinks re-using the routing resources used by earlier sinks, as shown in Figure 6.6b [6]. To further encourage the reuse of routing resources within a net, RSVRoute also makes the cost of using previously routed sinks free.

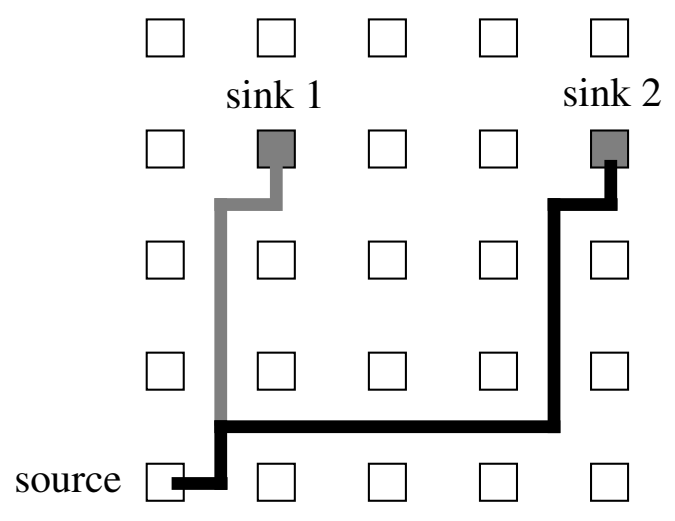

(a) Routing furthest sinks first.

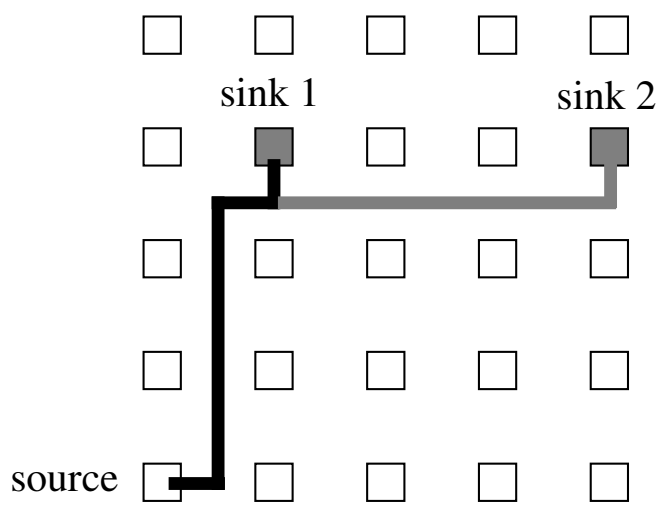

(b) Routing closest sinks first

Figure 6.6: Two Methods of Routing a Multi-Sink Net. Taken from [6]. 
RSVRoute's A* maze router also takes a number of steps to improve its execution speed. Firstly, the $\mathrm{A}^{*}$ maze router does not expand connections to routing resources that have been reserved for use by other nets—or by the static design. In addition, RSVRoute's A* maze router also takes the type of a net into consideration as it searches for a route. RSVRoute does not expand the connections to routing resources that a given net cannot utilize.

For instance, normal nets cannot use routing resources dedicated to clock nets. Additionally, global clock buffer nets and local clock nets also differ in the types of routing resources they can use, as shown in Figure 6.7. Global clock buffer nets can utilize special dedicated routing resources, such as CLK, HCLK, CLK_HROW, and CLK_BUFG wires, to make it easier for designs to meet timing. In contrast, local clock nets utilize local routing resources to make their routes.

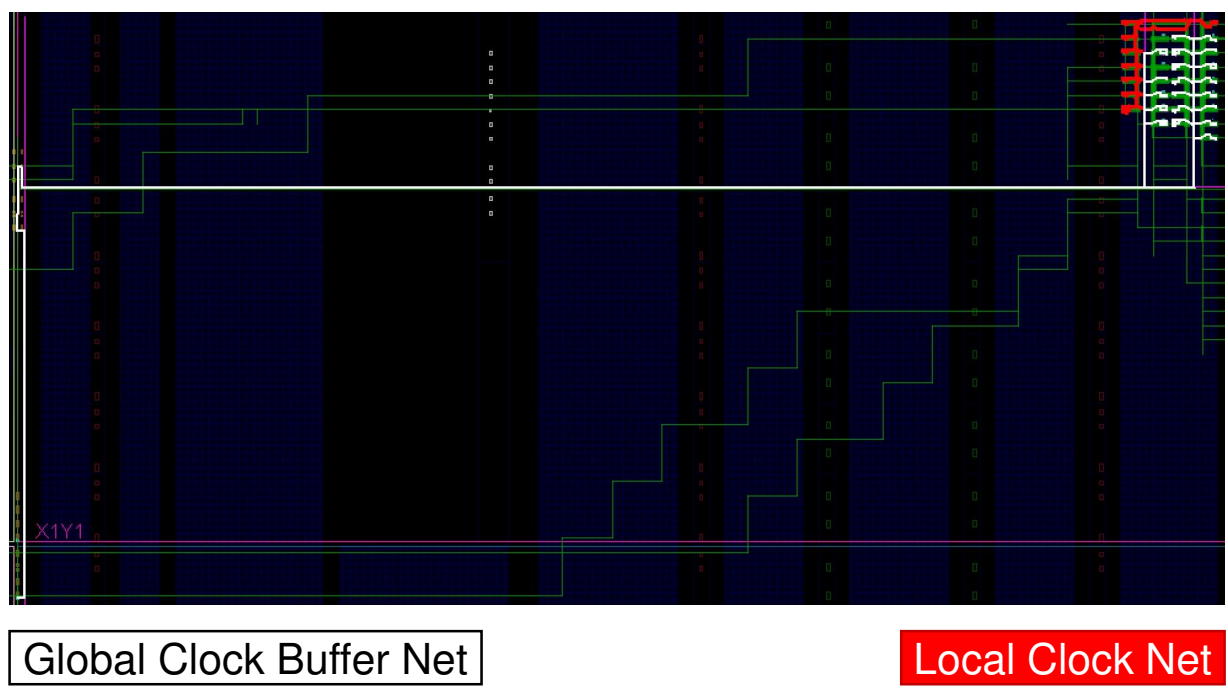

Figure 6.7: A Routed Global Clock Buffer Net and a Routed Local Clock Net

Outer Loop Because the congestion of nodes is updated in the inner loop after each net is routed, it is easier (and faster) to route nets that come first in a routing iteration. Therefore, as line 5 of Algorithm 1 shows, the outer loop executes Quicksort [43] to sort the inter-site nets in order of decreasing fan-out before every iteration of the inner loop. The nets are routed in this order because high fan-out nets tend to span more of the FPGA, while low fan-out nets tend to be more localized. The high fan-out nets are easier to route when the costs of using congested nodes is cheaper [6]. 
As previously mentioned, $p_{n}$ is updated again in the outer loop, using Equation 6.5. As in the traditional version of PathFinder, $h_{n}$ is updated only in the outer loop with Equation 6.4. Line 19 of Algorithm 1 shows that $p_{f a c}$ is multiplied by $p_{f a c-m u l t}$ after every routing iteration [42]. By default, RSVRoute sets $p_{\text {fac-mult }}$ to 1 . Additionally, line 20 shows that the static source factor, $s_{f a c}$, is increased by the product of the iteration number and $s_{f a c-m u l t}$, a value which can be set by the user.

Finally, the outer loop updates the list of nets that need to be routed, to_route, to only include nets that are using presently congested routing resources. By doing this, only these nets are re-routed in the next iteration of pathfinder. This can cause more routing iterations to be required to successfully route a circuit, but each iteration is faster, resulting in an overall faster run-time [39].

\subsubsection{Limitations}

As RSVRoute is a relatively new router that was created mainly to accomplish the goals of Maverick, it has some limitations. The run-time and quality of results, which can be improved, will be given and analyzed in Section 7.1. Two other current limitations of RSVRoute are discussed here.

Firstly, as mentioned, the current approach to adding unoccupied LUTs as extra static sources negatively impacts run-time. Because additional static sources don't need to be added for almost all designs, it is disabled by default. Alternative methods could be investigated in the future to make this feature more efficient.

Additionally, RSVRoute currently does not route to BRAM or DSP primitives. The decision to not support these cells was made for two reasons. First, the version of Project X-Ray used by Maverick did not support these device primitives, making support unimportant for the goals of this work. Second, the presence of several polarity muxes on BRAM and DSP primitives currently complicates routing in RapidSmith2 as RapidSmith2's device representation does not include polarity muxes. Once proper support for polarity muxes is added to RapidSmith2, support BRAMs and DSPs can easily be added to RSVRoute. 


\subsubsection{Partial Reconfiguration (PR) Features}

As mentioned, RSVRoute was created with the goals of Maverick in mind. Because of this, it can route RM designs, contained in a PR region, in addition to full-device designs. RSVRoute has two essential features which enable this functionality.

First, RSVRoute's A* maze router does not allow nets to use reserved routing resources, including resources used by the static design. This functionality was largely motivated by the need to ensure that the RM does not create routes that conflict with the static design's routes. The second essential feature is that RSVRoute does not route only from output site pins to input site pins, but also between partition pins and site pins. As partition pins are wire segments in the routing interconnect, this really means that RSVRoute can route between arbitrary wires. Without these features, Maverick would be unable to route its RM designs.

\subsection{FASM Generation: RapidSmith2}

Once the routing is complete, the design is fully physically implemented, and Maverick only needs to generate a final partial bitstream. As shown in Figure 6.1, this is accomplished through the use of both RapidSmith2 and Project X-Ray tools. The first step is the generation of an FPGA Assembly (FASM) file. Once a FASM file has been generated, Project X-Ray tools are used to patch the initial partial bitstream generated in the static design phase.

As explained in Section 3.4, a FASM file contains instructions that enable features of specific FPGA resources. Each instruction specifies a bit in the initial partial bitstream that needs to either be set (1) or cleared (0). The omission of an instruction for any given feature simply means that the default state of the feature, as reflected in the initial partial bitstream, is unchanged.

A FASM Generator has been added to RapidSmith2 to enable the creation of FASM files for routed RM designs. This section presents the steps that this generator takes to produce FASM instructions for the device features supported by Maverick. 


\subsubsection{Logic BEL Instructions}

The first type of instructions the FASM generator must produce instructs the FPGA on how to configure logic BELs within the FPGA. For Maverick, this includes instructions for LUT Bels and flip-flop (FF) BELs.

\section{LUT BELS}

The FASM instructions for a LUT BEL depend upon the type of cell that is placed upon the BEL. In Maverick, this may be any type of LUT cell or a RAMD64E LUT RAM cell. The instructions for both cases will be discussed in this respective order.

LUT Cells LUT BELs occupied by LUT cells are configured with one or more INIT instructions, which together correspond to a LUT's truth table. Each input into the truth table is one of the physical LUT BEL pins (A1 - A6). Since the Xilinx 7-Series FPGAs use fracturable 6-input LUTs (LUT6 BELs), an INIT value representing a LUT's truth table is $64\left(2^{6}\right)$ bits wide. Accordingly, each individual INIT instruction for a LUT BEL configures one of these bits. Table 6.1 shows an abbreviated truth table for the LUT equation

$$
O 6=(A 1 *(\sim A 2) * A 3 *(\sim A 4))+((\sim A 1) * A 2 * A 3 *(\sim A 4)) .
$$

Table 6.1: Truth Table for Equation 6.6. Rows with O6 = 0 are Omitted.

\begin{tabular}{|c|c|c|c|c|c|c|c|}
\hline & A6 & A5 & A4 & A3 & A2 & A1 & O6 \\
\hline $\mathbf{5}$ & 0 & 0 & 0 & 1 & 0 & 1 & 1 \\
$\mathbf{6}$ & 0 & 0 & 0 & 1 & 1 & 0 & 1 \\
$\mathbf{2 1}$ & 0 & 1 & 0 & 1 & 0 & 1 & 1 \\
$\mathbf{2 2}$ & 0 & 1 & 0 & 1 & 1 & 0 & 1 \\
$\mathbf{3 7}$ & 1 & 0 & 0 & 1 & 0 & 1 & 1 \\
$\mathbf{3 8}$ & 1 & 0 & 0 & 1 & 1 & 0 & 1 \\
$\mathbf{5 3}$ & 1 & 1 & 0 & 1 & 0 & 1 & 1 \\
$\mathbf{5 4}$ & 1 & 1 & 0 & 1 & 1 & 0 & 1 \\
\hline
\end{tabular}


Listing 6.1 shows the instructions used to configure the BLUT in SLICE_X65Y28, which is at slice index 1 of the CLBLL_L_X42Y28 tile, with this same LUT equation. As can be seen, a FASM instruction is needed for each row of the truth table in which $\mathrm{O} 6=1$.

Listing 6.1: FASM Instructions for Equation 6.6

CLBLL_L_X42Y28.SLICEL_X1.BLUT. INIT[05] 1
CLBLL_L_X42Y28.SLICEL_X1.BLUT. INIT[06] 1
CLBLL_L_X42Y28.SLICEL_X1. BLUT. INIT [21] 1
CLBLL_L_X42Y28.SLICEL_X1.BLUT. INIT[22] 1
CLBLL_L_X42Y28.SLICEL_X1. BLUT. INIT[37] 1
CLBLL_L_X42Y28.SLICEL_X1. BLUT. INIT[38] 1
CLBLL_L_X42Y28.SLICEL_X1. BLUT. INIT[53] 1
CLBLL_L_X42Y28.SLICEL_X1. BLUT. INIT[54] 1

The RapidSmith2 FASM generator must take a number of factors, given below, into consideration to produce INIT instructions for a given LUT BEL occupied by a LUT cell.

- The INIT instructions must reflect the physical truth table of the LUT, not the logical truth table. All LUT cells in an EDIF netlist have an INIT property that represents a LUT's truth table in terms of logical LUT cell pins (I0 - I5) and not in terms of physical LUT BEL pins (A1 - A6). By default, the logical pins are mapped to the physical pins in a straightforward manner, with I0 mapping to A1, I1 mapping to A2, etc. However, the pin mappings are often rearranged to assist with routing congestion in a design. For instance, A1 may be changed to map to I3 instead of I0, and A2 may be changed to map to I0 instead. Because of this, the FASM generator reformulates all LUT equations from the EDIF netlist to account for different pin mappings.

- The INIT properties in the EDIF netlist are only in terms of the inputs that are actually used. However, the INIT instructions must account for all physical pins connected to the LUT, even unused ones. Therefore, if the LUT BEL is a 5-input LUT (LUT5), the LUT equation must be reformulated to account for 5 inputs. And if the LUT BEL is a 6-input LUT (LUT6), it must be reformulated to account for 6 inputs. 
- The INIT instructions are further affected by the use of fracturable LUTs in Xilinx 7-Series FPGAs. The INIT values of a LUT6 BEL and a LUT5 BEL combine to create a 64-bit INIT value, meaning they share one set of FASM INIT instructions. Accordingly, if a LUT6 BEL is occupied by a LUT6 cell or if the corresponding LUT5 BEL is unoccupied, the INIT value for the LUT6 BEL must 64 bits wide. However, if both the LUT6 BEL and LUT5 BEL are occupied, the most-significant 32 bits of the combined INIT value correspond to the LUT6, while the least-significant 32 bits correspond to the LUT5's INIT value.

LUT RAM Cells LUT RAM macro cells are made up of relatively placed internal cells. The LUT BELs occupied by the LUT RAM cells are configured with INIT instruction as well as RAM instructions. These instructions depend not only upon the RAM initialization contents, but also on the specific type of the LUT RAM. As discussed in Section 3.1, Yosys does not support all possible configurations of LUT RAMs. Because of this, the FASM generator currently only support LUT RAMs that Yosys supports; however, this could be expanded in the future. At this time, the FASM generator supports the RAM64X1D and RAM128X1D LUT RAM macros, which are both internally composed of RAMD64E cells.

Each individual LUT BEL in a LUT RAM gets its own set of FASM instructions. The simplest instruction is the RAM instruction, which denotes that a LUT BEL is being used as a RAM. For example, “CLBLM_R_X43Y38.SLICEM_X0.CLUT.RAM 1" instructs that the CLUT of SLICE_X66Y38, which is at index 0 of CLBLM_R_X43Y38, is being used as a RAM.

INIT instructions must also be generated for each LUT BEL used as a LUT RAM. Like LUT cells, LUT RAM macro cells include INIT properties in the EDIF netlist. Also, like LUT cells, an individual INIT instruction must be written for every set bit of the BEL's INIT value. However, since LUT RAMs hold memory, the INIT property should not be viewed as a truth table implementing a logic equation, but instead as the initialization contents of a RAM.

However, because the EDIF netlist gives the INIT property for the entire LUT RAM cell, the INIT value and instructions for the individual BELs is not immediately available. The INIT value for the individual BELs depends upon the type of the macro cell, as explained below. 
- RAM64X1D macro cells are composed of two RAMD64E cells, with one named SP and one named DP. The INIT value for both of the BELs occupied by the SP and DP cells is equal to the macro's INIT property given in the EDIF netlist.

- RAM128X1D macro cells contain four RAMD64E cells, named DP.HIGH, DP.LOW, SP.HIGH, and SP.LOW. The INIT values for the BELs occupied by the DP.LOW and SP.LOW cells are equal to the most-significant 64 bits of the macro cell's INIT property. The INIT values for the DP.HIGH and SP.HIGH BELs are equal to the least-significant 64 bits of the macro cell's INIT property.

\section{Flip-flop BELs}

There are only two types of FASM instructions for individual FF BELs: ZINI and ZRST instructions. The ZINI instructions set the global-set-reset (GSR) value for the BEL, while the ZRST instructions set the value for the BEL upon a reset. Like LUT BELs, these instructions depend upon the type of the occupying cell, which may be either a FF (FDPE, FDSE, FDRE, FDCE) or latch (LDCE, LDPE) cell.

Both FF and latch cells have INIT properties in the EDIF netlist that are set to either 0 or 1. If a BEL is occupied by a FF cell, this value must be inverted to obtain the BEL's ZINI instruction. For instance, if a FF cell placed on SLICE_X66Y36 has an INIT property of 0, the FASM instruction "CLBLM_R_X43Y36.SLICEM_XO.AFF.ZINI 1" must be included in the FASM file. No instruction is needed if a FF cell has an INIT property of 1 . In contrast, if a BEL is instead occupied by a latch cell, the INIT property from the netlist is not inverted. For example, if a latch cell placed on SLICE_X62Y34 has an INIT property of 1, the corresponding FASM instruction would be "CLBLM_R_X41Y34.SLICEM_X0.AFF.ZINI 1". No instruction is needed if a latch cell has an INIT property of 0.

The ZRST instruction only depends upon the specific type of cell that occupies the FF BEL. If the BEL is occupied by an FDPE, FDSE, or LDPE cell, an instruction must be written. For instance, the instruction "CLBLM_R_X43Y37.SLICEM_X0.BFF.ZRST 1" must be written if the BFF BEL in SLICE_X66Y37 is occupied by any of the previously mentioned types of cells. If the BEL is occupied by an FDRE, FDCE, or LDCE cell, no instruction is needed. 


\subsubsection{Site-Wide Instructions}

The second type of FASM instructions are site-wide instructions. This includes instructions to enable features that all BELs within a given site share as well as instructions for configuring site PIPs.

\section{Shared Features}

Shared features of a site, sometimes referred to as a site's control set, are features that all BELs within a site share; it is not legal for these features to differ between the BELs. There are three types of features that the FASM Generator produces instructions for.

- CLKINV: controls whether or not the clock signal driving a slice should be inverted with the slice's CLKINV polarity mux. Because RapidSmith2's device representation does not include polarity muxes [44], the FASM generator uses the IS_C_INVERTED property of FF cells in the netlist to determine if the clock should be inverted. If a slice contains FF cells, the CLKINV feature gets set. Otherwise, it is cleared.

- FFSYNC: controls whether all the FFs in a slice use a synchronous or asynchronous reset. If a slice contains FDSE or FDRE cells, the slice must use an asynchronous reset; other types of FF/Latch cells require a synchronous reset. If a slice contains FFs, this feature should be set (1) for an asynchronous reset to be used and should be cleared (0) for the FFs to use a synchronous reset. The reverse is true if a slice contains latches; this feature should be set for a synchronous reset and cleared for an asynchronous reset.

- LATCH: specifies that all FF BELs are being used as FFs or latches. This feature is set if latches are being used and is cleared if FFs are being used.

\section{Site PIPs}

An instruction must also be written for each routing mux that is used within a site. For instance, the instruction "CLBLM_R_X39Y18.SLICEM_X0.DFFMUX DX 1" configures the DFFMUX of SLICE_X58Y18 to use the DX input. It is relatively straightforward for the RapidSmith2 FASM generator to configure a site's internal PIPs. After the completion of RSVPack and RSVPlace, 
Maverick saves the configuration of every routing mux to an internal data structure within RapidSmith2. Therefore, the FASM generator simply traverses this data structure and writes out the appropriate instructions.

\subsubsection{Inter-Site Routing Instructions}

The third type of instructions the FASM generator creates are inter-site routing instructions. Specifically, these instructions turn on PIPs in the FPGA's routing interconnect. For example, the instruction "INT_L_X38Y16.SE2BEG1 LOGIC_OUTS_L1" turns on the PIP in tile INT_L_X38Y16 with start wire LOGIC_OUTS_L1 and end wire SEG2BEG1.

To generate the instructions, the FASM generator traverses each net's RouteTree that was created by RSVRoute. Each time a PIP is encountered in the tree, a corresponding instruction is written. No instructions are written for non-programmable connections in the route tree.

The FASM generator also does not output instructions for "pseudo-pips". Pseudo pips are connections in the FPGA that appear as PIPs in both Vivado and RapidSmith2, but actually have no corresponding bit pattern in the bitstream [3]. Project X-Ray can be used to document all of the pseudo-pips in a Xilinx 7-Series FPGA. Currently, two types of pseudo PIPs are of concern for the FASM generator.

- Always-on: PIPs that are always enabled. These PIPs include all PIPs in CLB tiles, as well as a number of PIPs in the interconnect tiles' switch-boxes.

- Default: PIPs that are used by default if no other net is driving the PIP's end wire. All default PIPs are in interconnect switch-boxes. By default, they are driven by the VCC tie-off wire located in the same tile.

In addition to turning on PIPs, the FASM generator must also write instructions to enable the HCLK buffers used by global clock buffer nets. For instance, the instruction "HCLK_R_X109Y26.ENABLE_BUFFER.HCLK_CK_BUFHCLKO 1" enables HCLK_CK_BUFHCLKO within tile HCLK_R_X109Y26. 


\subsubsection{Static Design Instructions}

Lastly, the FASM generator writes instructions for routing resources within the PR region that are used by the static design. This includes instructions for any routing interconnect PIPs used by the static design as well as any site PIPs and LUT route-throughs within sites used as site routethroughs by the static design. To enable the routing interconnect PIPs, the FASM generator simply traverses the RouteStringTrees for the static-design nets, similar to how the FASM generator traverses standard Routrees. The FASM generator uses the lists of site PIPs and LUT routethroughs that are imported upon RM RSCP import, as described in Subsection 4.2.3, in order to correctly configure the site route-throughs. The instructions for both static site PIPs and LUT route-throughs are formatted in the same way as they are in the RM design.

\subsection{Bitstream Generation: Project X-Ray}

Finally, once all the FASM instructions are generated, Maverick leaves RapidSmith2 and uses Project X-Ray tools to generate the new partial bitstream, as shown in Figure 6.1. To do so, Maverick uses the newly generated FASM file as well as the Project X-Ray bitstream database and initial partial bitstream generated in the static design phase.

In the first part of this final step, Project X-Ray's existing FASM2FRAME Python program uses the bitstream database to convert the FASM file to a FRM file. This FRM file is simply a text file that contains all the configuration frames for the target PR region. The FASM2FRAME program correctly calculates the ECC value for each frame as well, including it in the 50th word of each frame.

Next, the xc7PartialPatch program, created for Maverick, patches the initial partial bitstream with the contents of the FRM file. This program is based on Project X-Ray's existing xc7Patch program but was modified to create partial bitstreams instead of full-device bitstreams, using the understanding of partial bitstreams described in Subsection 2.3.1. The partial bitstream generated by xc7PartialPatch is organized as shown in Figure 6.8, reproduced from Figure 2.14a.

Specifically, xc7PartialPatch creates a new header for the partial bitstream, including the expected information. Then, xc7PartialPatch inserts the CLB_CFG frames section from the initial partial bitstream. The configuration frames from the FRM file are inserted into the bitstream next, 


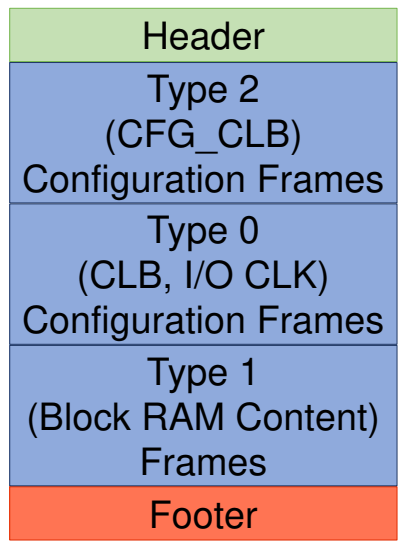

Figure 6.8: Organization of Partial Bitstreams Produced by xc7PartialPatch

and a footer is lastly created and added to the bitstream. Note that blanking frames are currently not included in the partial bitstream by xc7PartialPatch. This resulting partial bitstream can then be configured onto the FPGA once it has first been programmed with a full-device bitstream. 


\section{CHAPTER 7. RESULTS AND EXPERIMENTS}

Several tests and experiments were performed to evaluate the quality and performance of this work. This chapter presents and analyzes the results of these tests. All of the tests and experiments discussed in this chapter target the Xilinx 7-Series xc7z020clg400-1 Zynq device, which Digilent's PYNQ-Z1 board contains.

Section 7.1 first discusses testing that was done to evaluate RSVRoute in isolation from the rest of Maverick. The run-time and resulting quality of routing several full-device benchmarks with both Vivado and RSVRoute are presented and analyzed. Next, Section 7.2 gives results on Maverick as a whole. Relevant results of the static design phase are presented and the results of compiling a set of RM designs with Maverick's RM phase are then given. Finally, Section 7.3 describes the results of executing Maverick's RM phase on the embedded PYNQ-Z1 board's ARM CPU. An experiment in which Maverick was more tightly integrated into Xilinx's PYNQ system is also described. In this experiment, a set RM designs compiled with Maverick were programmed onto the PYNQ-Z1's FPGA and were shown to function correctly.

\subsection{RSVRoute Results}

An important result of this work is RSVRoute, which was created to route RM designs in Maverick. However, as explained in Section 6.5, RSVRoute can also route full-device designs. Therefore, this section evaluates RSVRoute in isolation to investigate opportunities for future work. The tests described in this section were executed on a desktop computer with an Intel i7 $860 \mathrm{CPU}$, which runs at $2.80 \mathrm{GHz}$, and $12 \mathrm{~GB}$ of RAM.

In order to test RSVRoute, several full-device benchmark designs of various sizes originating from OpenCores [45] and Yosys [46] were synthesized and then placed with Vivado. Because RSVRoute currently does not support routing to BRAMs or DSPs, synthesis was performed using [synth_design -max_bram 0 -max_dsp 0]. Placement was run using the place_design 
command and no additional parameters. Table 7.1 gives the resulting netlist and resource usage information for each of these designs.

Table 7.1: RSVRoute Benchmarks

\begin{tabular}{|l|r|r|r|r|}
\hline Benchmark & \# Cells & \# Nets & Slice Util. & IOB Util. \\
\hline regfile & 87 & 59 & $0.05 \%$ & $9.34 \%$ \\
crc32 & 160 & 128 & $0.08 \%$ & $12.65 \%$ \\
transmitter & 195 & 177 & $0.14 \%$ & $8.73 \%$ \\
sddac & 158 & 221 & $0.11 \%$ & $5.72 \%$ \\
stopwatch & 246 & 242 & $0.23 \%$ & $7.53 \%$ \\
pattern_game & 355 & 370 & $0.34 \%$ & $8.43 \%$ \\
cordic_10_16 & 344 & 374 & $0.11 \%$ & $34.34 \%$ \\
timergame & 417 & 461 & $0.38 \%$ & $7.83 \%$ \\
picorv32_small & 1581 & 1779 & $1.64 \%$ & $31.63 \%$ \\
lu8peeng & 1772 & 1880 & $1.97 \%$ & $3.01 \%$ \\
dspmac_24_56 & 1375 & 1958 & $1.54 \%$ & $32.53 \%$ \\
des & 3099 & 3059 & $3.05 \%$ & $0.60 \%$ \\
fastfir_fixedtaps & 3211 & 3304 & $3.76 \%$ & $10.24 \%$ \\
aquarius & 9397 & 11281 & $17.86 \%$ & $10.54 \%$ \\
ecg & 18727 & 18427 & $18.80 \%$ & $1.51 \%$ \\
canny & 13628 & 19816 & $28.70 \%$ & $6.02 \%$ \\
ucsb_152_tap_fir & 28171 & 33083 & $20.02 \%$ & $12.65 \%$ \\
viterbi7 & 55252 & 55429 & $98.92 \%$ & $2.11 \%$ \\
\hline
\end{tabular}

The results of routing the designs with both Vivado and RSVRoute are given in Tables 7.2 and 7.3 below. Table 7.2 gives the run-times and Table 7.3 gives the resulting wire-length, measured in terms of tiles traversed. Routing was considered to have failed for designs that did not finish routing in under 35 minutes. To route these designs with Vivado, the route_design command was used with no additional parameters and no timing constraints; this causes Vivado's router to optimize only for wire-length and routing congestion [47]. This was done to provide a more accurate comparison against RSVRoute.

RSVRoute itself was executed 4 times with different parameters values, which are described in Section 6.5.2. The parameters that mainly affect the run-time and quality of the results are $p_{f a c}, p_{f a c-m u l t}$, and $h_{f a c}$. While a full parameter sweep is not given here, Table 7.2 and 
Table 7.2: Comparison of Routing Run-times (in Seconds) ${ }^{1}$

\begin{tabular}{|l|r|r|r|r|r|r|}
\hline \multicolumn{1}{|c|}{ Benchmark } & \# Nets & Vivado & $\begin{array}{c}\text { RSVRoute } \\
\mathbf{( 1 , 1 . 3 , \mathbf { 1 } )}\end{array}$ & $\begin{array}{c}\text { RSVRoute } \\
\mathbf{( 1 0 , 1 . 3 , 1 0 )}\end{array}$ & $\begin{array}{c}\text { RSVRoute } \\
(\mathbf{1 0 0 , 1 . 3 , 1 0 0 )})\end{array}$ & $\begin{array}{c}\text { RSVRoute } \\
(\mathbf{1 0 0 0 , 1 . 3 , ~ 1 0 0 0 )})\end{array}$ \\
\hline regfile & 59 & $8.00^{2}$ & 0.42 & 0.34 & 0.27 & 0.26 \\
crc32 & 128 & 8.00 & 0.78 & 0.52 & 0.56 & 0.54 \\
transmitter & 177 & 8.00 & 0.90 & 0.58 & 0.74 & 0.55 \\
sddac & 221 & 14.00 & 2.17 & 1.80 & 0.32 & 0.33 \\
stopwatch & 242 & 8.00 & 0.70 & 0.56 & 0.59 & 0.56 \\
pattern_game & 370 & 8.00 & 1.01 & 0.71 & 0.77 & 0.71 \\
cordic_10_16 & 374 & 8.00 & 1.27 & 0.93 & 0.90 & 1.04 \\
timergame & 461 & 8.00 & 1.15 & 0.87 & 0.93 & 1.02 \\
picorv32_small & 1779 & 9.00 & 18.62 & 19.04 & -3 & - \\
lu8peeng & 1880 & 9.00 & 10.36 & 5.26 & 5.32 & 5.47 \\
dspmac_24_56 & 1958 & 21.00 & 12.59 & 6.09 & 6.38 & 6.15 \\
des & 3059 & 9.00 & 18.80 & 11.08 & 85.11 & 511.59 \\
fastfir_fixedtaps & 3304 & 10.00 & 11.57 & 6.93 & 6.38 & 6.86 \\
aquarius & 11281 & 55.00 & 1624.53 & - & - & - \\
ecg & 18427 & 16.00 & 233.59 & 175.56 & - & - \\
canny & 19816 & 52.00 & 344.47 & 174.29 & - & - \\
ucsb_152_tap_fir & 33083 & 43.00 & 73.00 & 44.65 & 321.05 & - \\
viterbi7 & 55429 & 74.00 & - & - & - & -18 \\
\hline
\end{tabular}

${ }^{1}$ Each RSVRoute column gives the results of runs with different values for $p_{f a c}, p_{m u l t-f a c}$, and $h_{f a c}$, respectively.

${ }^{2}$ Vivado reports routing run-time only down to seconds, not milliseconds.

${ }^{3} \mathrm{~A}$ " - " indicates that the design failed to route in under 35 minutes.

Table 7.3 include results for 4 different runs of RSVRoute using different parameter values for each design.

The run-times in Table 7.2 reveal some interesting insights into the performance of RSVRoute.

Firstly, RSVRoute is actually able to route the smallest designs more quickly than Vivado, which reported a minimum of 8 seconds for the smallest designs. However, RSVRoute quickly becomes slower than Vivado when even slightly larger designs, such as picorv32_small, are routed.

The values of RSVRoute's parameters have an obvious effect on its performance. These parameters determine how costly it is for a net to use congested routing resources. In theory, higher values for these parameters should cause the router to complete more quickly as the router should avoid using the congested resources which have been made very expensive to use. Essentially, the router should trade run-time for some decrease in the quality of its results. ${ }^{1}$ As expected,

\footnotetext{
${ }^{1}$ This outcome is seen in VPR's fast routing schedule, in which the routing can complete two to three times faster than normal [42].
} 
Table 7.3: Routing Wire-length Comparison (in Tiles Traversed) ${ }^{1}$

\begin{tabular}{|l|r|r|r|r|r|r|}
\hline \multicolumn{1}{|c|}{ Benchmark } & \# Nets & Vivado & $\begin{array}{c}\text { RSVRoute } \\
\mathbf{( 1 , 1 . 3 , \mathbf { 1 } )}\end{array}$ & $\begin{array}{c}\text { RSVRoute } \\
\mathbf{( 1 0 , 1 . 3 , ~ 1 0 )}\end{array}$ & $\begin{array}{c}\text { RSVRoute } \\
(\mathbf{1 0 0 , 1 . 3 , 1 0 0 )})\end{array}$ & $\begin{array}{r}\text { RSVRoute } \\
(\mathbf{1 0 0 0 , 1 . 3 , ~ 1 0 0 0 )})\end{array}$ \\
\hline regfile & 59 & 518 & 827 & 830 & 529 & 529 \\
crc32 & 128 & 3371 & 3747 & 3786 & 3786 & 3786 \\
transmitter & 177 & 835 & 943 & 1039 & 1039 & 1039 \\
sddac & 221 & 698 & 830 & 853 & 853 & 853 \\
stopwatch & 242 & 1113 & 1093 & 1131 & 1131 & 1131 \\
pattern_game & 370 & 1535 & 1444 & 1502 & 1502 & 1502 \\
cordic_10_16 & 374 & 9277 & 9647 & 9607 & 9607 & 9607 \\
timergame & 461 & 2021 & 1951 & 2091 & 2091 & 2091 \\
picorv32_small & 1779 & 28958 & 33386 & 37545 & -2 & - \\
lu8peeng & 1880 & 12675 & 14557 & 16480 & 16534 & 16534 \\
dspmac_24_56 & 1958 & 24078 & 26917 & 28500 & 28510 & 28510 \\
des & 3059 & 24730 & 36110 & 41370 & 41674 & 41671 \\
fastfir_fixedtaps & 3304 & 19295 & 15323 & 15780 & 15780 & 15780 \\
aquarius & 11281 & 251600 & 289770 & - & - & - \\
ecg & 18427 & 189415 & 234600 & 283293 & - & - \\
canny & 19816 & 407306 & 414714 & 445722 & - & -193137 \\
ucsb_152_tap_fir & 33083 & 173869 & 180147 & 193086 & 193137 & - \\
viterbi7 & 55429 & 773556 & - & - & - & - \\
\hline
\end{tabular}

${ }^{1}$ Each RSVRoute column gives the results of runs with different values for $p_{f a c}, p_{m u l t-f a c}$, and $h_{f a c}$, respectively.

${ }^{2}$ A “-” indicates that the design failed to route in under 35 minutes.

RSVRoute completes more quickly when the values are initially increased from $1,1.3$, and 1 to $10,1.3$, and 10 , for $p_{f a c}, p_{f a c-m u l t}$, and $h_{f a c}$, respectively. However, further increasing the parameter values instead slowed the router down-extremely in some cases. The most likely cause of this result is the use of less efficient data structures in RSVRoute and RapidSmith2. RSVRoute must keep track of all wires it has ever encountered and it also keeps a priority queue of partially built routes as it creates routes for each net. If a net must use a specific wire that has been made extremely expensive since another net has already used it, RSVRoute will continue to add to these data structures until it finally determines that the expensive wire must be used. As these data structures grow to large sizes, RSVRoute's performance suffers. In more congested routing scenarios, such as in the viterbi7 design, this can cause RSVRoute to fail entirely in that the required time to completely route the design becomes unacceptably long.

Furthermore, the results in Table 7.3 show that Vivado was able to create routed designs of a higher quality than RSVRoute, since the total number of tiles traversed by Vivado's routes was 
always less than that of RSVRoute. This in unsurprising, but RSVRoute is still able to achieve competitive results on a fair number of designs. Additionally, it can be seen that the total wirelengths generally increased up to a point as the parameter values of RSVRoute were increased. This is expected as RSVRoute should be more willing to accept less efficient routes to more quickly complete with higher parameter values.

These results show that Vivado's router is unsurprisingly superior to RSVRoute. However, the goal of RSVRoute was not to compete with Vivado, but to provide a functional router for Maverick, which is meant only to compile RM designs. RSVRoute accomplishes this main goal and leaves many opportunities for improvement in the future.

\subsection{Maverick Results}

This section presents results on Maverick as a whole. The tests in this section were all executed on the same desktop computer as in the previous section. Furthermore, to evaluate Maverick's performance with different sizes of PR regions, these tests all targeted three different-sized PR regions of this device, given in Table 7.4.

Table 7.4: PR Regions Used in Tests

\begin{tabular}{|c|r|r|r|r|}
\hline Region & Tiles & Slices & LUTs & FFs \\
\hline Region A & 200 & 200 & 1600 & 1600 \\
Region B & 400 & 400 & 3200 & 3200 \\
Region C & 600 & 600 & 4800 & 4800 \\
\hline xc7z020clg400-1 & 29359 & 13300 & 106400 & 106400 \\
\hline
\end{tabular}

Each region follows the requirements for PR regions explained in Chapter 2, meaning each region contains 50 rows of tiles (the height of a clock region). The regions differ in their widths; region $\mathrm{A}$ is 4 tiles wide, region $\mathrm{B}$ is 8 tiles wide, and region $\mathrm{C}$ is 12 tiles wide. Each region is also made up of only CLB and INT tiles. Note that each region is contained within the area supported by our version of Project X-Ray, described in Section 5.4. Therefore, region $\mathrm{C}$ is the largest usable PR region on the Zynq device that Maverick can currently compile partial bitstreams for. However, many PR applications do not require PR regions much larger than this. 
As no standard set of PR benchmarks is known to the author, the PR designs used in these tests are either altered designs obtained from OpenCores [45] and Yosys [46], or newly created designs. Table 7.5 shows each PR design used, giving the name of the RM designs and their associated static designs. The static designs in these benchmarks contain little design logic of their own and are mainly used to connect I/O signals to the PR region. To test several designs without creating a more complex interface between the static region and PR region, most of the RM designs have a unique static design. However, the pynq_demo static design was used with 5 different RM designs; these designs were also used in the experimental demonstration described in Section 7.3.

Table 7.5: PR Benchmark Designs

\begin{tabular}{|l|l|}
\hline \multicolumn{1}{|c|}{ RM Design } & Static Design \\
\hline regfile & pynq_demo \\
sddac & sddac \\
transmitter & pynq_demo \\
stopwatch & pynq_demo \\
dspmac_8_24 & dspmac_8_24 \\
pattern_game & pynq_demo \\
crc32 & crc32 \\
timer_game & pynq_demo \\
dspmac_12_32 & dspmac_12_32 \\
dsp_mac_16_40 & dsp_mac_16_40 \\
cordic_10_16 & cordic_10_16 \\
\hline
\end{tabular}

\subsubsection{Static Design Phase Results}

Maverick's static design phase, introduced in Chapter 5, consists of (1) the creation of a static design in Vivado, (2) the creation of a partial device for the targeted PR region in RapidSmith2, and (3) the creation of a Project X-Ray bitstream database. Detailed run-time results are not presented for the second step, the creation of a partial device, as the more time consuming process is the required creation of an initial RapidSmith2 device model for the full device, discussed in [4]. Once this full device model exists, creating a partial device model can be done very quickly. For regions A, B, and C, the partial device model is created in under 35 seconds. Additionally, this section does not give results on the third step in which a Project X-Ray bitstream database is 
created. However, it should be noted that creating a complete database takes several hours. This step should be performed for the largest region of the device as possible so it does not need to be unnecessarily repeated.

During the first step of this phase, vital static design information about partition pins, routes to and from partition pins, used site route-throughs, and used PIPs must be exported from Vivado. This static design information is exported using new Tcl procedures added to the Vivado Design Interface (VDI) in Tincr, described in Chapter 4.

Table 7.6: Static Design Resource Usage

\begin{tabular}{|c|c|c|c|c|}
\hline $\begin{array}{c}\text { Static } \\
\text { Design }\end{array}$ & Region & $\begin{array}{l}\text { Part. } \\
\text { Pins }\end{array}$ & $\begin{array}{c}\text { Route- } \\
\text { throughs }\end{array}$ & PIPs \\
\hline \multirow{3}{*}{ sddac } & $\mathrm{A}$ & \multirow{3}{*}{19} & 14 & 121 \\
\hline & B & & 0 & 0 \\
\hline & $\mathrm{C}$ & & 1 & 90 \\
\hline \multirow{3}{*}{ pynq_demo } & $\bar{A}$ & \multirow{3}{*}{37} & 0 & 0 \\
\hline & B & & 0 & 0 \\
\hline & $\mathrm{C}$ & & 0 & 0 \\
\hline \multirow{3}{*}{$\operatorname{crc} 32$} & A & \multirow{3}{*}{42} & 0 & 0 \\
\hline & B & & 0 & 0 \\
\hline & $\mathrm{C}$ & & 0 & 137 \\
\hline \multirow{3}{*}{ dsp_mac_8_24 } & $\overline{\mathrm{A}}$ & \multirow{3}{*}{44} & 0 & 0 \\
\hline & B & & 0 & 0 \\
\hline & $\mathrm{C}$ & & 0 & 0 \\
\hline \multirow{3}{*}{ cordic $\_10 \_16$} & A & \multirow{3}{*}{50} & 0 & 0 \\
\hline & B & & 0 & 0 \\
\hline & $\mathrm{C}$ & & 0 & 0 \\
\hline \multirow{3}{*}{ dsp_mac_12_32 } & A & \multirow{3}{*}{60} & 0 & 0 \\
\hline & B & & 2 & 84 \\
\hline & $\mathrm{C}$ & & 0 & 0 \\
\hline \multirow{3}{*}{ dsp_mac_16_40 } & $\mathrm{A}$ & \multirow{3}{*}{76} & 0 & 0 \\
\hline & B & & 0 & 0 \\
\hline & $\mathrm{C}$ & & 0 & 0 \\
\hline
\end{tabular}

Because Tcl can be prohibitively slow [36], run-times for exporting the static design information from the static designs in Table 7.4 were measured and are presented in this subsection. As the static designs used in this subsection do not contain much design logic, some of them were manually modified to use PIPs and site route-throughs in the PR region to make the design set more representative of real PR designs. 
Table 7.7: VDI Static Design Export Times (in Seconds)

\begin{tabular}{|c|c|c|c|c|c|c|}
\hline $\begin{array}{c}\text { Static } \\
\text { Design }\end{array}$ & Region & $\begin{array}{l}\text { Part. } \\
\text { Pins }\end{array}$ & $\begin{array}{l}\text { Static } \\
\text { Routes }\end{array}$ & $\begin{array}{l}\text { Route- } \\
\text { throughs }\end{array}$ & PIPs & Total \\
\hline \multirow{3}{*}{ sddac } & $\overline{\mathrm{A}}$ & 0.03 & 0.03 & 0.92 & 2.27 & 3.25 \\
\hline & B & 0.03 & 0.03 & 0.51 & 0.45 & 1.01 \\
\hline & $\mathrm{C}$ & 0.03 & 0.03 & 0.65 & 1.30 & 2.01 \\
\hline \multirow{3}{*}{ pynq_demo } & A & 0.05 & 0.09 & 0.35 & 0.27 & 0.70 \\
\hline & B & 0.08 & 0.09 & 0.61 & 0.50 & 1.27 \\
\hline & $\mathrm{C}$ & 0.09 & 0.09 & 0.88 & 0.71 & 1.68 \\
\hline \multirow{3}{*}{$\operatorname{crc} 32$} & $\bar{A}$ & 0.04 & 0.06 & 0.30 & 0.22 & 0.57 \\
\hline & B & 0.04 & 0.06 & 0.59 & 0.44 & 1.12 \\
\hline & $\mathrm{C}$ & 0.06 & 0.09 & 0.92 & 3.26 & 4.33 \\
\hline \multirow{3}{*}{ dsp_mac_8_24 } & A & 0.06 & 0.06 & 0.60 & 0.11 & 0.49 \\
\hline & B & 0.04 & 0.06 & 0.40 & 0.23 & 0.74 \\
\hline & $\mathrm{C}$ & 0.04 & 0.09 & 0.84 & 0.34 & 1.32 \\
\hline \multirow{3}{*}{ cordic_10_16 } & $\bar{A}$ & 0.05 & 0.07 & 0.29 & 0.21 & 0.57 \\
\hline & B & 0.05 & 0.09 & 0.57 & 0.44 & 1.15 \\
\hline & $\mathrm{C}$ & 0.05 & 0.07 & 0.87 & 0.66 & 1.65 \\
\hline \multirow{3}{*}{ dsp_mac_12_32 } & A & 0.07 & 0.11 & 0.25 & 0.22 & 0.65 \\
\hline & B & 0.08 & 0.08 & 0.65 & 1.73 & 2.54 \\
\hline & $\mathrm{C}$ & 0.06 & 0.09 & 0.62 & 0.66 & 1.42 \\
\hline \multirow{3}{*}{ dsp_mac_16_40 } & $\mathrm{A}$ & 0.08 & 0.11 & 0.31 & 0.22 & 0.72 \\
\hline & B & 0.07 & 0.15 & 0.57 & 0.44 & 1.23 \\
\hline & $\mathrm{C}$ & 0.08 & 0.12 & 0.88 & 0.66 & 1.72 \\
\hline
\end{tabular}

Table 7.6 gives statistics about the resources used in each static design implemented in regions $\mathrm{A}, \mathrm{B}$, and $\mathrm{C}$. Table 7.7 gives the run-times for exporting them; the run-times were measured once for each static design, but little to no variation in run-time can be observed between runs for the same design. These results show that exporting the static design information is generally quite fast, completing in under 5 seconds for the tested designs and PR regions. As one would expect, the total run-time increases with the size of the PR region, and to a lesser extent, the number of partition pins in the design. Also of note is the fact that the time to find site route-throughs and PIPs used by the static design dominates the total run-time, even if no route-throughs or PIPs are being used by the static design. The time to find the PIPs increased further, by about $3 x-6 x$, when PIPs were actually used by the static design. The increased amount of time needed to obtain these pieces of data is due to the fact that Vivado's Tcl interface does not provide easy access to this information, requiring the more time-consuming methods explained in Subsection 4.2.2. 


\subsubsection{Reconfigurable Module (RM) Phase Results}

This subsection presents detailed results of compiling the RM designs in Table 7.5 with Maverick on a desktop computer. These RM designs were all compiled a single time using the corresponding generated static design information described in the previous subsection.

Table 7.8: Quality of the Implemented RM Benchmarks

\begin{tabular}{|c|c|c|c|c|c|}
\hline RM Design & \# Cells & \# Nets & Region & Slice Util. & Wire-length \\
\hline \multirow{3}{*}{ regfile } & \multirow{3}{*}{77} & \multirow{3}{*}{49} & $\mathrm{~A}$ & $14.00 \%$ & 864 \\
\hline & & & B & $7.00 \%$ & 1445 \\
\hline & & & $\mathrm{C}$ & $4.67 \%$ & 1121 \\
\hline \multirow{3}{*}{ sddac } & \multirow{3}{*}{276} & \multirow{3}{*}{343} & A & $24.50 \%$ & 1906 \\
\hline & & & B & $12.25 \%$ & 1674 \\
\hline & & & $\mathrm{C}$ & $8.17 \%$ & 1667 \\
\hline \multirow{3}{*}{ transmitter } & \multirow{3}{*}{269} & \multirow{3}{*}{251} & A & $27.00 \%$ & 2224 \\
\hline & & & B & $13.50 \%$ & 2187 \\
\hline & & & $\mathrm{C}$ & $9.00 \%$ & 2595 \\
\hline \multirow{3}{*}{ stopwatch } & \multirow{3}{*}{340} & \multirow{3}{*}{340} & A & $28.00 \%$ & 3109 \\
\hline & & & B & $14.00 \%$ & 3167 \\
\hline & & & $\mathrm{C}$ & $9.33 \%$ & 3205 \\
\hline \multirow{3}{*}{ dsp_mac_8_24 } & \multirow{3}{*}{401} & \multirow{3}{*}{411} & A & $30.00 \%$ & 7408 \\
\hline & & & B & $15.00 \%$ & 6057 \\
\hline & & & $\mathrm{C}$ & $10.00 \%$ & 6856 \\
\hline \multirow{3}{*}{ pattern_game } & \multirow{3}{*}{473} & \multirow{3}{*}{497} & A & $39.00 \%$ & 4202 \\
\hline & & & B & $19.50 \%$ & 4298 \\
\hline & & & $\mathrm{C}$ & $13.00 \%$ & 4164 \\
\hline \multirow{3}{*}{$\operatorname{crc} 32$} & \multirow{3}{*}{179} & \multirow{3}{*}{403} & A & -1 & $-^{2}$ \\
\hline & & & B & $19.75 \%$ & 3558 \\
\hline & & & $\mathrm{C}$ & $13.17 \%$ & 4381 \\
\hline \multirow{3}{*}{ timer_game } & \multirow{3}{*}{684} & \multirow{3}{*}{673} & $\mathrm{~A}$ & $52.00 \%$ & 8462 \\
\hline & & & B & $26.00 \%$ & 7468 \\
\hline & & & $\mathrm{C}$ & $17.33 \%$ & 7397 \\
\hline \multirow{3}{*}{ dsp_mac_12_32 } & \multirow{3}{*}{693} & \multirow{3}{*}{711} & $\mathrm{~A}$ & $54.00 \%$ & - \\
\hline & & & B & $27.00 \%$ & 14029 \\
\hline & & & $\mathrm{C}$ & $18.00 \%$ & 13761 \\
\hline \multirow{3}{*}{ dsp_mac_16_40 } & \multirow{3}{*}{1067} & & $\mathrm{~A}$ & $89.50 \%$ & - \\
\hline & & 1092 & B & $45.25 \%$ & 33500 \\
\hline & & & $\mathrm{C}$ & $30.17 \%$ & 27387 \\
\hline & & & $\mathrm{A}$ & - & - \\
\hline cordic $\_10 \_16$ & 2732 & 3483 & B & $84.25 \%$ & - \\
\hline & & & $\mathrm{C}$ & $56.17 \%$ & 42351 \\
\hline
\end{tabular}

${ }^{1} \mathrm{~A}$ " _-" in the Slice Util. column indicates packing/placement failure.

${ }^{2} \mathrm{~A}$ " - " in the Wire-length column indicates routing failure. 
Table 7.8 gives information about the size and quality of the compiled benchmarks, implemented in regions A, B, and C. Notably, this shows that some designs failed to compile under smaller PR regions. For instance, RSVPack failed to pack the cordic_10_16 and crc32 benchmarks when region A was being used as the PR region; this occurred because RSVPack required more sites to pack the design than existed in the region. Other designs failed to route in under 35 minutes on a desktop computer and were therefore considered to have failed. This occurred when the PR region was small or when slice utilization was high. These results show the importance of choosing a PR region that can properly accommodate the circuits that will be implemented within it.

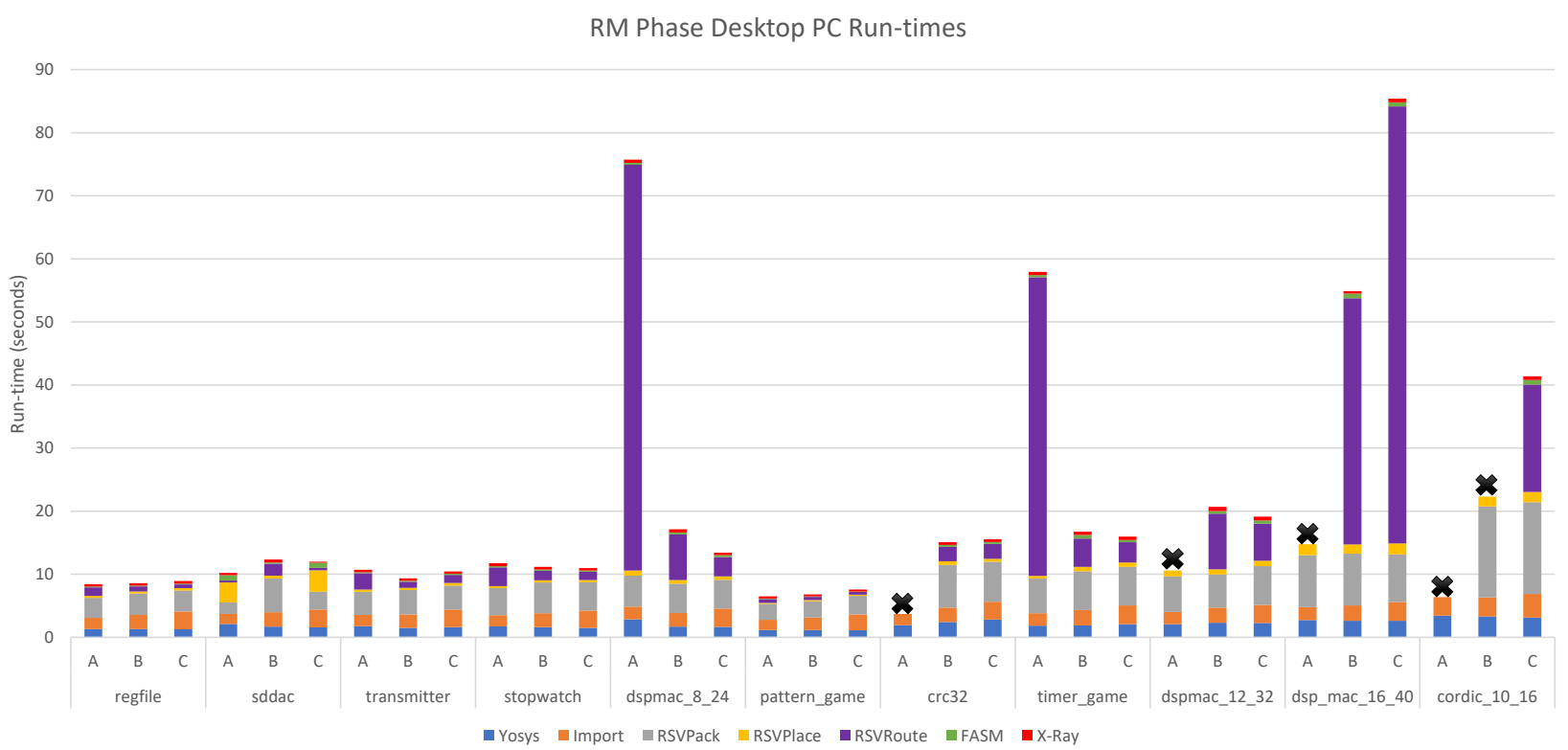

Figure 7.1: RM Phase Run-Times on a Desktop PC (in Seconds)

One would expect the wire-length to only decrease as the size of the target PR region grew. However, no clear trends on the resulting wire-length can be seen from Table 7.8. The total wire-length is affected not only by the router, but by every step of the CAD flow. To determine the cause of these results, testing was performed to verify that both RSVPack and RSVRoute are deterministic. Additionally, RSVPack was observed to produce the same packing results for a design as the PR region size increased. However, RSVPlace, a simple simulated annealing placer, produced non-deterministic results at the time of this work. Because of this, RSVPlace sometimes produced better placements for smaller PR regions than larger ones. Therefore, the main cause 
behind the lack of a trend in the wire-length results is RSVPlace. If RSVPlace is improved and made deterministic, it is expected that the wire-length results would improve as PR regions grow.

Table 7.9: RM Phase Run-times on a Desktop PC (in Seconds)

\begin{tabular}{|c|c|c|c|c|c|c|c|c|c|}
\hline RM Design & Region & Yosys & Import & $\begin{array}{l}\text { RSV } \\
\text { Pack }\end{array}$ & $\begin{array}{l}\text { RSV } \\
\text { Place }\end{array}$ & $\begin{array}{l}\text { RSV } \\
\text { Route }\end{array}$ & FASM & X-Ray & Total \\
\hline \multirow{3}{*}{ regfile } & A & 1.32 & 1.78 & 3.15 & 0.33 & 1.35 & 0.13 & 0.36 & 8.41 \\
\hline & B & 1.32 & 2.21 & 3.44 & 0.30 & 0.82 & 0.12 & 0.38 & 8.59 \\
\hline & $\mathrm{C}$ & 1.28 & 2.79 & 3.34 & 0.39 & 0.57 & 0.12 & 0.43 & 8.93 \\
\hline \multirow{3}{*}{ sddac } & A & 2.10 & 1.86 & 1.82 & 3.16 & 0.32 & 0.84 & 0.37 & 10.48 \\
\hline & B & 1.69 & 2.29 & 5.32 & 0.47 & 1.90 & 0.22 & 0.43 & 12.31 \\
\hline & $\mathrm{C}$ & 1.70 & 2.83 & 2.82 & 3.39 & 0.38 & 0.85 & 0.15 & 12.11 \\
\hline \multirow{3}{*}{ transmitter } & A & 1.77 & 1.79 & 3.65 & 0.35 & 2.59 & 0.17 & 0.36 & 10.69 \\
\hline & B & 1.51 & 2.12 & 3.86 & 0.33 & 0.96 & 0.17 & 0.38 & 9.33 \\
\hline & $\mathrm{C}$ & 1.61 & 2.79 & 3.78 & 0.42 & 1.31 & 0.16 & 0.37 & 10.44 \\
\hline \multirow{3}{*}{ stopwatch } & $\mathrm{A}$ & 1.72 & 1.78 & 4.28 & 0.31 & 2.98 & 0.20 & 0.48 & 11.76 \\
\hline & B & 1.63 & 2.19 & 4.86 & 0.36 & 1.51 & 0.20 & 0.40 & 11.15 \\
\hline & $\mathrm{C}$ & 1.49 & 2.75 & 4.49 & 0.35 & 1.31 & 0.19 & 0.39 & 10.97 \\
\hline \multirow{3}{*}{ dspmac $\_8 \_24$} & $\mathrm{~A}$ & 2.84 & 2.04 & 4.89 & 0.82 & 64.33 & 0.30 & 0.52 & 75.74 \\
\hline & B & 1.69 & 2.18 & 4.64 & 0.55 & 7.24 & 0.31 & 0.50 & 15.11 \\
\hline & $\mathrm{C}$ & 1.65 & 2.89 & 4.56 & 0.54 & 3.06 & 0.30 & 0.41 & 13.41 \\
\hline \multirow{3}{*}{ pattern_game } & A & 1.14 & 1.63 & 2.54 & 0.15 & 0.61 & 0.08 & 0.33 & 6.48 \\
\hline & B & 1.16 & 2.02 & 2.52 & 0.21 & 0.51 & 0.09 & 0.31 & 6.81 \\
\hline & $\mathrm{C}$ & 1.10 & 2.53 & 2.93 & 0.18 & 0.43 & 0.08 & 0.30 & 7.55 \\
\hline \multirow{3}{*}{$\operatorname{crc} 32$} & $\mathrm{~A}$ & 1.92 & 1.80 & -1 & - & - & - & - & - \\
\hline & B & 2.41 & 2.29 & 6.78 & 0.57 & 2.29 & 0.30 & 0.43 & 15.07 \\
\hline & $\mathrm{C}$ & 1.99 & 2.77 & 6.32 & 0.48 & 2.35 & 0.33 & 0.42 & 14.65 \\
\hline \multirow{3}{*}{ timer_game } & A & 1.85 & 1.97 & 5.49 & 0.44 & 47.32 & 0.35 & 0.53 & 57.95 \\
\hline & B & 1.88 & 2.42 & 6.17 & 0.69 & 4.51 & 0.56 & 0.51 & 16.74 \\
\hline & $\mathrm{C}$ & 2.07 & 2.99 & 6.10 & 0.71 & 3.22 & 0.34 & 0.52 & 15.95 \\
\hline \multirow{3}{*}{ dspmac_12_32 } & $\mathrm{A}$ & 2.08 & 1.92 & 5.65 & 0.95 & - & - & - & - \\
\hline & B & 2.13 & 2.36 & 5.28 & 0.83 & 8.78 & 0.45 & 0.64 & 20.47 \\
\hline & $\mathrm{C}$ & 2.25 & 2.87 & 6.15 & 0.86 & 5.89 & 0.48 & 0.62 & 19.12 \\
\hline \multirow{3}{*}{ dsp_mac_16_40 } & A & 2.73 & 2.05 & 8.22 & 1.77 & - & - & - & - \\
\hline & B & 2.62 & 2.43 & 8.22 & 1.46 & 39.03 & 0.73 & 0.39 & 54.88 \\
\hline & $\mathrm{C}$ & 2.61 & 3.00 & 7.52 & 1.74 & 69.29 & 0.68 & 0.57 & 85.41 \\
\hline \multirow{3}{*}{ cordic_10_16 } & A & 3.42 & 2.93 & - & - & - & - & - & - \\
\hline & B & 3.30 & 3.01 & 14.43 & 1.57 & - & - & - & - \\
\hline & $\mathrm{C}$ & 3.11 & 3.75 & 14.54 & 1.63 & 17.03 & 0.72 & 0.59 & 41.37 \\
\hline
\end{tabular}

${ }^{1} \mathrm{~A}$ " - " indicates that the step failed to complete.

Figure 7.1 gives the run-times required to compile these benchmarks on a desktop computer, with Table 7.9 giving the exact times. These results show that in most cases, RSVRoute 
dominates the run-time, taking as much as $85 \%$ of the total run-time in the worst case. However, the percentage of the run-time used by RSVRoute was also as low as 3\%. These results show that RSVRoute's run-time was generally much more competitive for the smaller benchmarks and for larger PR regions in which more choices for routing were available. These results support the findings on RSVRoute described in Section 7.1.

Following RSVRoute, RSVPack is the second largest bottleneck in the RM phase; it accounts for more run-time than RSVRoute in the smallest benchmarks. The time required to import designs, and their respective PR regions, into RapidSmith2 is also noticeable. On average, designs using region $\mathrm{B}$ (which is twice the size as region $\mathrm{A}$ ) took $1.21 \mathrm{x}$ longer to import than designs using region $\mathrm{A}$, and designs using region $\mathrm{C}$ (which is triple the size of region $\mathrm{A}$ ) took $1.52 \mathrm{x}$ longer than designs using region $\mathrm{A}$.

The results presented in this section show that additional work can be done to make Maverick's RM phase more efficient. However, these results still show that Maverick was able to complete all phases of the FPGA CAD flow when PR regions of sufficient size were used. These results serve as initial proof that RM designs for Xilinx devices can be compiled from Verilog to bitstream with the use of only third-party tools.

\subsection{Maverick on the PYNQ-Z1}

As stated in Chapter 1, one of the goals of this work was to enable the compilation of partial bitstreams on an embedded device. Many of the design decisions that went into creating Maverick were made to this end. Digilent's PYNQ-Z1 board was chosen to demonstrate the ability of Maverick's RM phase to run on embedded systems. The PYNQ-Z1 is designed specifically as a hardware platform for Xilinx's open-source PYNQ system. This board contains the xc7z020clg400-1 Zynq SoC, which tightly couples an ARM processing system (PS) and programmable logic (PL). The ARM CPU is an ARM Cortex-A9, which runs at $650 \mathrm{MHz}$, and the board also contains $512 \mathrm{MB}$ of RAM.

The PYNQ system, including Ubuntu Linux, the open-source Jupyter notebook infrastruc-

ture, and a web server, runs on the ARM CPU. This allows the PYNQ system to be able to be easily assessed from any computing platform and operating system via a web interface. The PYNQ system running on Ubuntu requires roughly $120 \mathrm{MB}$ of RAM, leaving about $392 \mathrm{MB}$ for Maverick. 
Subsection 7.3.1 first presents the results of executing Maverick's RM phase on Digilent's PYNQ-Z1 board. These results are analyzed and compared to the results from Subsection 7.2.2. Subsection 7.3.2 then describes an experimental demo in which Maverick's RM phase was integrated more into the PYNQ system. The designs created for this demo were programmed to the PYNQ-Z1's FPGA and were observed to function correctly.

\subsubsection{RM Phase Results}

The same RM benchmarks used in Subsection 7.2.2 were also compiled once each on the PYNQ-Z1's Ubuntu installation over an SSH connection. The same static design data, presented in Subsection 7.2.1, were used for these RM designs. As expected, the quality of the results remains unchanged from the results obtained on a desktop computer, seen in Table 7.8. However, the run-times for compiling the designs on the PYNQ-Z1, shown in Figure 7.2 and Table 7.10, differ significantly.

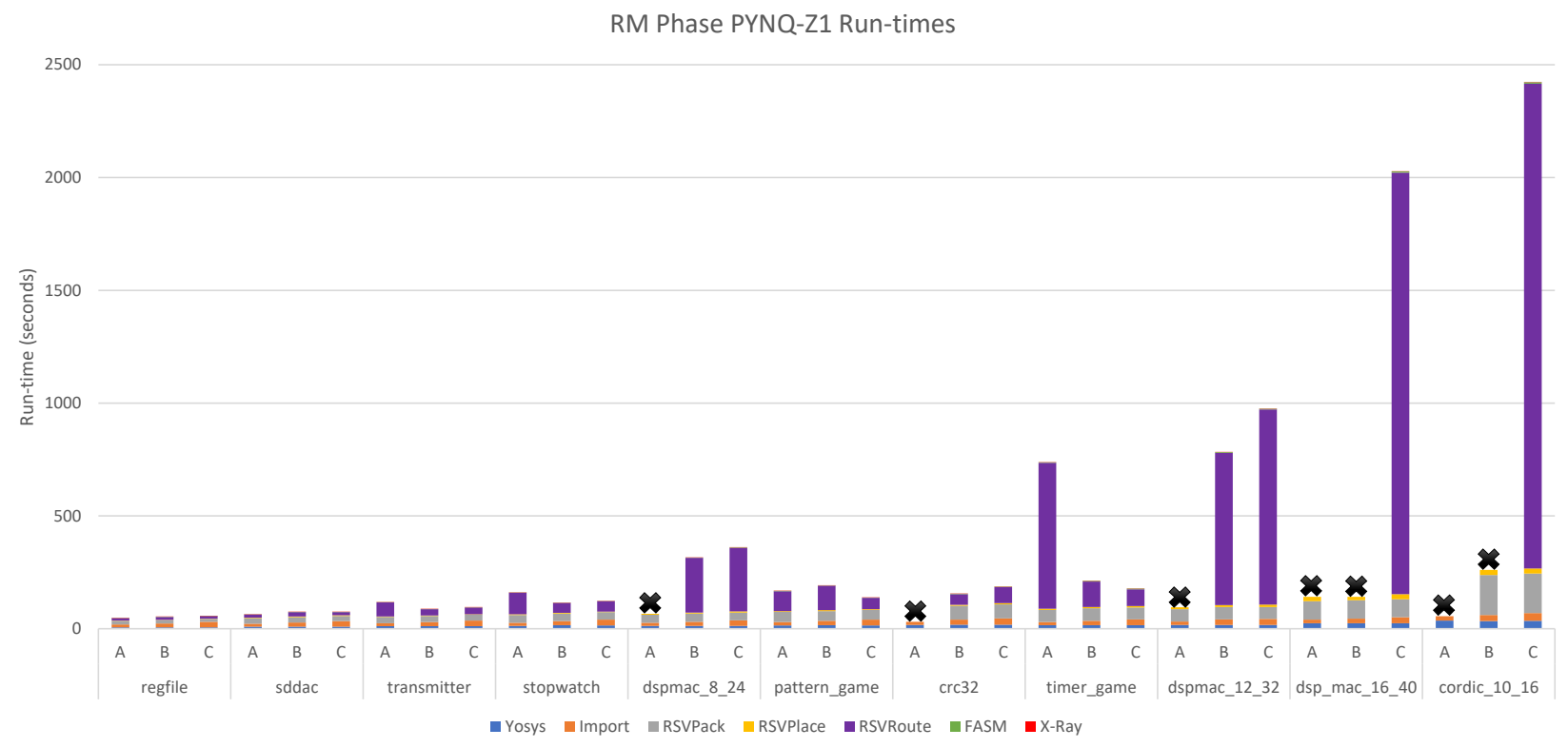

Figure 7.2: RM Phase Run-Times on the PYNQ-Z1 (in Seconds)

The mean increase in total run-time was $17.6 \mathrm{x}$, while the maximum increase was by $58.54 \mathrm{x}$ for the compilation of the cordic_10_16 benchmark targeting region $\mathrm{C}$. The mean increases for Yosys, RapidSmith2 import, RSVPack, RSVPlace, RSVRoute, FASM generation, and bitstream 
Table 7.10: RM Phase Run-times on the PYNQ-Z1 (in Seconds)

\begin{tabular}{|c|c|c|c|c|c|c|c|c|c|}
\hline RM Design & Region & Yosys & Import & $\begin{array}{l}\text { RSV } \\
\text { Pack }\end{array}$ & $\begin{array}{l}\text { RSV } \\
\text { Place }\end{array}$ & $\begin{array}{l}\text { RSV } \\
\text { Route }\end{array}$ & FASM & X-Ray & Total \\
\hline \multirow{3}{*}{ regfile } & $\mathrm{A}$ & 6.46 & 12.44 & 15.89 & 0.97 & 11.13 & 0.57 & 0.15 & 47.61 \\
\hline & B & 6.46 & 17.08 & 15.20 & 1.04 & 12.97 & 0.56 & 0.18 & 53.48 \\
\hline & $\mathrm{C}$ & 6.51 & 23.99 & 13.65 & 1.12 & 11.16 & 0.55 & 0.19 & 57.17 \\
\hline \multirow{3}{*}{ sddac } & $\mathrm{A}$ & 8.81 & 13.85 & 23.87 & 2.22 & 14.57 & 0.91 & 0.16 & 64.41 \\
\hline & B & 9.23 & 18.52 & 23.73 & 2.66 & 19.90 & 0.95 & 0.18 & 75.18 \\
\hline & $\mathrm{C}$ & 8.79 & 25.37 & 21.93 & 3.19 & 14.51 & 0.89 & 0.20 & 74.88 \\
\hline \multirow{3}{*}{ transmitter } & $\mathrm{A}$ & 11.46 & 13.39 & 27.55 & 1.91 & 62.91 & 1.07 & 0.15 & 118.43 \\
\hline & $\mathrm{B}$ & 11.47 & 18.02 & 26.58 & 2.43 & 28.38 & 1.00 & 0.18 & 88.05 \\
\hline & $\mathrm{C}$ & 11.47 & 25.03 & 25.45 & 2.42 & 30.34 & 1.00 & 0.19 & 95.89 \\
\hline \multirow{3}{*}{ stopwatch } & A & 12.8 & 13.75 & 34.79 & 2.66 & 95.65 & 1.22 & 0.15 & 161.02 \\
\hline & B & 15.38 & 18.41 & 33.76 & 3.21 & 43.66 & 1.21 & 0.19 & 115.82 \\
\hline & $\mathrm{C}$ & 14.24 & 25.85 & 33.25 & 3.06 & 46.06 & 1.18 & 0.20 & 123.83 \\
\hline \multirow{3}{*}{ dspmac_8_24 } & $\mathrm{A}$ & 12.01 & 14.13 & 35.84 & 4.60 & -1 & - & - & - \\
\hline & B & 12.06 & 18.80 & 35.95 & 4.38 & 242.90 & 1.92 & 0.18 & 316.18 \\
\hline & $\mathrm{C}$ & 12.00 & 25.70 & 34.13 & 4.90 & 282.15 & 1.92 & 0.20 & 361.00 \\
\hline \multirow{3}{*}{ pattern_game } & A & 14.79 & 15.09 & 44.94 & 3.17 & 88.66 & 1.49 & 0.15 & 168.29 \\
\hline & B & 15.41 & 19.35 & 43.63 & 3.74 & 108.65 & 1.51 & 0.15 & 192.45 \\
\hline & $\mathrm{C}$ & 14.82 & 25.69 & 42.71 & 3.73 & 50.04 & 1.45 & 0.19 & 138.63 \\
\hline \multirow{3}{*}{$\operatorname{crc} 32$} & $\mathrm{~A}$ & 16.59 & 13.95 & - & - & - & - & - & - \\
\hline & B & 17.47 & 22.86 & 61.28 & 4.78 & 47.25 & 2.07 & 0.18 & 155.89 \\
\hline & $\mathrm{C}$ & 17.46 & 28.80 & 62.13 & 4.95 & 70.71 & 2.06 & 0.21 & 186.33 \\
\hline \multirow{3}{*}{ timer_game } & $\mathrm{A}$ & 15.42 & 14.57 & 53.81 & 5.33 & 646.53 & 2.48 & 0.15 & 738.28 \\
\hline & B & 15.56 & 19.30 & 56.22 & 4.84 & 113.53 & 2.43 & 0.18 & 212.06 \\
\hline & $\mathrm{C}$ & 15.47 & 26.11 & 52.28 & 6.48 & 74.94 & 2.45 & 0.19 & 177.93 \\
\hline \multirow{3}{*}{ dspmac_12_32 } & $\mathrm{A}$ & 16.74 & 14.91 & 54.58 & 9.36 & - & - & - & - \\
\hline & $\mathrm{B}$ & 17.33 & 24.23 & 55.22 & 7.77 & 675.44 & 3.31 & 0.16 & 783.46 \\
\hline & $\mathrm{C}$ & 16.74 & 26.41 & 53.15 & 10.99 & 865.02 & 3.35 & 0.19 & 975.85 \\
\hline \multirow{3}{*}{ dsp_mac $\_16 \_40$} & $\mathrm{~A}$ & 24.54 & 15.65 & 81.61 & 19.99 & - & - & - & - \\
\hline & B & 24.74 & 20.28 & 80.87 & 15.99 & - & - & - & - \\
\hline & $\mathrm{C}$ & 24.47 & 27.05 & 79.45 & 22.08 & 1868.76 & 5.66 & 0.20 & 2027.67 \\
\hline \multirow{3}{*}{ cordic_10_16 } & $\mathrm{A}$ & 36.04 & 19.70 & - & - & - & - & - & - \\
\hline & B & 34.19 & 26.20 & 177.31 & 23.46 & - & - & - & - \\
\hline & $\mathrm{C}$ & 35.00 & 34.77 & 174.81 & 22.13 & 2149.07 & 6.40 & 0.21 & 2422.39 \\
\hline
\end{tabular}

${ }^{1} \mathrm{~A}$ " - " indicates that the step failed to complete.

generation with Project X-Ray, were 8.17x, 11.27x, 8.72x, 9.61x, 51.12x, 7.36x, and 0.42x, respectively. As evidenced by its 51.12x increase in run-time, RSVRoute clearly suffers the most when executed on a resource-constrained platform like the PYNQ-Z1. However, the increased run-time for all other components is also significant. 
Table 7.11: RM Phase Peak Memory Usage on the PYNQ-Z1 (in MB)

\begin{tabular}{|c|c|c|c|c|}
\hline RM Design & Region & Yosys & RS2 & X-Ray \\
\hline \multirow{3}{*}{ regfile } & A & 20.02 & 184.09 & 3.45 \\
\hline & B & 20.03 & 190.57 & 3.57 \\
\hline & $\mathrm{C}$ & 20.08 & 267.69 & 3.72 \\
\hline \multirow{3}{*}{ sddac } & A & 21.14 & 181.62 & 3.50 \\
\hline & B & 21.14 & 191.65 & 3.62 \\
\hline & $\mathrm{C}$ & 21.17 & 272.11 & 3.81 \\
\hline \multirow{3}{*}{ transmitter } & $\bar{A}$ & 21.49 & 195.33 & 3.44 \\
\hline & B & 21.54 & 193.48 & 3.45 \\
\hline & $\mathrm{C}$ & 21.64 & 272.26 & 3.60 \\
\hline \multirow{3}{*}{ stopwatch } & A & 21.69 & 190.35 & 3.43 \\
\hline & B & 21.63 & 194.50 & 3.56 \\
\hline & $\mathrm{C}$ & 21.69 & 275.68 & 3.64 \\
\hline \multirow{3}{*}{ dspmac_8_24 } & A & 26.75 & 204.48 & 3.51 \\
\hline & B & 26.65 & 204.35 & 3.68 \\
\hline & $\mathrm{C}$ & 26.64 & 294.22 & 3.80 \\
\hline \multirow{3}{*}{ pattern_game } & A & 21.52 & 188.27 & 3.45 \\
\hline & B & 21.69 & 200.89 & 3.49 \\
\hline & $\mathrm{C}$ & 21.53 & 278.25 & 3.65 \\
\hline \multirow{3}{*}{$\operatorname{crc} 32$} & $\mathrm{~A}$ & 21.26 & -1 & - \\
\hline & B & 21.10 & 213.27 & 3.61 \\
\hline & $\mathrm{C}$ & 21.20 & 295.05 & 3.87 \\
\hline \multirow{3}{*}{ timer_game } & A & 23.12 & 178.00 & 3.49 \\
\hline & B & 23.15 & 228.38 & 3.58 \\
\hline & $\mathrm{C}$ & 23.11 & 276.55 & 3.67 \\
\hline \multirow{3}{*}{ dspmac_12_32 } & $\bar{A}$ & 31.07 & - & - \\
\hline & B & 31.08 & 一 & - \\
\hline & $\mathrm{C}$ & 31.03 & 297.37 & 3.78 \\
\hline \multirow{3}{*}{ dsp_mac $\_16 \_40$} & A & 37.40 & - & - \\
\hline & B & 37.39 & - & - \\
\hline & $\mathrm{C}$ & 37.43 & 309.23 & 3.82 \\
\hline \multirow{3}{*}{ cordic_10_16 } & $\bar{A}$ & 36.17 & - & - \\
\hline & B & 36.17 & 260.57 & - \\
\hline & C & 36.13 & 348.87 & 3.81 \\
\hline
\end{tabular}

${ }^{1} \mathrm{~A}$ "—." indicates that the step failed to complete.

This increased run-time is unsurprising, considering the differences in the processors and the amount of available RAM. In order to gain further insight on the limiting factors of executing Maverick in resource-constrained environments such as the PYNQ-Z1, the peak memory usage for each major step of the Maverick flow was also measured with the GNU time command. Table 7.11 
gives these values in MB for each major step of Maverick. The peak memory for each design was measured once, but the results are fairly consistent between runs for the same design.

As one would expect, RapidSmith2 and Project X-Ray require more memory when larger PR regions are used, while Yosys is unaffected. These results also show that the RapidSmith2based parts of Maverick, which execute together as one program, require significantly more memory than Yosys and Project X-Ray. The higher memory requirements for RapidSmith2 are mainly caused by the memory needed for the detailed device representation as well as the many data structures shared by RSVPack, RSVPlace, and RSVRoute.

Although the RM phase's performance suffers on the PYNQ-Z1, the very fact that it was able to run at all is significant. Furthermore, these results answer the main questions posed at the beginning of this thesis-proving that a non-vendor PR CAD flow for Xilinx devices is possible and that such a flow can run on embedded devices. Uniquely, these results also prove that an SoC can actually generate bitstreams that can later be used to reconfigure itself.

\subsubsection{Experimental Demo}

An experimental demonstration for the PYNQ-Z1 board was created to further evaluate Maverick's correctness and the possibilities it enables. This subsection gives the results of this demonstration, in which Maverick was integrated with Xilinx's open-source PYNQ system. As previously discussed, the Zynq SoC on the PYNQ-Z1 combines an ARM processing system (PS) and programmable logic (PL). The PYNQ system runs on the ARM CPU and can be accessed via a web interface.

To integrate Maverick with PYNQ, we created a a simple Jupyter notebook, shown in Fig-

ure 7.3. We also replaced the PYNQ-Z1's default Zynq boot image so the FPGA is immediately programmed with an initial bitstream containing the pynq_demo static design. This allows notebook users to create simple Verilog RM designs, compile them to partial bitstreams, and then partially reconfigure the resulting bitstreams all to the same device the tools are running on. We integrated a publicly available API to the PYNQ system to allow partial reconfiguration of the Zynq's PL to be performed through the notebook [48].

Specifically, the five RM designs that use the pynq_demo static design from the previous sections, pattern_game, regfile, stopwatch, timer_game, and transmitter, were created for use in this 


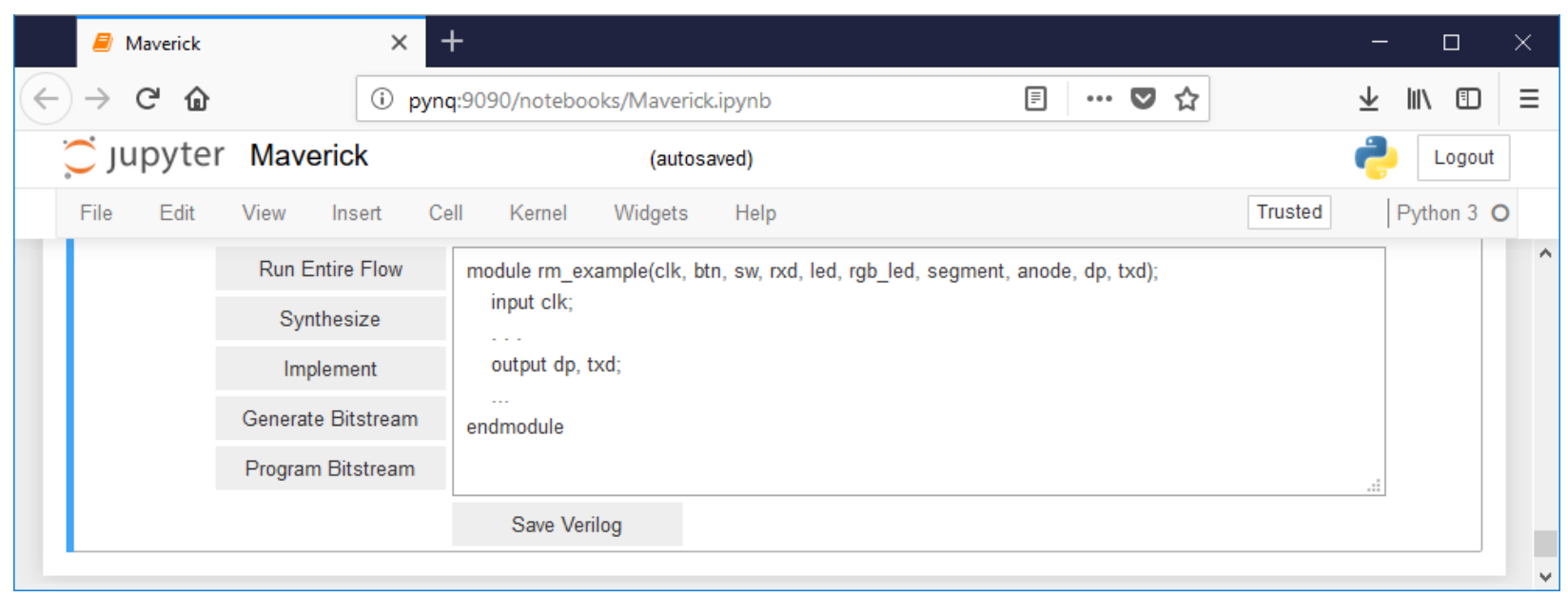

Figure 7.3: Jupyter Notebook for Maverick's RM Phase

demo. These designs represent the type of designs that might be created as part of an introductory digital systems course. Furthermore the designs also all use readily available physical I/O that can be used to verify proper operation, including buttons, switches, and LEDs. Significantly, each of these designs was compiled with Maverick and then tested and verified to work as expected on the PYNQ-Z1 board. Figure 7.4 shows the PYNQ-Z1 board after it was partially reconfigured with a partial bitstream for the stopwatch RM. These designs serve as visibly verifiable evidence of Maverick's ability to create operational partial bitstreams from Verilog.

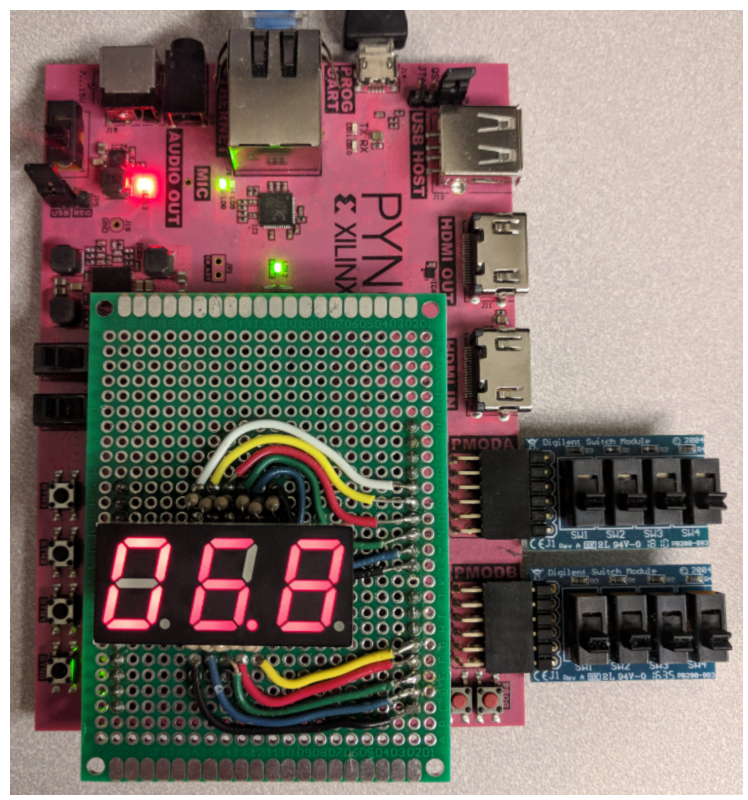

Figure 7.4: The PYNQ-Z1 Board Configured with a Stopwatch Partial Bitstream 
This experiment has continued to be built upon by researchers at BYU, namely Reilly McKendrick. Maverick can now also be used with JupyterLab, an evolution of the Jupyter Notebook. Additionally, simulation tools, including Verilator [49], have been added as part of an ongoing goal to create a stand-alone, interactive learning environment that mixes instructional materials, sample designs, HDL code, and CAD tools. 


\section{CHAPTER 8. CONCLUSION}

This work introduced Maverick, an open-source flow for compiling Xilinx 7-Series reconfigurable modules (RMs) from Verilog to partial bitstreams. This chapter concludes this thesis by summarizing the contributions of this work and by suggesting directions for potential future work.

Chapters 1, 2, and 3 of this thesis motivated this work and gave necessary background information. Potential use-cases of partial reconfiguration (PR) that are not possible with the current vendor tools were advocated for. These use cases include stand-alone educational systems as well as adaptive self-reconfiguring systems. The open-source tools that Maverick builds upon-Yosys, RapidSmith2, and Project X-Ray-were also introduced.

Chapter 4 described the additions made to RapidSmith2 and the Vivado Design Interface (VDI) to support PR, and by extension, Maverick. These additions included adding partition pin support to RapidSmith2 as well as the ability to exchange RM designs between Vivado and RapidSmith2. Importantly, all necessary static design information for RM design manipulation is included in RM RapidSmith2 checkpoints (RSCPs).

Chapter 5 gave an overview of Maverick, the main contribution of this work, and described its static design phase. Maverick is unique in that once an initial static design is made, it can operate independently of vendor tools. Next, Chapter 6 described the RM phase of Maverick, describing each individual step in depth. A new RapidSmith2-based router, RSVRoute, which was created to support Maverick, was also introduced and described in detail. Additions to RapidSmith2, RSVPack, RSVPlace, and Project X-Ray were also discussed. These additions included xc7PartialPatch, a program built upon Project X-Ray that can create new partial bitstreams.

Lastly, Chapter 7 presented the results of various tests and experiments that were done to evaluate this work. First, the runtimes of exporting static design information from Vivado were shared and then the performance and quality of RSVRoute was analyzed. Maverick as a whole was then tested with various benchmark designs; the runtimes and peak memory usage of 
Maverick running on a desktop computer and on the PYNQ-Z1 board were given. Finally, the results of integrating Maverick into the PYNQ system were shared. In this last experiment, we gave the results of executing Maverick on the the PYNQ-Z1's ARM processor to compile a collection of Verilog designs to partial bitstreams. These resulting bitstreams were all configured and verified in hardware on the PYNQ-Z1's FPGA fabric, demonstrating the feasibility of a single-chip system that can both compile HDL designs to bitstreams and then configure them onto its own programmable fabric.

\subsection{Future Work}

While much effort was put into Maverick, it is mainly a proof of concept. Maverick has shown the feasibility of third-party Xilinx CAD flows-specifically the feasibility of a third-party partial reconfiguration flow. In addition, Maverick has shown that such a flow can run on resourceconstrained embedded systems, enabling new use-cases for partial reconfiguration. However, several potential future opportunities to build upon Maverick exist.

Firstly, Maverick's static design phase could be enhanced. Currently, Maverick has only been used with PR designs containing a single PR region. Extending the static design phase to support multiple PR regions should be a modest change to the current system. The possibility of creating a static design without Vivado could also be further investigated.

The individual components of Maverick's RM phase can also be extended and improved in many ways. A significant extension would be support for Xilinx architectures beyond 7-Series, such as the Zynq UltraScale+. The RapidSmith2-based tools would require updates, but they would be modest considering RapidSmith2's existing support for newer Xilinx architectures. However, Yosys and especially Project X-Ray would require much more work to support newer Xilinx architectures.

Furthermore, RSVPack, RSVPlace, and RSVRoute could all be significantly improved with the addition of timing-driven features. However, Xilinx does not make its wire delay model public. Unless more detailed wire delay information becomes more readily available, wire delays would therefore likely need to be approximated by these tools.

Maverick's simulated annealing placer, RSVPlace, could also specifically be improved. Firstly, RSVPlace could be changed to be able to produce deterministic results between runs. The 
quality of the placement could likely be improved with the incorporation of analytical techniques as well. Furthermore, while basic changes were made to RSVPlace to support RM designs were made, RSVPlace's RM support could be improved to incorporate the placement of pre-existing partition pins into its placement of cells.

Improvements specific to RSVRoute could also be made. The run-time of RSVRoute could be greatly improved with the use of more efficient data structures. Additionally, RSVRoute's A* maze router could be improved with the use of more accurate wire type and length information. A more exhaustive sweep of RSVRoute's parameters could also be performed to find optimal settings.

Support for some device features was originally left out of RSVRoute and the FASM generator, due to a lack of support for these features from Yosys and Project X-Ray. However, both Yosys and Project X-Ray have continued to be actively developed. Yosys is being updated to better support Xilinx devices and can now map to a larger set of Xilinx primitives. Additionally, Project X-Ray now supports more devices and device primitives. The format of Project X-Ray's bitstream databases and FASM format has also been changed and improved. RSVRoute and the FASM generator could therefore be updated to support these continuing updates to Yosys and Project X-Ray.

Future work for Maverick also includes making use of it in different applications. For instance, it would be very interesting to build a self-adaptive system upon Maverick that could reconfigure itself in response to environment conditions. And, as already described in Chapter 7, Maverick's use in educational systems can continue to be worked on. Such an educational system could grow to include instructional materials as well as additional analysis and visualization tools. We believe an all-in-one system such as this could become a great asset in teaching digital design. 


\section{REFERENCES}

[1] C. Wolf, "Yosys Open SYnthesis Suite,” http://www.clifford.at/yosys/. ii, 2, 22, 23

[2] T. D. Haroldsen, B. E. Nelson, and B. Hutchings, "RapidSmith 2: A Framework for BEL-level CAD Exploration on Xilinx FPGAs," in Proceedings of the 2015 ACM/SIGDA International Symposium on Field-Programmable Gate Arrays, ser. FPGA '15. New York, NY, USA: ACM, 2015, pp. 66-69. [Online]. Available: http: //doi.acm.org/10.1145/2684746.2689085 ii, 1, 22, 24

[3] Project X-Ray. [Online]. Available: https://prjxray.readthedocs.io ii, 2, 22, 27, 70

[4] B. E. Nelson, T. J. Townsend, and T. D. Haroldsen, "RAPIDSMITH2 - A Library for Low-level Manipulation of Vivado Designs at the Cell/BEL Level," Brigham Young University, Tech. Rep., 2018. [Online]. Available: https://github.com/byuccl/rapidsmith2/ vii, 11, 26, 31, 36, 37, 39, 40, 78

[5] Yosys Manual. [Online]. Available: http://www.clifford.at/yosys/files/yosys_manual.pdf vii, 24

[6] J. S. Swartz, V. Betz, and J. Rose, "A Fast Routability-driven Router for FPGAs," in Proceedings of the 1998 ACM/SIGDA Sixth International Symposium on Field Programmable Gate Arrays, ser. FPGA '98. New York, NY, USA: ACM, 1998, pp. 140-149. [Online]. Available: http://doi.acm.org/10.1145/275107.275134 vii, 61, 62

[7] J. Luu, J. Goeders, M. Wainberg, A. Somerville, T. Yu, K. Nasartschuk, M. Nasr, S. Wang, T. Liu, N. Ahmed, K. B. Kent, J. Anderson, J. Rose, and V. Betz, "VTR 7.0: Next Generation Architecture and CAD System for FPGAs," ACM Trans. Reconfigurable Technol. Syst., vol. 7, no. 2, pp. 6:1-6:30, Jul. 2014. [Online]. Available: http://doi.acm.org/10.1145/2617593 1, 22

[8] C. Lavin, M. Padilla, J. Lamprecht, P. Lundrigan, B. E. Nelson, and B. Hutchings, "RapidSmith: Do-It-Yourself CAD Tools for Xilinx FPGAs," in 201121 st International Conference on Field Programmable Logic and Applications, Sept 2011, pp. 349-355. 1, 22, 24

[9] N. Steiner, A. Wood, H. Shojaei, J. Couch, P. Athanas, and M. French, "Torc: Towards an Open-source Tool Flow," in Proceedings of the 19th ACM/SIGDA International Symposium on Field Programmable Gate Arrays, ser. FPGA '11. New York, NY, USA: ACM, 2011, pp. 41-44. [Online]. Available: http://doi.acm.org/10.1145/1950413.1950425 1, 22

[10] C. Lavin and A. Kaviani, "RapidWright: Enabling Custom Crafted Implementations for FPGAs," in Field-Programmable Custom Computing Machines (FCCM), 2018 IEEE 26th Annual International Symposium on, May 2018. 1, 22 
[11] Xilinx. Vivado Design Suite User Guide: Release Notes, Installation, and Licensing - UG973 (v2018.2). 1

[12] - Memory Recommendations. [Online]. Available: https://www.xilinx.com/products/ design-tools/vivado/memory.html 1

[13] B. S. White and B. E. Nelson, "Tincr - A Custom CAD Tool Framework for Vivado," in 2014 International Conference on ReConFigurable Computing and FPGAs (ReConFig14), Dec 2014, pp. 1-6. 3, 25

[14] B. S. White, "Tincr: Integrating Custom CAD Tool Frameworks with the Xilinx Vivado Design Suite," Master's thesis, Brigham Young University (BYU), Provo, Utah, Dec 2014. 9,37

[15] Xilinx. 7 Series FPGAs Clocking Resources User Guide - UG472 (v1.14). [Online]. Available: https://www.xilinx.com/support/documentation/user_guides/ug472_7Series_Clocking. pdf 11

[16] A. Nishioka. Tell Me About the .BIT File Format. [Online]. Available: http: //www.fpga-faq.com/FAQ_Pages/0026_Tell_me_about_bit_files.htm 13

[17] Xilinx. 7 Series FPGAs Configuration User Guide - UG470 (v1.13.1). [Online]. Available: https://www.xilinx.com/support/documentation/user_guides/ug470_7Series_Config.pdf 13

[18] — - Vivado Design Suite User Guide: Programming and Debugging - UG908 (v2019.1). [Online]. Available: https://www.xilinx.com/support/documentation/sw_manuals/ xilinx2019_1/ug908-vivado-programming-debugging.pdf 13

[19] A. G. Stoddard, "Configuration Scrubbing Architectures for High-Reliability FPGA Systems," Master's thesis, Brigham Young University (BYU), Provo, Utah, December 2015. 13

[20] K. Vipin and S. A. Fahmy, "Fpga dynamic and partial reconfiguration: A survey of architectures, methods, and applications," ACM Comput. Surv., vol. 51, no. 4, pp. 72:1-72:39, Jul. 2018. [Online]. Available: http://doi.acm.org/10.1145/3193827 14, 15

[21] D. Koch, Partial Reconfiguration on FPGAs: Architectures, Tools and Applications. Springer Publishing Company, Incorporated, 2012. 14, 15

[22] Xilinx. PlanAhead User Guide - UG632 (v14.6). [Online]. Available: https://www.xilinx. com/support/documentation/sw_manuals/xilinx14_7/PlanAhead_UserGuide.pdf 15

[23] — - Vivado Design Suite User Guide: Partial Reconfiguration - UG909 (v2018.3). [Online]. Available: https://www.xilinx.com/support/documentation/sw_manuals/xilinx2018_3/ ug909-vivado-partial-reconfiguration.pdf 16, 18, 21, 38

[24] M. Happe, A. Traber, and A. Keller, "Preemptive hardware multitasking in reconos," in Applied Reconfigurable Computing, K. Sano, D. Soudris, M. Hübner, and P. C. Diniz, Eds. Cham: Springer International Publishing, 2015, pp. 79-90. 21 
[25] A. Traber, "Resource-efficient Dynamic Partial Reconfiguration on FPGAs," Semester Project Report. [Online]. Available: https://pub.tik.ee.ethz.ch/students/2014-FS/ SA-2014-17.pdf 21

[26] B. E. Nelson, "Third party cad tools for fpga design—a survey of the current landscape," in Applied Reconfigurable Computing. Cham: Springer International Publishing, 2019, pp. 353-367. 22

[27] nextpnr - A Portable FPGA Place and Route Tool. [Online]. Available: https: //github.com/YosysHQ/nextpnr 22

[28] N. J. Steiner, “Autonomous Computing Systems," Ph.D. dissertation, Virginia Tech, March 2008. 22

[29] Project IceStorm. [Online]. Available: http://www.clifford.at/icestorm/ 23

[30] Arachne-pnr. [Online]. Available: https://github.com/YosysHQ/arachne-pnr/ 23

[31] Trenz Electronic. icoBoard. [Online]. Available: http://icoboard.org/ 23

[32] SymbiFlow. [Online]. Available: https://symbiflow.github.io/ 23

[33] Project Trellis. [Online]. Available: https://github.com/SymbiFlow/prjtrellis 23

[34] M. Cannon, A. Keller, and M. Wirthlin, "Improving the Effectiveness of TMR Designs on FPGAs with SEU-Aware Incremental Placement," in 2018 IEEE 26th Annual International Symposium on Field-Programmable Custom Computing Machines (FCCM), April 2018, pp. 141-148. 24

[35] O. Söll, T. Korak, M. Muehlberghuber, and M. Hutter, "EM-Based Detection of Hardware Trojans on FPGAs," in 2014 IEEE International Symposium on Hardware-Oriented Security and Trust (HOST), May 2014, pp. 84-87. 24

[36] T. J. Townsend, "Vivado Design Interface: Enabling CAD-Tool Design for Next Generation Xilinx FPGA Devices," Master's thesis, Brigham Young University (BYU), Provo, Utah, July 2017. 25,79

[37] T. D. Haroldsen, B. E. Nelson, and B. Hutchings, "Packing a Modern Xilinx FPGA Using RapidSmith," in 2016 International Conference on ReConFigurable Computing and FPGAs (ReConFig), Nov 2016, pp. 1-6. 52, 53

[38] V. Betz and J. Rose, "VPR: A New Packing, Placement and Routing Tool for FPGA Research," in Field-Programmable Logic and Applications, W. Luk, P. Y. K. Cheung, and M. Glesner, Eds. Berlin, Heidelberg: Springer Berlin Heidelberg, 1997, pp. 213-222. 53

[39] L. McMurchie and C. Ebeling, "PathFinder: A Negotiation-Based Performance-Driven Router for FPGAs," in Third International ACM Symposium on Field-Programmable Gate Arrays, Feb 1995, pp. 111-117. 54, 57, 63

[40] P. E. Hart, N. J. Nilsson, and B. Raphael, "A Formal Basis for the Heuristic Determination of Minimum Cost Paths," IEEE Transactions on Systems Science and Cybernetics, vol. 4, no. 2 , pp. 100-107, July 1968. 54, 57 
[41] E. W. Dijkstra, "A Note on Two Problems in Connexion with Graphs," Numer. Math., vol. 1, no. 1, pp. 269-271, Dec. 1959. [Online]. Available: http://dx.doi.org/10.1007/BF01386390 57

[42] J. S. Swartz, “A High-Speed Timing-Aware Router for FPGAs,” Master's thesis, University of Toronto, 1998. 60, 63, 75

[43] C. A. R. Hoare, “Quicksort,” The Computer Journal, vol. 5, no. 1, pp. 10-16, 011962. [Online]. Available: https://doi.org/10.1093/comjnl/5.1.10 62

[44] T. D. Haroldsen, "Academic Packing for Commercial FPGA Architectures", Ph.D. dissertation, Brigham Young University (BYU), Provo, Utah, July 2017. 69

[45] OpenCores. [Online]. Available: https://opencores.org/ 73, 78

[46] Yosys-bench. [Online]. Available: https://github.com/YosysHQ/yosys-bench/ 73, 78

[47] Xilinx. Vivado Design Suite User Guide: Implementation - UG904 (v2019.1). [Online]. Available: https://www.xilinx.com/content/dam/xilinx/support/documentation/sw_manuals/ xilinx2019_1/ug904-vivado-implementation.pdf 74

[48] J. Goeders, T. Gaskin, and B. Hutchings, "Demand Driven Assembly of FPGA Configurations Using Partial Reconfiguration, Ubuntu Linux, and PYNQ," in 2018 IEEE 26th Annual International Symposium on Field-Programmable Custom Computing Machines (FCCM), April 2018, pp. 149-156. 88

[49] Snyder, Wilson. Verilator. [Online]. Available: https://www.veripool.org/ 90 